\title{
The complete HEFT Lagrangian after the LHC Run I
}

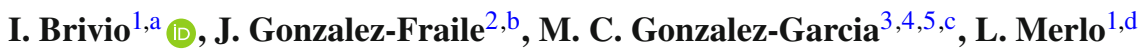 \\ ${ }^{1}$ Departamento de Física Teórica and Instituto de Física Teórica, IFT-UAM/CSIC, Universidad Autónoma de Madrid, Cantoblanco, \\ 28049 Madrid, Spain \\ 2 Institut für Theoretische Physik, Universität Heidelberg, Heidelberg, Germany \\ ${ }^{3}$ C.N. Yang Institute for Theoretical Physics and Department of Physics and Astronomy, SUNY at Stony Brook, Stony Brook, NY 11794-3840, \\ USA \\ ${ }^{4}$ Departament d'Estructura i Constituents de la Matèria and ICC-UB, Universitat de Barcelona, 647 Diagonal, 08028 Barcelona, Spain \\ ${ }^{5}$ Institució Catalana de Recerca y Estudis Avançats (ICREA), Passeig de Lluís Companys 23, 08010 Barcelona, Spain
}

Received: 4 May 2016 / Accepted: 14 June 2016 / Published online: 23 July 2016

(C) The Author(s) 2016. This article is published with open access at Springerlink.com

\begin{abstract}
The complete effective chiral Lagrangian for a dynamical Higgs is presented and constrained by means of a global analysis including electroweak precision data together with Higgs and triple gauge-boson coupling data from the LHC Run I. The operators' basis up to next-to-leading order in the expansion consists of 148 (188 considering righthanded neutrinos) flavour universal terms and it is presented here making explicit the custodial nature of the operators. This effective Lagrangian provides the most general description of the physical Higgs couplings once the electroweak symmetry is assumed, and it allows for deviations from the $S U(2)_{L}$ doublet nature of the Standard Model Higgs. The comparison with the effective linear Lagrangian constructed with an exact $S U(2)_{L}$ doublet Higgs and considering operators with at most canonical dimension six is presented. A promising strategy to disentangle the two descriptions consists in analysing (i) anomalous signals present only in the chiral Lagrangian and not expected in the linear one, that are potentially relevant for LHC searches, and (ii) decorrelation effects between observables that are predicted to be correlated in the linear case and not in the chiral one. The global analysis presented here, which includes several kinematic distributions, is crucial for reducing the allowed parameter space and for controlling the correlations between parameters. This improves previous studies aimed at investigating the Higgs Nature and the origin of the electroweak symmetry breaking.
\end{abstract}

\footnotetext{
a e-mail: ilaria.brivio@uam.es

be-mail: fraile@thphys.uni-heidelberg.de

ce-mail: concha@insti.physics.sunysb.edu

de-mail: luca.merlo@uam.es
}

\section{Contents}

1 Introduction . . . . . . . . . . . . 2

2 The complete HEFT Lagrangian . . . . . . . . . . . 3

2.1 The NLO Lagrangian . . . . . . . . . . . . . . 5

2.2 NLO basis: bosonic sector $\Delta \mathscr{L}_{\text {bos }} \ldots \ldots \ldots 6$

2.2.1 CP even bosonic basis $\Delta \mathscr{L}_{\text {bos }}^{C P} \ldots \ldots .6$

2.2.2 CP odd bosonic basis $\Delta \mathscr{L}_{\mathrm{bos}}^{\mathscr{C P}} \ldots \ldots .7$

2.3 NLO basis: fermionic sector $\Delta \mathscr{L}_{\text {fer }} \ldots \ldots \ldots$

2.3.1 Single fermionic current $\Delta \mathscr{L}_{2 F} \ldots \ldots .8$

Quark current operators . . . . . . . . . 8

Leptonic current operators . . . . . . . . . 9

2.3.2 Four-fermion operators $\Delta \mathscr{L}_{4 F} \ldots \ldots \ldots$

Pure quark operators . . . . . . . . . . 10

Pure leptonic operators . . . . . . . . . . 10

Mixed quark-lepton operators . . . . . . . . 10

2.4 Comparison with the SMEFT basis . . . . . . . 11

3 Phenomenology . . . . . . . . . . . . 12

3.1 Physical parameters definitions . . . . . . . . 12

3.2 Constraints from EWPD . . . . . . . . . . 13

3.3 Effects in Higgs physics . . . . . . . . . . 16

3.4 Triple gauge-boson couplings and Higgs interplay 18

4 Higher order operators and expansion validity . . . . 21

5 Conclusions ................ . . 23

A Additional operators in the presence of RH neutrinos 24

B Removal of $\mathcal{F}(\mathbf{h}) \mathrm{F}(\mathrm{h})$ from the Higgs and fermions kinetic terms . . . . . . . . . . . . . 25

B.1 A practical example . . . . . . . . . . 25

C Construction of the fermionic basis . . . . . . 26

C.1 Useful identities . . . . . . . . . . . . . 26

C.2 Construction of $\Delta \mathscr{L}_{2 F} \ldots \ldots \ldots \ldots$

C.3 Construction of $\Delta \mathscr{L}_{4 F} \ldots \ldots \ldots \ldots$

Four-quark (lepton) operators . . . . . . . . . 27

Mixed quark-lepton operators . . . . . . . . 28 
D Application of the EOMs . . . . . . . . . . . . 28

D.1 Operators that have been removed via EOM . . 29

Bosonic sector . . . . . . . . . . . . . . . . 29

Fermionic sector . . . . . . . . . . . . . . . 29

E Feynman rules . . . . . . . . . . . . . 30

FR: propagators . . . . . . . . . 31

FR: Bosonic . . . . . . . . . . . . 31

FR: Fermionic . . . . . . . . . . . . 35

Single quark current . . . . . . . . . . . . . 35

Single lepton current . . . . . . . . . . . . . 39

Four quarks . . . . . . . . . . . . . 41

Four leptons . . . . . . . . . . . . . 42

Two quark-two leptons . . . . . . . . . . . . 43

References . . . . . . . . . . . . . . . . . . . 44

\section{Introduction}

The discovery of a resonance at LHC [1,2] compatible with the Standard Model (SM) scalar boson ("Higgs" for short) [3-5] opened a new era in particle physics. Now, the on going LHC measurements of the Higgs properties are a crucial step to understand the nature of the Higgs boson and of the Electroweak (EW) symmetry breaking (EWSB).

Without entering into details of specific scenarios, the formalism of Effective Field Theories (EFT) represents an optimal tool for studying the phenomenology of the Higgs sector. In particular, an appropriate description of scenarios in which the Higgs belongs to an elementary $S U(2)$ doublet is provided by the Standard Model EFT (SMEFT). This consists of operators constructed with the SM spectrum, invariant under the Lorentz and SM gauge symmetries and respecting an expansion in canonical mass dimensions $d$. Assuming lepton and baryon number conservation, the first corrections to the SM are provided by operators of dimension six [6,7], suppressed by two powers of the cut-off scale $\Lambda$. Weakly coupled theories are the typical underlying scenarios that can be matched to the SMEFT (also referred to as "linear" Lagrangian) at low energy.

Scenarios where the Higgs does not belong to an elementary exact $S U(2)_{L}$ doublet are still allowed within the current experimental accuracy. This is the case, for example, of composite Higgs models [8-12] or dilaton constructions [13,14]. It is then fundamental and necessary to identify observables that allow one to disentangle these different possibilities. When the Higgs is not required to belong to an exact EW doublet, instead, a useful tool is the so-called Higgs EFT (HEFT) (also dubbed "chiral" Lagrangian). The main difference between SMEFT and HEFT resides in the fact that, in the latter formalism, the physical Higgs $h$ and the ensemble of the three EW Goldstone bosons $\vec{\pi}$ are treated as independent objects, rather than being collectively described by the Higgs doublet. In particular, the physical Higgs $h$ is assigned to a singlet representation of the SM gauge groups. The Goldstone bosons' sector has been studied intensely in the past [15-18] in the context of Higgs-less EWSB scenarios. These works were the first to describe the GBs by means of a dimensionless unitary matrix transforming as a bi-doublet of the global symmetry $S U(2)_{L} \times S U(2)_{R}$,

$\mathbf{U}(x) \equiv e^{i \sigma_{a} \pi^{a}(x) / f_{\pi}}, \quad \mathbf{U}(x) \rightarrow L \mathbf{U}(x) R^{\dagger}$,

being $f_{\pi}$ the scale associated to the SM GBs, and $L, R$ the $S U(2)_{L, R}$ transformations. After EWSB, the invariance under the group $S U(2)_{L} \times S U(2)_{R}$ is broken down to the diagonal $S U(2)_{C}$, commonly called custodial symmetry, and explicitly broken by the gauging of the hypercharge $U(1)_{Y}$ and by the fermion mass splittings. It is customary to introduce two objects, the vector and scalar chiral fields, that transform in the adjoint of $S U(2)_{L}$. They are defined, respectively, as

$\mathbf{V}_{\mu} \equiv\left(\mathbf{D}_{\mu} \mathbf{U}\right) \mathbf{U}^{\dagger}, \quad \mathbf{T} \equiv \mathbf{U} \sigma_{3} \mathbf{U}^{\dagger}$,

where the covariant derivative is given by

$\mathbf{D}_{\mu} \mathbf{U}(x) \equiv \partial_{\mu} \mathbf{U}(x)+i g W_{\mu}(x) \mathbf{U}(x)-\frac{i g^{\prime}}{2} B_{\mu}(x) \mathbf{U}(x) \sigma_{3}$.

Unlike $\mathbf{V}_{\mu}$, $\mathbf{T}$ is not invariant under $S U(2)_{C}$ and can therefore be considered a custodial symmetry breaking spurion. The bosonic Higgs-less EW chiral Lagrangian can then be constructed with $\mathbf{V}_{\mu}$, $\mathbf{T}$ and the gauge-boson field strengths as building blocks, and the tower of invariant operators shall be organised according to a chiral (derivative) expansion [19].

In the last decade, the EW chiral Lagrangian has been extended with the introduction of a light physical Higgs $h$ [20-28], treated as an isosinglet of the SM gauge symmetries. The dependence on the $h$ field is customarily encoded in generic functions $\mathcal{F}(h)$, that are used as building blocks for the construction of the effective operators. These functions are made adimensional by implicitly weighting the insertions of the Higgs field with an opportune suppression scale $f_{h}$, so that one may rewrite the dependence as $\mathcal{F}\left(h / f_{h}\right)$. It is worth underlining that the dependence on the structure $(1+$ $h / v$ ), where $v$ is the EW vacuum expectation value (vev), that characterises the SMEFT Lagrangian is lost in the HEFT and substituted by a generic $h / f_{h}$ expansion.

The typical underlying scenarios that can be described at low energy in terms of the matrix $\mathbf{U}(x)$, the Higgs functions $\mathcal{F}(h)$ and the rest of the SM fields, are those of composite Higgs models [8-12,29]. These assume the existence of some strong ("ultracolour") interaction at high energy, and initially invariant under some global symmetry group $\mathcal{G}$. At the scale $\Lambda_{s}$, the formation of ultracolour condensates breaks spontaneously this invariance, leaving a residual symmetry $\mathcal{H}$ that can embed the EW group. This triggers the appearance of a certain number of Goldstone bosons, among which 
three can be identified with the would-be GBs of the EW group and a fourth one with the Higgs. In such scenarios, all the SM scalars are naturally associated to the same scale $f_{\pi}=f_{h} \equiv f$, with $\Lambda_{s} \leq 4 \pi f$. Spontaneous EWSB is triggered by some explicit breaking of the $\mathcal{H}$ symmetry (provided either by external symmetries [8] or by gauging the SM symmetry together with fermion interactions [11]) and takes place in a second stage. At this level, the Higgs field acquires a vev $\langle h\rangle$, which does not need to coincide with the EW scale $v$, defined by the EW gauge-boson mass: the three quantities $v, f$ and $\langle h\rangle$ are instead related by a model-dependent function. The splitting between $v$ and $f$ constitutes the wellknown fine-tuning of composite Higgs models. It is usually expressed in terms of the parameter by

$\xi \equiv \frac{v^{2}}{f^{2}}$,

that substantially quantifies the degree of non-linearity of the Higgs dynamics. The low-energy projection of composite Higgs models can be described by the HEFT Lagrangian $[30,31]$ and the matching conditions allow one to write the low-energy effective operator coefficients in terms of the high-energy parameters, and the generic functions $\mathcal{F}(h)$ as trigonometric functions of $h / f$. The HEFT Lagrangian can also be used to describe the SMEFT [22-25,30-32], after identifying the operator coefficients of the effective Lagrangians and writing all the $\mathcal{F}(h)$ functions in terms of $(1+h / v)$. Dilaton constructions [13,14] or even more exotic models, where the Higgs is an EW singlet, can also be described by the HEFT Lagrangian.

Without assuming any specific underlying scenario or comparing with SMEFT, the $v / f_{h}$ and $v / f_{\pi}$ parameters are not physical and can be reabsorbed in the operators coefficients and in the coefficients of the $\mathcal{F}(h)$ functions. This is tantamount to substituting $f_{\pi}$ and $f_{h}$ by $v$, which ensures canonical kinetic terms for the GBs and fixes the correct order of magnitude for the gauge bosons masses, without fine-tunings. This notation will be employed in the following, unless otherwise specified.

The disparities between the SMEFT and the HEFT originate from the different nature of the building blocks used in the construction of the effective operators. The independence between the GB field $\mathbf{U}(x)$ and the physical $h$, together with the fact that $h$ does not transform under the SM gauge symmetries, leads to a different ordering of the chiral effective operators compared to the linear ones. As a result, at any given order in the expansion the number of chiral independent operators is much larger than in the SMEFT case. The corresponding phenomenology, focussing on the bosonic part of the Lagrangian, has been studied in Refs. [24,25], where signatures that may allow one to discriminate between an elementary and a dynamical Higgs have also been identified. These signatures include sets of couplings that are pre- dicted to be correlated in an elementary Higgs scenario but are generically decorrelated in the dynamical case, as well as effects that are expected to be suppressed in the linear realisation but may appear at the lowest order in the chiral expansion. These signatures are also typical in Dark Matter studies when the Higgs is not taken to be an exact $S U(2)_{L}$ doublet [33]. Complementary signatures that can distinguish between SMEFT and HEFT also include the scattering of the longitudinal components of the gauge bosons [34-36].

The complete non-redundant HEFT Lagrangian including both bosonic and fermionic operators has been constructed in this work and is presented in Sect. 2, making explicit the custodial nature of the operators. The HEFT basis is formed by 148 independent flavour universal operators altogether, whose extension to generic flavour contractions is straightforward. The Lagrangian does not account for the presence of right-handed neutrinos, whose inclusion in the spectrum would imply the addition 40 extra operators to the basis, listed in Appendix A. Section 2 also contains a comparison between the HEFT Lagrangian and the SMEFT one, while a phenomenological analysis of the HEFT basis is presented in Sect. 3. The study considers all the available collider data, which includes electroweak precision measurements and Higgs and triple gauge-boson vertex (TGV) data from the LHC Run I. To the best of our knowledge, this is the first time that such analysis has been done for the complete HEFT description. Finally, Sect. 4, contains a discussion of the impact of higher order operators: a set of invariants that may become relevant at the increased energies foreseen for the LHC and future colliders is also pointed out. The conclusions are presented in Sect. 5, while some more technical details are deferred to the appendices, together with the Feynman Rules for the CP even subset of HEFT operators.

\section{The complete HEFT Lagrangian}

In this section we review the construction of the HEFT Lagrangian, in a notation similar to that of Refs. [22$25,32,37]$. The bosonic building blocks are the gauge field strengths $B_{\mu \nu}, W_{\mu \nu}, \mathcal{G}_{\mu \nu}$, the vector and scalar chiral fields $\mathbf{V}_{\mu}$ and $\mathbf{T}$ defined in Eq. (1.2) and the functions $\mathcal{F}(h)$ introduced in the previous section. The SM fermions are conveniently grouped into doublets of the global $S U(2)_{L, R}$ symmetries:

$$
\begin{aligned}
Q_{L} & =\left(\begin{array}{c}
U_{L} \\
D_{L}
\end{array}\right), \quad Q_{R}=\left(\begin{array}{c}
U_{R} \\
D_{R}
\end{array}\right), \quad L_{L}=\left(\begin{array}{c}
v_{L} \\
E_{L}
\end{array}\right), \\
L_{R} & =\left(\begin{array}{c}
0 \\
E_{R}
\end{array}\right) .
\end{aligned}
$$

This choice allows one to have a more compact notation for the fermionic operators. The $S U(2)_{R}$ doublet structure can easily be broken with the insertion of the custodial symmetry 
breaking spurion $\mathbf{T}$. Notice that the $L_{R}$ doublet only includes right-handed charged leptons. The inclusion of right-handed neutrinos requires an extension of the fermionic basis presented in Sect. 2.3 with the addition of the operators listed in Appendix A.

The HEFT Lagrangian can be written as a sum of two terms,

$\mathscr{L}_{\mathrm{HEFT}} \equiv \mathscr{L}_{0}+\Delta \mathscr{L}$

where the first term contains the leading order (LO) operators and the second one accounts for new interactions and for deviations from the LO.

The LO Lagrangian includes the kinetic terms for all the particles in the spectrum, the Yukawa couplings and the scalar potential $^{1}$ :

$$
\begin{aligned}
\mathscr{L}_{0}= & -\frac{1}{4} \mathcal{G}_{\mu \nu}^{\alpha} \mathcal{G}^{\alpha \mu \nu}-\frac{1}{4} W_{\mu \nu}^{a} W^{a \mu \nu}-\frac{1}{4} B_{\mu \nu} B^{\mu \nu} \\
& +\frac{1}{2} \partial_{\mu} h \partial^{\mu} h-\frac{v^{2}}{4} \operatorname{Tr}\left(\mathbf{V}_{\mu} \mathbf{V}^{\mu}\right) \mathcal{F}_{C}(h)-V(h) \\
& +i \bar{Q}_{L} \not D Q_{L}+i \bar{Q}_{R} \not D Q_{R}+i \bar{L}_{L} \not D L_{L}+i \bar{L}_{R} \not D L_{R} \\
& -\frac{v}{\sqrt{2}}\left(\bar{Q}_{L} \mathbf{U} \mathcal{Y}_{Q}(h) Q_{R}+\text { h.c. }\right) \\
& -\frac{v}{\sqrt{2}}\left(\bar{L}_{L} \mathbf{U} \mathcal{Y}_{L}(h) L_{R}+\text { h.c. }\right) \\
& -\frac{g_{s}^{2}}{16 \pi^{2}} \lambda_{s} \mathcal{G}_{\mu \nu}^{\alpha} \tilde{\mathcal{G}}^{\alpha \mu \nu},
\end{aligned}
$$

where $\mathcal{G}^{\mu \nu} \equiv \frac{1}{2} \epsilon^{\mu \nu \rho \sigma} \mathcal{G}_{\rho \sigma}$. The first line describes the kinetic terms of the gauge bosons; the second line contains the Higgs and Goldstone bosons' kinetic term, the scalar potential, and the mass terms for the EW gauge bosons; the third line presents the kinetic terms for all the fermions, while the fourth line accounts for the Yukawa interactions. Finally, the last line contains the theta term of QCD. The function $\mathcal{F}_{C}(h)$ appearing in the kinetic term for the GBs can be expanded as

$\mathcal{F}_{C}(h)=1+2 a_{C} \frac{h}{v}+b_{C} \frac{h^{2}}{v^{2}}+\cdots$

where the dots account for higher powers of $(h / v)$. For the phenomenological analysis it is convenient to single out the BSM part of the coefficients $a_{C}, b_{C}$, using the notation

$a_{C}=1+\Delta a_{C}, \quad b_{C}=1+\Delta b_{C}$,

where $\Delta a_{C}, \Delta b_{C}$ will be assumed to be of the same order as the coefficients accompanying the operators appearing in $\Delta \mathscr{L}$. The functions $\mathcal{Y}_{Q, L}(h)$ appearing in the Yukawa cou-

\footnotetext{
${ }^{1}$ Comments on the construction of the LO Lagrangian in Eq. (2.3) are given in Appendix B.
}

plings have an analogous structure to $\mathcal{F}_{C}(h)$ :

$$
\begin{aligned}
& \mathcal{Y}_{Q}(h) \equiv \operatorname{diag}\left(\sum_{n} Y_{U}^{(n)} \frac{h^{n}}{v^{n}}, \sum_{n} Y_{D}^{(n)} \frac{h^{n}}{v^{n}}\right), \\
& \mathcal{Y}_{L}(h) \equiv \operatorname{diag}\left(0, \sum_{n} Y_{\ell}^{(n)} \frac{h^{n}}{v^{n}}\right) .
\end{aligned}
$$

The $n=0$ terms yield fermion masses, while the higher orders describe the interaction with $n$ insertions of the Higgs field $h$, accounting in general for non-aligned contributions.

The kinetic terms of the fermions and of the physical Higgs are not accompanied by any $\mathcal{F}(h)$ since, as shown in Appendix B, it is always possible to reabsorb their contributions inside the generic functions $\mathcal{F}_{C}(h)$ and $\mathcal{Y}_{Q, L}(h)$. This can be done either via a field redefinition or, alternatively, applying the Equations of Motion (EOMs) (the two procedures are not equivalent in general, but lead to the same result at first order in the deviations from the LO). Moreover, the kinetic terms of the gauge bosons in the first line of Eq. (2.3) do not come associated with any $\mathcal{F}(h)$, assuming that the transverse components of the gauge fields, described by the gauge field strength, do not couple strongly to the Higgs sector. These couplings can be neglected at the LO and be considered, instead, at the next-to-leading order (NLO).

$\Delta \mathscr{L}$ contains higher order operators with respect to those appearing in $\mathscr{L}_{0}$. The precise ordering of these operators depends on the choice of a specific power counting rule. The HEFT can be seen as a fusion of two theories, the chiral perturbation approach associated to the SM GBs-i.e. the longitudinal components of the gauge bosons-and the traditional linear description that applies to the transverse components of the gauge bosons and to fermions. The physical $h$ should also undergo the chiral perturbation description as it enters in the Lagrangian via the adimensional functions $\mathcal{F}(h)$ : the latter can be interpreted as playing the same role as the adimensional GB matrix field $\mathbf{U}(x)$. Indeed, in concrete composite Higgs models, the pseudo-GB nature of the Higgs forces the $\mathcal{F}(h)$ functions to take trigonometric structures [30]. Being the HEFT a merging between linear and chiral descriptions, the counting rules which apply singularly to each of the expansions hold simultaneously for the HEFT [38]. As a result, the LO Lagrangian in Eq. (2.3) itself does not strictly respect the chiral expansion: $\mathscr{L}_{0}$ contains both operators with two derivatives and the gauge-boson kinetic terms, which has four derivatives; at the same time, some two-derivative operators have been excluded from the LO. On the other hand, $\mathscr{L}_{0}$ does not even follow an expansion in canonical dimensions, as for instance the Yukawa interactions and the gauge-boson mass term present an infinite series of $h$ legs, contrary to all the other terms in the LO Lagrangian. 
The renormalisability conditions are also different in the two descriptions. In the linear expansion an $n$-loop diagram containing one single $d=6$ vertex generates divergent contributions that can be reabsorbed by other $d=6$ operators and do not require the introduction of any higher-dimensional operator. On the contrary, in the chiral case, 1-loop diagrams with $n$ insertions of a two-derivative coupling, usually listed in the LO Lagrangian, produce divergences that require the introduction of operators with four-derivatives, which generically constitute the NLO Lagrangian.

Finally, the HEFT presents an additional aspect that makes it hard to identify a proper counting rule: the presence of multiple scales. Besides the cut-off of the theory $\Lambda$, one should consider the presence of the GB scale $f_{\pi}$ and of the $h$-scale $f_{h}$. Although it may happen that the last two coincide with $f_{\pi}=f_{h}=f$ and that they are related to the first one by the constraint $\Lambda \leq 4 \pi f$ (which is the case in composite Higgs models), the three scales are in principle independent and associated to different physical quantities. On top of this, one should not forget the fine-tuning associated to the EW scale $v$ and parametrised by $\xi$ defined in Eq. (1.4). In practice, the counting rule associated to the HEFT depends on more than one expansion parameters and may vary depending on the typical energy scale of the observables considered in the phenomenological analysis.

In conclusion, rather than basing the choice of the NLO Lagrangian operators on a sophisticated counting rule whose applicability is not valid in full generality, here the selection is performed with the following strategy. An NLO operator should satisfy at least one of the criteria below:

- It is necessary for reabsorbing 1-loop divergences arising from the renormalisation of $\mathscr{L}_{0}$.

- It presents the same suppression as the operators in the first class and receives finite 1-loop contributions: for instance, all the four-fermion operators are included in the NLO, in spite of the fact that only a subset of these is required to reabsorb 1-loop divergences.

- It has been left out from the LO Lagrangian due to phenomenological reasons.

The suppression factor of each operator is determined using the NDA master formula, first proposed in Ref. [39] and later modified in Refs. [38,40]. Following the notation of Ref. [38]:

$$
\begin{aligned}
\frac{\Lambda^{4}}{16 \pi^{2}} & {\left[\frac{\partial}{\Lambda}\right]^{N_{p}}\left[\frac{4 \pi \phi}{\Lambda}\right]^{N_{\phi}}\left[\frac{4 \pi A}{\Lambda}\right]^{N_{A}} } \\
\times & {\left[\frac{4 \pi \psi}{\Lambda^{3 / 2}}\right]^{N_{\psi}}\left[\frac{g}{4 \pi}\right]^{N_{g}}\left[\frac{y}{4 \pi}\right]^{N_{y}}, }
\end{aligned}
$$

where $\phi$ represents either the SM GBs or $h, \psi$ a generic fermion, $A$ a generic gauge field, $g$ the gauge couplings and $y$ the Yukawa couplings. All the operators appearing in the LO Lagrangian in Eq. (2.3) are normalised according to this formula, apart from the operators providing gauge-boson masses, $\left(v^{2} / 4\right) \operatorname{Tr}\left(\mathbf{V}_{\mu} \mathbf{V}^{\mu}\right) \mathcal{F}_{C}(h)$, and fermions' masses $(v \sqrt{2}) \bar{\psi}_{L} \mathbf{U} \mathcal{Y}_{\psi}(h) \psi_{R}$, which are multiplied by powers of the EW scale $v$ and not by $\Lambda$ or $f$ as expected. This is due to the well-known fine-tuning, typical of theories where the EWSB sector is non-linearly realised. Notice that with these conventions all the kinetic terms are canonically normalised, differently from the following using the original version of the NDA master formula from Ref. [39].

The master formula also ensures that the operators belonging to the NLO Lagrangian are typically suppressed with respect to those of $\mathscr{L}_{0}$ by powers of $(4 \pi)^{(n \leq 2)}$, reflecting the renormalisation of the chiral sector, and/or by powers of $\Lambda^{(n \leq 2)}$, associated to possible new physics contributions. Different cases will be discussed when necessary.

\subsection{The NLO Lagrangian}

The second part of the HEFT Lagrangian, $\Delta \mathscr{L}$, contains in general all the invariant operators appearing beyond the leading order. They include corrections to the interactions contained in $\mathscr{L}_{0}$ as well as completely new couplings. This Lagrangian can be generically written as a sum of two parts

$\Delta \mathscr{L}=\Delta \mathscr{L}_{\text {bos }}+\Delta \mathscr{L}_{\text {fer }}$,

where $\Delta \mathscr{L}_{\text {bos }}$ contains all the purely bosonic operators, while $\Delta \mathscr{L}_{\text {fer }}$ accounts for the interactions that involve fermions.

In this work, $\Delta \mathscr{L}$ will be restricted to the NLO, defined according to the rules presented in the previous section. An alternative construction of a NLO Lagrangian was derived in Ref. [26]. We present a set of invariants that forms a complete, non-redundant basis at this order in the effective expansion, which has been constructed identifying first a complete basis for each of the two sectors individually (bosonic and fermionic) and subsequently employing the EOMs to remove redundant terms.

Given the large number of invariants, the operators are classified as follows: the bosonic basis is split into CP conserving and $\mathrm{CP}$ violating subsets (the field $h$ is assumed to be a CP even scalar):

$\Delta \mathscr{L}_{\mathrm{bos}}=\Delta \mathscr{L}_{\mathrm{bos}}^{C P}+\Delta \mathscr{L}_{\mathrm{bos}}^{C P}$

while in the fermionic sector the distinction is between fermionic single- and double-current structures:

$\Delta \mathscr{L}_{\text {fer }}=\Delta \mathscr{L}_{2 F}+\Delta \mathscr{L}_{4 F}$

The operators are named differently according to the category to which they belong and each of them includes a function $\mathcal{F}_{i}(h)$ conventionally parametrised as 
$\mathcal{F}_{i}(h)=1+2 a_{i} \frac{h}{v}+b_{i} \frac{h^{2}}{v^{2}}+\cdots$

Moreover, each effective operator is multiplied with a real coefficient, indicated with a lowercase letter $(c, \tilde{c}, n, r)$ associated to each class. The following table defines the notation and summarises the number of independent invariants for each set, in the absence of right-handed neutrinos and after the application of the EOMs.

\begin{tabular}{lllr}
\hline $\mathscr{L}$ & Sub-category & Notation & \# operators \\
\hline$\Delta \mathscr{L}_{\text {bos }}^{C P}$ & & $c_{j} \mathcal{P}_{j}$ & 26 \\
$\Delta \mathscr{L}_{\text {bos }}$ & & $\tilde{c}_{j} \mathcal{S}_{j}$ & 16 \\
$\Delta \mathscr{L}_{2 F}$ & Quark current & $n_{j}^{\mathcal{Q}} \mathcal{N}_{j}^{\mathcal{Q}}$ & 36 \\
& Lepton current & $n_{j}^{\ell} \mathcal{N}_{j}^{\ell}$ & 14 \\
$\Delta \mathscr{L}_{4 F}$ & Four quarks & $r_{j}^{\mathcal{Q}} R_{j}^{\mathcal{Q}}$ & 26 \\
& Four leptons & $r_{j}^{\ell} R_{j}^{\ell}$ & 7 \\
& Two quarks and two leptons & $r_{j}^{\mathcal{Q} \ell} R_{j}^{\mathcal{Q} \ell}$ & 23 \\
Tot & & & 148 \\
\hline
\end{tabular}

Forty additional operators should be considered if righthanded neutrinos are added to the spectrum: 17 in $\mathscr{L}_{2 F}$, eight four-lepton interactions and 15 mixed two-quark-two-lepton terms.

The complete list of NLO operators is provided in the following: Sects. 2.2 and 2.3 are, respectively, dedicated to the bosonic and fermionic sectors. Further details of the construction of the invariants and of how the EOMs have been employed to remove redundant terms can be found in Appendices $\mathrm{C}$ and $\mathrm{D}$. The Feynman rules of the complete $\mathrm{CP}$ conserving basis are reported in Appendix E, in unitary gauge and for vertices with up to four legs.

\subsection{NLO basis: bosonic sector $\Delta \mathscr{L}_{\text {bos }}$}

At NLO in the chiral expansion, the Lagrangian $\Delta \mathscr{L}_{\text {bos }}$ contains purely bosonic operators. Complete bases for the $\mathrm{CP}$ even and CP odd sectors have been already constructed in Refs. [22,24,25] respectively. In this work only a subset of those ensembles are retained as, once the fermionic sector is introduced, some of the terms become redundant and can be removed using the EOMs (see Appendix D). Nonetheless, the original numeration of the operators has been kept, in order to simplify the comparison with the literature. Finally, the explicit formal dependence on $h$ in the generic functions $\mathcal{F}_{i}(h)$ is dropped in the following for brevity.

\subsubsection{CP even bosonic basis $\Delta \mathscr{L}_{\text {bos }}^{C P}$}

The CP even NLO Lagrangian reads

$$
\Delta \mathscr{L}_{\mathrm{bos}}^{C P}=\sum_{j} c_{j} \mathcal{P}_{j}(h),
$$

with

$$
j=\{T, B, W, G, D H, 1-6,8,11-14,17,18,20-24,
$$

$$
\text { 26, } W W W, G G G\}
$$

where all the operators contain four derivatives, with the exception of

$\mathcal{P}_{T}(h)=\frac{v^{2}}{4} \operatorname{Tr}\left(\mathbf{T V} \mathbf{V}_{\mu}\right) \operatorname{Tr}\left(\mathbf{T} \mathbf{V}^{\mu}\right) \mathcal{F}_{T}$

and

$$
\begin{aligned}
& \mathcal{P}_{W W W}(h)=\frac{4 \pi \varepsilon_{a b c}}{\Lambda^{2}} W_{\mu}^{a v} W_{\nu}^{b \rho} W_{\rho}^{c \mu} \mathcal{F}_{W W W}, \\
& \mathcal{P}_{G G G}(h)=\frac{4 \pi f_{\alpha \beta \gamma}}{\Lambda^{2}} G_{\mu}^{\alpha \nu} G_{\nu}^{\beta \rho} G_{\rho}^{\gamma \mu} \mathcal{F}_{G G G},
\end{aligned}
$$

where $f_{\alpha \beta \gamma}$ denotes the structure constants of $S U$ (3).

The two-derivative operator $\mathcal{P}_{T}(h)$ is very similar to $v^{2} \operatorname{Tr}\left(\mathbf{V}_{\mu} \mathbf{V}^{\mu}\right) \mathcal{F}_{C}$ and, therefore, it could have been included in $\mathscr{L}_{0}$ a priori. However, it is customary to move it to $\Delta \mathscr{L}$ because the bounds existing on its coefficient are quite strong: $c_{T} \lesssim 10^{-2}$. In fact, this operator violates the custodial symmetry and contributes to the $T$ parameter, which is constrained to a high accuracy by electroweak precision data (EWPD). In order to avoid irrelevant contributions to the EOMs, this operator has been moved to the NLO, which is justifiable assuming an approximately preserved custodial symmetry. ${ }^{2}$ The two operators $\mathcal{P}_{W W W}(h)$ and $\mathcal{P}_{G G G}(h)$ are not required to absorb divergences due to the 1-loop renormalisation. However, they can be listed among the NLO operators: containing only the transverse components of the gauge bosons, they follow the linear description; then they come suppressed by $\Lambda^{2}$, on the same foot as the four-fermion operators. It will be shown in the following that they have a non-trivial impact at the phenomenological level.

The remaining 23 operators in $\Delta \mathscr{L}_{\text {bos }}^{C P}$, in the numeration of Ref. [24], are the following:

$$
\begin{aligned}
& \mathcal{P}_{B}(h)=-\frac{1}{4} B_{\mu \nu} B^{\mu \nu} \mathcal{F}_{B}, \\
& \mathcal{P}_{W}(h)=-\frac{1}{4} W_{\mu \nu}^{a} W^{a \mu \nu} \mathcal{F}_{W}, \\
& \mathcal{P}_{G}(h)=-\frac{1}{4} G_{\mu \nu}^{a} G^{a \mu \nu} \mathcal{F}_{G}, \\
& \mathcal{P}_{D H}(h)=\left(\partial_{\mu} \mathcal{F}_{D H}(h) \partial^{\mu} \mathcal{F}_{D H}^{\prime}(h)\right)^{2}, \\
& \mathcal{P}_{1}(h)=B_{\mu \nu} \operatorname{Tr}\left(\mathbf{T} W^{\mu \nu}\right) \mathcal{F}_{1}, \\
& \mathcal{P}_{2}(h)=\frac{i}{4 \pi} B_{\mu \nu} \operatorname{Tr}\left(\mathbf{T}\left[\mathbf{V}^{\mu}, \mathbf{V}^{\nu}\right]\right) \mathcal{F}_{2},
\end{aligned}
$$

\footnotetext{
$\overline{2 \text { Although the }} T$ parameter only constrains the $h$-independent coupling of $\mathcal{P}_{T}(h)$, the whole operator has been moved to the NLO Lagrangian. This follows the basic assumption that for a given operator the $h^{n>0}$ coefficients are of the same order as the $h^{0}$ coefficient. Indeed, if an operator is suppressed due to a symmetry principle, this applies to any of the $h^{n \geq 0}$ couplings.
} 


$$
\begin{aligned}
& \mathcal{P}_{3}(h)=\frac{i}{4 \pi} \operatorname{Tr}\left(W_{\mu \nu}\left[\mathbf{V}^{\mu}, \mathbf{V}^{\nu}\right]\right) \mathcal{F}_{3}, \\
& \mathcal{P}_{4}(h)=\frac{i}{4 \pi} B_{\mu \nu} \operatorname{Tr}\left(\mathbf{T V}^{\mu}\right) \partial^{\nu} \mathcal{F}_{4}, \\
& \mathcal{P}_{5}(h)=\frac{i}{4 \pi} \operatorname{Tr}\left(W_{\mu \nu} \mathbf{V}^{\mu}\right) \partial^{\nu} \mathcal{F}_{5}, \\
& \mathcal{P}_{6}(h)=\frac{1}{(4 \pi)^{2}}\left(\operatorname{Tr}\left(\mathbf{V}_{\mu} \mathbf{V}^{\mu}\right)\right)^{2} \mathcal{F}_{6}, \\
& \mathcal{P}_{8}(h)=\frac{1}{(4 \pi)^{2}} \operatorname{Tr}\left(\mathbf{V}_{\mu} \mathbf{V}_{v}\right) \partial^{\mu} \mathcal{F}_{8} \partial^{\nu} \mathcal{F}_{8}^{\prime}, \\
& \mathcal{P}_{11}(h)=\frac{1}{(4 \pi)^{2}}\left(\operatorname{Tr}\left(\mathbf{V}_{\mu} \mathbf{V}_{\nu}\right)\right)^{2} \mathcal{F}_{11}, \\
& \mathcal{P}_{12}(h)=\left(\operatorname{Tr}\left(\mathbf{T} W_{\mu \nu}\right)\right)^{2} \mathcal{F}_{12}, \\
& \mathcal{P}_{13}(h)=\frac{i}{4 \pi} \operatorname{Tr}\left(\mathbf{T} W_{\mu \nu}\right) \operatorname{Tr}\left(\mathbf{T}\left[\mathbf{V}^{\mu}, \mathbf{V}^{\nu}\right]\right) \mathcal{F}_{13}, \\
& \mathcal{P}_{14}(h)=\frac{\varepsilon^{\mu \nu \rho \lambda}}{4 \pi} \operatorname{Tr}\left(\mathbf{T} \mathbf{V}_{\mu}\right) \operatorname{Tr}\left(\mathbf{V}_{\nu} W_{\rho \lambda}\right) \mathcal{F}_{14}, \\
& \mathcal{P}_{17}(h)=\frac{i}{4 \pi} \operatorname{Tr}\left(\mathbf{T} W_{\mu \nu}\right) \operatorname{Tr}\left(\mathbf{T} \mathbf{V}^{\mu}\right) \partial^{\nu} \mathcal{F}_{17}, \\
& \mathcal{P}_{18}(h)=\frac{1}{(4 \pi)^{2}} \operatorname{Tr}\left(\mathbf{T}\left[\mathbf{V}_{\mu}, \mathbf{V}_{\nu}\right]\right) \operatorname{Tr}\left(\mathbf{T} \mathbf{V}^{\mu}\right) \partial^{\nu} \mathcal{F}_{18}, \\
& \mathcal{P}_{20}(h)=\frac{1}{(4 \pi)^{2}} \operatorname{Tr}\left(\mathbf{V}_{\mu} \mathbf{V}^{\mu}\right) \partial_{\nu} \mathcal{F}_{20} \partial^{\nu} \mathcal{F}_{20}^{\prime}, \\
& \mathcal{P}_{21}(h)=\frac{1}{(4 \pi)^{2}}\left(\operatorname{Tr}\left(\mathbf{T V} \mathbf{V}_{\mu}\right)\right)^{2} \partial_{\nu} \mathcal{F}_{21} \partial^{\nu} \mathcal{F}_{21}^{\prime} \\
& \mathcal{P}_{22}(h)=\frac{1}{(4 \pi)^{2}} \operatorname{Tr}\left(\mathbf{T} \mathbf{V}_{\mu}\right) \operatorname{Tr}\left(\mathbf{T} \mathbf{V}_{v}\right) \partial^{\mu} \mathcal{F}_{22} \partial^{v} \mathcal{F}_{22}^{\prime}, \\
& \mathcal{P}_{23}(h)=\frac{1}{(4 \pi)^{2}} \operatorname{Tr}\left(\mathbf{V}_{\mu} \mathbf{V}^{\mu}\right)\left(\operatorname{Tr}\left(\mathbf{T} \mathbf{V}_{\nu}\right)\right)^{2} \mathcal{F}_{23}, \\
& \mathcal{P}_{24}(h)=\frac{1}{(4 \pi)^{2}} \operatorname{Tr}\left(\mathbf{V}_{\mu} \mathbf{V}_{\nu}\right) \operatorname{Tr}\left(\mathbf{T} \mathbf{V}^{\mu}\right) \operatorname{Tr}\left(\mathbf{T} \mathbf{V}^{\nu}\right) \mathcal{F}_{24}, \\
& \mathcal{P}_{26}(h)=\frac{1}{(4 \pi)^{2}}\left(\operatorname{Tr}\left(\mathbf{T} \mathbf{V}_{\mu}\right) \operatorname{Tr}\left(\mathbf{T} \mathbf{V}_{\nu}\right)\right)^{2} \mathcal{F}_{26}
\end{aligned}
$$

As anticipated in the previous section, while the kinetic terms for the gauge bosons are listed at the $\mathrm{LO}$, the interactions obtained after introducing the dependence on $h$ are reported in the list of NLO operators, under the assumption that the coupling of the transverse components of the gauge fields with the Higgs sector is a subleading effect.

It is also worth commenting on the operators $\mathcal{P}_{1}(h)$ and $\mathcal{P}_{12}(h)$ : these two structures, including the terms without $h$ insertions, are customarily listed among the NLO terms despite their similarity with the gauge-boson kinetic terms. This is justified, a posteriori, by the fact that they contribute to the $S$ and $U$ parameters, respectively (see Sect. 3.2), which are strongly constrained. In this sense, their treatment is analogous to that of $\mathcal{P}_{T}(h)$.

The operators $\mathcal{P}_{C}(h)$ and $\mathcal{P}_{H}(h)$ of Ref. [24] have not been included in this list, as their effects can be reabsorbed in redefinitions of the arbitrary functions $\mathcal{F}_{C}(h)$ and $\mathcal{Y}_{Q, L}(h)$ appearing in $\mathscr{L}_{0}$ in Eq. (2.3) (see Appendix B). Moreover, compared to Ref. [24], a different normalisation for the operators has been chosen: the $4 \pi$ suppression factors determined by the NDA master formula in Eq. (2.7) have been made explicit (see Ref. [38] for details of the advantages of the NDA normalisation), while the dependence on the coupling constants has been removed, in order to emphasise the generality of the EFT approach. It is customary, indeed, to include in the definition of the HEFT operators the numerical factors arising from the 1-loop renormalisation procedure (see Refs. [41,42] for a general discussion in the SMEFT case): for instance, the operator $\mathcal{P}_{1}(h)$ is often defined proportionally to $g g^{\prime} /(4 \pi)^{2}[17,18,22,24]$. However, in principle the coefficients $c_{i}$ account not only for renormalisation effects, but also for possible external contributions, originating by sources that do not need to share the same dependence on the gauge couplings. This normalisation choice is common in many EFTs, such as Fermi's theory, the EFT for mesons processes and the SMEFT.

\subsubsection{CP odd bosonic basis $\triangle \mathscr{L}_{\text {bos }}^{C P}$}

In the $\mathrm{CP}$ odd sector the bosonic Lagrangian contains 16 operators: according to Ref. [25],

$$
\begin{aligned}
& \Delta \mathscr{L}_{\mathrm{bos}}^{C P}=\sum_{j} \tilde{c}_{j} \mathcal{S}_{j}, \\
& \quad j=\{2 D, \tilde{B}, \tilde{W}, \tilde{G}, 1-9,15, \widetilde{W} W W, \widetilde{G} G G\},
\end{aligned}
$$

where, as for $\Delta \mathscr{L}_{\text {bos }}^{C P}$, all the operators have four derivatives, with the exception of

$$
\mathcal{S}_{2 D}(h) \equiv i \frac{v^{2}}{4} \operatorname{Tr}\left(\mathbf{T} \mathbf{V}_{\mu}\right) \partial^{\mu} \mathcal{F}_{2 D}
$$

and

$$
\begin{aligned}
& \mathcal{S}_{\widetilde{W} W W}(h)=\frac{4 \pi \varepsilon_{a b c}}{\Lambda^{2}} \widetilde{W}_{\mu}^{a v} W_{\nu}^{b \rho} W_{\rho}^{c \mu} \mathcal{F}_{\widetilde{W} W W}, \\
& \mathcal{S}_{\widetilde{G} G G}(h)=\frac{4 \pi f_{\alpha \beta \gamma}}{\Lambda^{2}} \widetilde{G}_{\mu}^{\alpha \nu} G_{\nu}^{\beta \rho} G_{\rho}^{\gamma \mu} \mathcal{F}_{\widetilde{G} G G} .
\end{aligned}
$$

The rest of operators entering $\Delta \mathscr{L}_{\text {bos }}^{C P}$ are

$\mathcal{S}_{\widetilde{B}}(h) \equiv-B^{\mu \nu} \widetilde{B}_{\mu \nu} \mathcal{F}_{\widetilde{B}}$,

$\mathcal{S}_{\widetilde{W}}(h) \equiv-\operatorname{Tr}\left(W^{\mu \nu} \widetilde{W}_{\mu \nu}\right) \mathcal{F}_{\widetilde{W}}$,

$\mathcal{S}_{\widetilde{G}}(h) \equiv-G^{a \mu \nu} \widetilde{G}_{\mu \nu}^{a} \mathcal{F}_{\widetilde{G}}$,

$\mathcal{S}_{1}(h) \equiv \widetilde{B}^{\mu \nu} \operatorname{Tr}\left(\mathbf{T} W_{\mu \nu}\right) \mathcal{F}_{1}$,

$\mathcal{S}_{2}(h) \equiv \frac{i}{4 \pi} \widetilde{B}^{\mu \nu} \operatorname{Tr}\left(\mathbf{T} \mathbf{V}_{\mu}\right) \partial_{\nu} \mathcal{F}_{2}$,

$\mathcal{S}_{3}(h) \equiv \frac{i}{4 \pi} \operatorname{Tr}\left(\tilde{W}^{\mu \nu} \mathbf{V}_{\mu}\right) \partial_{\nu} \mathcal{F}_{3}$,

$\mathcal{S}_{4}(h) \equiv \frac{1}{4 \pi} \operatorname{Tr}\left(W^{\mu \nu} \mathbf{V}_{\mu}\right) \operatorname{Tr}\left(\mathbf{T} \mathbf{V}_{\nu}\right) \mathcal{F}_{4}$,

$\mathcal{S}_{5}(h) \equiv \frac{i}{(4 \pi)^{2}} \operatorname{Tr}\left(\mathbf{V}^{\mu} \mathbf{V}^{\nu}\right) \operatorname{Tr}\left(\mathbf{T} \mathbf{V}_{\mu}\right) \partial_{\nu} \mathcal{F}_{5}$, 


$$
\begin{aligned}
\mathcal{S}_{6}(h) & \equiv \frac{i}{(4 \pi)^{2}} \operatorname{Tr}\left(\mathbf{V}^{\mu} \mathbf{V}_{\mu}\right) \operatorname{Tr}\left(\mathbf{T} \mathbf{V}^{\nu}\right) \partial_{\nu} \mathcal{F}_{6}, \\
\mathcal{S}_{7}(h) & \equiv \frac{1}{4 \pi} \operatorname{Tr}\left(\mathbf{T}\left[W^{\mu \nu}, \mathbf{V}_{\mu}\right]\right) \partial_{\nu} \mathcal{F}_{7}, \\
\mathcal{S}_{8}(h) & \equiv \operatorname{Tr}\left(\mathbf{T} \widetilde{W}^{\mu \nu}\right) \operatorname{Tr}\left(\mathbf{T} W_{\mu \nu}\right) \mathcal{F}_{8}, \\
\mathcal{S}_{9}(h) & \equiv \frac{i}{4 \pi} \operatorname{Tr}\left(\mathbf{T} \tilde{W}^{\mu \nu}\right) \operatorname{Tr}\left(\mathbf{T} \mathbf{V}_{\mu}\right) \partial_{\nu} \mathcal{F}_{9}, \\
\mathcal{S}_{15}(h) & \equiv \frac{i}{(4 \pi)^{2}} \operatorname{Tr}\left(\mathbf{T} \mathbf{V}^{\mu}\right)\left(\operatorname{Tr}\left(\mathbf{T} \mathbf{V}^{\nu}\right)\right)^{2} \partial_{\mu} \mathcal{F}_{15} .
\end{aligned}
$$

As for the CP even part of the bosonic basis, the explicit dependence on the gauge couplings is not part of the definition of the operators, while the $4 \pi$ factors are reported according to Eq. (2.7).

The operator $\mathcal{S}_{2 D}(h)$ deserves a special remark. Being a two-derivative operator, it would be naturally listed at the LO. However, restricting for simplicity the discussion to the unitary gauge, $\mathcal{S}_{2 D}(h)$ introduces a mixing between the gauge boson $Z$ and the physical $h$, that can be rotated away via a proper redefinition of the Goldstone bosons' matrix, as detailed in Refs. [25,43]:

$\mathbf{U} \rightarrow \tilde{\mathbf{U}} \exp \left[-i a_{2 D} \tilde{c}_{2 D} \frac{h}{v} \sigma_{3}\right]$.

At leading order in the effective coefficients, the effects of this operator are eventually recast into $\mathrm{CP}$ odd contributions to the Yukawa couplings with arbitrary number of $h$ legs and to the vertices $Z h^{n}, n \geq 2$. Furthermore, $\mathcal{S}_{2 D}(h)$ induces, at 1-loop, corrections to the Higgs gauge-boson couplings that are bounded by the strong experimental limits on fermionic EDMs, as discussed in Ref. [25]. For this reason, it is considered as a NLO operator, similarly to $\mathcal{P}_{T}(h)$.

Finally, the two operators $\mathcal{P}_{\widetilde{W} W W}(h)$ and $\mathcal{P}_{\widetilde{G} G G}(h)$ are the CP odd counterparts of $\mathcal{P}_{W W W}(h)$ and $\mathcal{P}_{G G G}(h)$; comments similar to those given for the latter apply here too.

\subsection{NLO basis: fermionic sector $\Delta \mathscr{L}_{\text {fer }}$}

The fermionic Lagrangian at NLO is constituted by singlecurrent operators with up to two derivatives and by fourfermion operators. Flavour indices are left implicit, unless necessary for the discussion. This section presents a set of independent terms that completes the NLO basis in the bosonic sector $\Delta \mathscr{L}_{\text {bos }}$ : some redundant structures have been removed using the EOMs, as detailed in Appendix D. Only baryon and lepton number conserving operators are considered (see Ref. [44] for the baryon and lepton number violating basis). Moreover, as already stated in the previous sections, right-handed neutrinos are not considered in the present description. Their inclusion in the spectrum would require an extension of the basis presented in this section, with the addition of the operators in Appendix A.
The numbering of the functions $\mathcal{F}_{i}(h)$ is dropped in the following for brevity. The Pauli matrices that act on the $S U(2)_{L}$ components are denoted by $\sigma^{i}$, while the Gell-Mann matrices that contract colour indices are indicated by $\lambda^{A}$. Whenever they are not specified, the colour (uppercase) and isospin (lowercase) contractions are understood to be diagonal. Flavour contractions are also assumed to be diagonal. The tensor structure $\sigma^{\mu \nu}$ entering the dipole operators is defined as $\sigma^{\mu \nu}=\frac{i}{2}\left[\gamma^{\mu}, \gamma^{\nu}\right]$. Finally, the mark CP on the left of an operator indicates that it is intrinsically CP odd.

\subsubsection{Single fermionic current $\Delta \mathscr{L}_{2 F}$}

The operators with a single fermionic current and up to two derivatives (including those in $\mathbf{V}_{\mu}$ ) are contained in the Lagrangian

$$
\begin{aligned}
\Delta \mathscr{L}_{2 F}= & \sum_{j=1}^{8} n_{j}^{\mathcal{Q}} \mathcal{N}_{j}^{\mathcal{Q}}+\sum_{j=9}^{28} \frac{1}{\Lambda}\left(n_{j}^{\mathcal{Q}}+i \tilde{n}_{j}^{\mathcal{Q}}\right) \mathcal{N}_{j}^{\mathcal{Q}} \\
& +\sum_{j=29}^{36} \frac{4 \pi}{\Lambda}\left(n_{j}^{\mathcal{Q}}+i \tilde{n}_{j}^{\mathcal{Q}}\right) \mathcal{N}_{j}^{\mathcal{Q}} \\
& +\sum_{j=1}^{2} n_{j}^{\ell} \mathcal{N}_{j}^{\ell}+\sum_{j=3}^{11} \frac{1}{\Lambda}\left(n_{j}^{\ell}+i \tilde{n}_{j}^{\ell}\right) \mathcal{N}_{j}^{\ell} \\
& +\sum_{j=12}^{14} \frac{4 \pi}{\Lambda}\left(n_{j}^{\ell}+i \tilde{n}_{j}^{\ell}\right) \mathcal{N}_{j}^{\ell}+\text { h.c. },
\end{aligned}
$$

where we recall that the coefficients $n_{j}^{\mathcal{Q}}, n_{j}^{\ell}, \tilde{n}_{j}^{\mathcal{Q}}, \tilde{n}_{j}^{\ell}$ are real and smaller than unity.

The terms with two derivatives have overall canonical mass dimension 5 and are therefore suppressed by $\Lambda^{-1}$. Moreover, they necessarily require chirality-flipping (scalar or tensor) Lorentz structures. These structures do not have definite CP character, as the scalar $(\bar{\psi} \psi)$ and pseudo-scalar $\left(\bar{\psi} i \gamma_{5} \psi\right)$ contractions have opposite parity. As a consequence, each $S U(2)$ structure yields two contributions with opposite CP properties, which have been parameterised by two independent real coefficients: for the quark bilinears, the terms $n_{j}^{\mathcal{Q}}\left(\mathcal{N}_{j}^{\mathcal{Q}}+\right.$ h.c. $)$ with the $\mathcal{N}_{j}^{\mathcal{Q}}$, s defined below are $\mathrm{CP}$ even, while the combinations $\tilde{n}_{j}^{\mathcal{Q}}\left(i \mathcal{N}_{j}^{\mathcal{Q}}+\right.$ h.c. $)$ are CP odd. A similar notation has been adopted for the lepton bilinears.

\section{Quark current operators}

All the non-redundant terms that can be constructed coupling one derivative or one chiral vector field $\mathbf{V}_{\mu}$ to a fermionic bilinear necessarily have a vector-axial Lorentz structure, that preserves chirality. For the quarks case, they are:

$\mathcal{N}_{1}^{\mathcal{Q}}(h) \equiv i \bar{Q}_{L} \gamma_{\mu} \mathbf{V}^{\mu} Q_{L} \mathcal{F}$, 


$$
\begin{gathered}
\mathcal{N}_{2}^{\mathcal{Q}}(h) \equiv i \bar{Q}_{R} \gamma_{\mu} \mathbf{U}^{\dagger} \mathbf{V}^{\mu} \mathbf{U} Q_{R} \mathcal{F}, \\
\text { CP } \mathcal{N}_{3}^{\mathcal{Q}}(h) \equiv \bar{Q}_{L} \gamma_{\mu}\left[\mathbf{V}^{\mu}, \mathbf{T}\right] Q_{L} \mathcal{F}, \\
\text { CP } \mathcal{N}_{4}^{\mathcal{Q}}(h) \equiv \bar{Q}_{R} \gamma_{\mu} \mathbf{U}^{\dagger}\left[\mathbf{V}^{\mu}, \mathbf{T}\right] \mathbf{U} Q_{R} \mathcal{F}, \\
\mathcal{N}_{5}^{\mathcal{Q}}(h) \equiv i \bar{Q}_{L} \gamma_{\mu}\left\{\mathbf{V}^{\mu}, \mathbf{T}\right\} Q_{L} \mathcal{F}, \\
\mathcal{N}_{6}^{\mathcal{Q}}(h) \equiv i \bar{Q}_{R} \gamma_{\mu} \mathbf{U}^{\dagger}\left\{\mathbf{V}^{\mu}, \mathbf{T}\right\} \mathbf{U} Q_{R} \mathcal{F}, \\
\mathcal{N}_{7}^{\mathcal{Q}}(h) \equiv i \bar{Q}_{L} \gamma_{\mu} \mathbf{T V}^{\mu} \mathbf{T} Q_{L} \mathcal{F}, \\
\mathcal{N}_{8}^{\mathcal{Q}}(h) \equiv i \bar{Q}_{R} \gamma_{\mu} \mathbf{U}^{\dagger} \mathbf{T} \mathbf{V}^{\mu} \mathbf{T} \mathbf{U} Q_{R} \mathcal{F} .
\end{gathered}
$$

Invariants with a derivative acting on a fermion field or on a $\mathcal{F}(h)$ function are redundant upon application of the EOMs and integration by parts, and they have therefore been removed from the final basis.

Operators with two derivatives require a fermionic current with an even number (zero or two) of gamma matrices: therefore only chirality-flipping Lorentz structures are allowed. All the operators with a scalar structure are required as counter-terms in the 1-loop renormalisation of $\mathscr{L}_{0}$ :

$\mathcal{N}_{9}^{\mathcal{Q}}(h) \equiv \bar{Q}_{L} \mathbf{U} Q_{R} \partial_{\mu} \mathcal{F} \partial^{\mu} \mathcal{F}^{\prime}$,

$\mathcal{N}_{10}^{\mathcal{Q}}(h) \equiv \bar{Q}_{L} \mathbf{T U} Q_{R} \partial_{\mu} \mathcal{F} \partial^{\mu} \mathcal{F}^{\prime}$,

$\mathcal{N}_{11}^{\mathcal{Q}}(h) \equiv \bar{Q}_{L} \mathbf{V}_{\mu} \mathbf{U} Q_{R} \partial^{\mu} \mathcal{F}$,

$\mathcal{N}_{12}^{\mathcal{Q}}(h) \equiv \bar{Q}_{L}\left\{\mathbf{V}_{\mu}, \mathbf{T}\right\} \mathbf{U} Q_{R} \partial^{\mu} \mathcal{F}$,

$\mathcal{N}_{13}^{\mathcal{Q}}(h) \equiv \bar{Q}_{L}\left[\mathbf{V}_{\mu}, \mathbf{T}\right] \mathbf{U} Q_{R} \partial^{\mu} \mathcal{F}$,

$\mathcal{N}_{14}^{\mathcal{Q}}(h) \equiv \bar{Q}_{L} \mathbf{T} \mathbf{V}_{\mu} \mathbf{T U} Q_{R} \partial^{\mu} \mathcal{F}$,

$\mathcal{N}_{15}^{\mathcal{Q}}(h) \equiv \bar{Q}_{L} \mathbf{V}_{\mu} \mathbf{V}^{\mu} \mathbf{U} Q_{R} \mathcal{F}$,

$\mathcal{N}_{16}^{\mathcal{Q}}(h) \equiv \bar{Q}_{L} \mathbf{V}_{\mu} \mathbf{V}^{\mu} \mathbf{T} \mathbf{U} Q_{R} \mathcal{F}$,

$\mathcal{N}_{17}^{\mathcal{Q}}(h) \equiv \bar{Q}_{L} \mathbf{T V}_{\mu} \mathbf{T V}^{\mu} \mathbf{U} Q_{R} \mathcal{F}$,

$\mathcal{N}_{18}^{\mathcal{Q}}(h) \equiv \bar{Q}_{L} \mathbf{T V}_{\mu} \mathbf{T V}^{\mu} \mathbf{T U} Q_{R} \mathcal{F}$,

$\mathcal{N}_{19}^{\mathcal{Q}}(h) \equiv \bar{Q}_{L} \mathbf{V}_{\mu} \mathbf{T V}^{\mu} \mathbf{U} Q_{R} \mathcal{F}$,

$\mathcal{N}_{20}^{\mathcal{Q}}(h) \equiv \bar{Q}_{L} \mathbf{V}_{\mu} \mathbf{T} \mathbf{V}^{\mu} \mathbf{T U} Q_{R} \mathcal{F}$.

Operators with tensor structure are also included in the NLO basis, although they are not needed to reabsorb the 1loop divergences of $\mathscr{L}_{0}$, as the loop diagrams that generate them in the EFT are finite. Nonetheless, these interactions may result from the (tree-level) exchange of a heavy BSM resonance and therefore they may be as relevant as those in the previous lists:

$\mathcal{N}_{21}^{\mathcal{Q}}(h) \equiv \bar{Q}_{L} \sigma^{\mu \nu} \mathbf{V}_{\mu} \mathbf{U} Q_{R} \partial_{\nu} \mathcal{F}$,

$\mathcal{N}_{22}^{\mathcal{Q}}(h) \equiv \bar{Q}_{L} \sigma^{\mu \nu}\left[\mathbf{V}_{\mu}, \mathbf{T}\right] \mathbf{U} Q_{R} \partial_{\nu} \mathcal{F}$,

$\mathcal{N}_{23}^{\mathcal{Q}}(h) \equiv \bar{Q}_{L} \sigma^{\mu v}\left\{\mathbf{V}_{\mu}, \mathbf{T}\right\} \mathbf{U} Q_{R} \partial_{\nu} \mathcal{F}$,

$\mathcal{N}_{24}^{\mathcal{Q}}(h) \equiv \bar{Q}_{L} \sigma^{\mu \nu} \mathbf{T} \mathbf{V}_{\mu} \mathbf{T U} Q_{R} \partial_{\nu} \mathcal{F}$,

$\mathcal{N}_{25}^{\mathcal{Q}}(h) \equiv \bar{Q}_{L} \sigma^{\mu \nu} \mathbf{V}_{\mu} \mathbf{T} \mathbf{V}_{\nu} \mathbf{U} Q_{R} \mathcal{F}$,

$\mathcal{N}_{26}^{\mathcal{Q}}(h) \equiv \bar{Q}_{L} \sigma^{\mu \nu} \mathbf{V}_{\mu} \mathbf{T} \mathbf{V}_{\nu} \mathbf{T U} Q_{R} \mathcal{F}$,

$\mathcal{N}_{27}^{\mathcal{Q}}(h) \equiv \bar{Q}_{L} \sigma^{\mu \nu}\left[\mathbf{V}_{\mu}, \mathbf{V}_{\nu}\right] \mathbf{U} Q_{R} \mathcal{F}$,
$\mathcal{N}_{28}^{\mathcal{Q}}(h) \equiv \bar{Q}_{L} \sigma^{\mu \nu}\left[\mathbf{V}_{\mu}, \mathbf{V}_{\nu}\right] \mathbf{T U} Q_{R} \mathcal{F}$,

$\mathcal{N}_{29}^{\mathcal{Q}}(h) \equiv i g^{\prime} \bar{Q}_{L} \sigma^{\mu v} \mathbf{U} Q_{R} B_{\mu \nu} \mathcal{F}$,

$\mathcal{N}_{30}^{\mathcal{Q}}(h) \equiv i g^{\prime} \bar{Q}_{L} \sigma^{\mu \nu} \mathbf{T U} Q_{R} B_{\mu \nu} \mathcal{F}$,

$\mathcal{N}_{31}^{\mathcal{Q}}(h) \equiv i g_{s} \bar{Q}_{L} \sigma^{\mu \nu} \mathcal{G}_{\mu \nu} \mathbf{U} Q_{R} \mathcal{F}$,

$\mathcal{N}_{32}^{\mathcal{Q}}(h) \equiv i g_{s} \bar{Q}_{L} \sigma^{\mu \nu} \mathcal{G}_{\mu \nu} \mathbf{T U} Q_{R} \mathcal{F}$,

$\mathcal{N}_{33}^{\mathcal{Q}}(h) \equiv i g \bar{Q}_{L} \sigma^{\mu \nu} W_{\mu \nu} \mathbf{U} Q_{R} \mathcal{F}$,

$\mathcal{N}_{34}^{\mathcal{Q}}(h) \equiv i g \bar{Q}_{L} \sigma^{\mu \nu}\left\{W_{\mu \nu}, \mathbf{T}\right\} \mathbf{U} Q_{R} \mathcal{F}$,

$\mathcal{N}_{35}^{\mathcal{Q}}(h) \equiv i g \bar{Q}_{L} \sigma^{\mu \nu}\left[W_{\mu \nu}, \mathbf{T}\right] \mathbf{U} Q_{R} \mathcal{F}$,

$\mathcal{N}_{36}^{\mathcal{Q}}(h) \equiv i g \bar{Q}_{L} \sigma^{\mu \nu} \mathbf{T} W_{\mu \nu} \mathbf{T U} Q_{R} \mathcal{F}$.

\section{Leptonic current operators}

Leptonic bilinears can be constructed along the same lines as the quark ones. The absence of right-handed neutrinos, however, reduces notably the number of independent invariants. Making use of Eq. (D.14), only two independent operators can be constructed with the insertion of a single derivative or $\mathbf{V}_{\mu}$ :

$$
\begin{aligned}
\mathscr{\mathcal { N } _ { 1 } ^ { \ell }}(h) & \equiv \bar{L}_{L} \gamma_{\mu}\left[\mathbf{V}^{\mu}, \mathbf{T}\right] L_{L} \mathcal{F}, \\
\mathcal{N}_{2}^{\ell}(h) & \equiv i \bar{L}_{R} \gamma_{\mu} \mathbf{U}^{\dagger}\left\{\mathbf{V}^{\mu}, \mathbf{T}\right\} \mathbf{U} L_{R} \mathcal{F} .
\end{aligned}
$$

Notice that, if flavour effects are also taken into consideration, two other structures should be considered:

$i \bar{L}_{L i} \gamma_{\mu} \mathbf{V}^{\mu} L_{L j} \mathcal{F}, \quad i \bar{L}_{L i} \gamma_{\mu}\left\{\mathbf{T}, \mathbf{V}^{\mu}\right\} L_{L j} \mathcal{F}$.

only for the case with $i \neq j$. Indeed, as shown in Eq. (D.14), the flavour-diagonal contractions do not represent independent terms as they are related via EOMs to bosonic operators that have been retained in the basis.

With two derivatives, two $\mathbf{V}_{\mu}$ or a combination of them, the following structures can be constructed:

$\mathcal{N}_{3}^{\ell}(h) \equiv \bar{L}_{L} \mathbf{U} L_{R} \partial_{\mu} \mathcal{F} \partial^{\mu} \mathcal{F}^{\prime}$,

$\mathcal{N}_{4}^{\ell}(h) \equiv \bar{L}_{L}\left\{\mathbf{V}_{\mu}, \mathbf{T}\right\} \mathbf{U} L_{R} \partial^{\mu} \mathcal{F}$,

$\mathcal{N}_{5}^{\ell}(h) \equiv \bar{L}_{L}\left[\mathbf{V}_{\mu}, \mathbf{T}\right] \mathbf{U} L_{R} \partial^{\mu} \mathcal{F}$,

$\mathcal{N}_{6}^{\ell}(h) \equiv \bar{L}_{L} \mathbf{V}_{\mu} \mathbf{V}^{\mu} \mathbf{U} L_{R} \mathcal{F}$,

$\mathcal{N}_{7}^{\ell}(h) \equiv \bar{L}_{L} \mathbf{T} \mathbf{V}_{\mu} \mathbf{T} \mathbf{V}^{\mu} \mathbf{U} L_{R} \mathcal{F}$,

$\mathcal{N}_{8}^{\ell}(h) \equiv \bar{L}_{L} \sigma^{\mu v}\left[\mathbf{V}_{\mu}, \mathbf{T}\right] \mathbf{U} L_{R} \partial_{\nu} \mathcal{F}$,

$\mathcal{N}_{9}^{\ell}(h) \equiv \bar{L}_{L} \sigma^{\mu \nu}\left\{\mathbf{V}_{\mu}, \mathbf{T}\right\} \mathbf{U} L_{R} \partial_{v} \mathcal{F}$,

$\mathcal{N}_{10}^{\ell}(h) \equiv \bar{L}_{L} \sigma^{\mu \nu} \mathbf{V}_{\mu} \mathbf{T} \mathbf{V}_{\nu} \mathbf{U} L_{R} \mathcal{F}$,

$\mathcal{N}_{11}^{\ell}(h) \equiv \bar{L}_{L} \sigma^{\mu \nu}\left[\mathbf{V}_{\mu}, \mathbf{V}_{\nu}\right] \mathbf{U} L_{R} \mathcal{F}$,

$\mathcal{N}_{12}^{\ell}(h) \equiv i g^{\prime} \bar{L}_{L} \sigma^{\mu \nu} \mathbf{U} L_{R} B_{\mu \nu} \mathcal{F}$,

$\mathcal{N}_{13}^{\ell}(h) \equiv i g \bar{L}_{L} \sigma^{\mu \nu} W_{\mu \nu} \mathbf{U} L_{R} \mathcal{F}$,

$\mathcal{N}_{14}^{\ell}(h) \equiv i g \bar{L}_{L} \sigma^{\mu \nu}\left[W_{\mu \nu}, \mathbf{T}\right] \mathbf{U} L_{R} \mathcal{F}$.

where, as explained above, all these operators are required as counter-terms in the 1-loop renormalisation of $\mathscr{L}_{0}$ with the exception of those with tensor structure, that correspond 
to finite contributions. It is also worth recalling that all the chirality-flipping structures listed here are $\mathrm{CP}$ even in the combination $\left(\mathcal{N}_{j}^{\ell}+\right.$ h.c. $)$ but independent $\mathrm{CP}$ violating terms of the form $\left(i \mathcal{N}_{j}^{\ell}+\right.$ h.c. $)$ should also be considered.

\subsubsection{Four-fermion operators $\Delta \mathscr{L}_{4 F}$}

Four-fermion operators can be classified into four-quark, four-lepton and two-quark-two-lepton sets. The overall Lagrangian reads

$$
\begin{aligned}
\Delta \mathscr{L}_{4 F}= & \frac{(4 \pi)^{2}}{\Lambda^{2}}\left[\sum_{j=1}^{8}\left(r_{j}^{\mathcal{Q}}+i \tilde{r}_{j}^{\mathcal{Q}}\right) R_{j}^{\mathcal{Q}}+\sum_{j=9}^{26} r_{j}^{\mathcal{O}} R_{j}^{\mathcal{Q}}\right. \\
& +\left(r_{1}^{\ell}+i \tilde{r}_{1}^{\ell}\right) R_{1}^{\ell}+\sum_{j=2}^{7} r_{j}^{\ell} R_{j}^{\ell} \\
& \left.+\sum_{j=1}^{6}\left(r_{j}^{\mathcal{Q} \ell}+i \tilde{r}_{j}^{\mathcal{Q} \ell}\right) R_{j}^{\mathcal{Q} \ell}+\sum_{j=7}^{23} r_{j}^{\mathcal{Q} \ell} R_{j}^{\mathcal{Q} \ell}+\text { h.c. }\right] .
\end{aligned}
$$

Details of the construction and reduction of this subset of operators can be found in Appendix C.3. As for the bilinears case, the chirality-flipping contractions $\left(\bar{\psi}_{L} \psi_{R}\right)\left(\bar{\psi}_{L} \psi_{R}\right)$ listed here are $\mathrm{CP}$ even in the combination $\left(R_{j}^{f}+\right.$ h.c.) but independent $\mathrm{CP}$ violating terms of the form $\left(i R_{j}^{f}+\right.$ h.c. $)$ should also be considered.

\section{Pure quark operators}

The only four-quark operators required to remove divergences originating at one loop are the following:

$$
\begin{aligned}
& R_{1}^{\mathcal{Q}}(h) \equiv\left(\bar{Q}_{L} \mathbf{U} Q_{R}\right)\left(\bar{Q}_{L} \mathbf{U} Q_{R}\right) \mathcal{F}, \\
& R_{2}^{\mathcal{Q}}(h) \equiv\left(\bar{Q}_{L} \sigma^{i} \mathbf{U} Q_{R}\right)\left(\bar{Q}_{L} \sigma^{i} \mathbf{U} Q_{R}\right) \mathcal{F}, \\
& R_{3}^{\mathcal{Q}}(h) \equiv\left(\bar{Q}_{L} \mathbf{U} Q_{R}\right)\left(\bar{Q}_{L} \mathbf{T} \mathbf{U} Q_{R}\right) \mathcal{F}, \\
& R_{4}^{\mathcal{Q}}(h) \equiv\left(\bar{Q}_{L} \mathbf{T} \mathbf{U} Q_{R}\right)\left(\bar{Q}_{L} \mathbf{T} \mathbf{U} Q_{R}\right) \mathcal{F}, \\
& R_{5}^{\mathcal{Q}}(h) \equiv\left(\bar{Q}_{L} \lambda^{A} \mathbf{U} Q_{R}\right)\left(\bar{Q}_{L} \lambda^{A} \mathbf{U} Q_{R}\right) \mathcal{F}, \\
& R_{6}^{\mathcal{Q}}(h) \equiv\left(\bar{Q}_{L} \lambda^{A} \sigma^{i} \mathbf{U} Q_{R}\right)\left(\bar{Q}_{L} \lambda^{A} \sigma^{i} \mathbf{U} Q_{R}\right) \mathcal{F} \\
& R_{7}^{\mathcal{Q}}(h) \equiv\left(\bar{Q}_{L} \lambda^{A} \mathbf{U} Q_{R}\right)\left(\bar{Q}_{L} \lambda^{A} \mathbf{T} \mathbf{U} Q_{R}\right) \mathcal{F}, \\
& R_{8}^{\mathcal{Q}}(h) \equiv\left(\bar{Q}_{L} \lambda^{A} \mathbf{T} \mathbf{U} Q_{R}\right)\left(\bar{Q}_{L} \lambda^{A} \mathbf{T} \mathbf{U} Q_{R}\right) \mathcal{F} .
\end{aligned}
$$

A large number of additional structures can be constructed, that are listed below and included in the basis. Although they do not correspond to counter-terms in the renormalisation of $\mathscr{L}_{0}$, they are potentially generated by the exchange of BSM resonances:

$$
\begin{aligned}
& R_{9}^{\mathcal{Q}}(h) \equiv\left(\bar{Q}_{L} \gamma_{\mu} Q_{L}\right)\left(\bar{Q}_{L} \gamma^{\mu} Q_{L}\right) \mathcal{F}, \\
& R_{10}^{\mathcal{Q}}(h) \equiv\left(\bar{Q}_{L} \gamma_{\mu} Q_{L}\right)\left(\bar{Q}_{L} \gamma^{\mu} \mathbf{T} Q_{L}\right) \mathcal{F}, \\
& R_{11}^{\mathcal{Q}}(h) \equiv\left(\bar{Q}_{L} \gamma_{\mu} \mathbf{T} Q_{L}\right)\left(\bar{Q}_{L} \gamma^{\mu} \mathbf{T} Q_{L}\right) \mathcal{F}, \\
& R_{12}^{\mathcal{Q}}(h) \equiv\left(\bar{Q}_{L} \gamma_{\mu} \sigma^{j} Q_{L}\right)\left(\bar{Q}_{L} \gamma^{\mu} \sigma^{j} Q_{L}\right) \mathcal{F}, \\
& R_{13}^{\mathcal{Q}}(h) \equiv\left(\bar{Q}_{R} \gamma_{\mu} Q_{R}\right)\left(\bar{Q}_{R} \gamma^{\mu} Q_{R}\right) \mathcal{F}, \\
& R_{14}^{\mathcal{Q}}(h) \equiv\left(\bar{Q}_{R} \gamma_{\mu} Q_{R}\right)\left(\bar{Q}_{R} \gamma^{\mu} \mathbf{U}^{\dagger} \mathbf{T} \mathbf{U} Q_{R}\right) \mathcal{F}, \\
& R_{15}^{\mathcal{Q}}(h) \equiv\left(\bar{Q}_{R} \gamma_{\mu} \mathbf{U}^{\dagger} \mathbf{T} \mathbf{U} Q_{R}\right)\left(\bar{Q}_{R} \gamma^{\mu} \mathbf{U}^{\dagger} \mathbf{T} \mathbf{U} Q_{R}\right) \mathcal{F}, \\
& R_{16}^{\mathcal{Q}}(h) \equiv\left(\bar{Q}_{R} \gamma_{\mu} \sigma^{j} Q_{R}\right)\left(\bar{Q}_{R} \gamma^{\mu} \mathbf{U}^{\dagger} \sigma^{j} \mathbf{U} Q_{R}\right) \mathcal{F}, \\
& R_{17}^{\mathcal{Q}}(h) \equiv\left(\bar{Q}_{L} \gamma_{\mu} Q_{L}\right)\left(\bar{Q}_{R} \gamma^{\mu} Q_{R}\right) \mathcal{F} \\
& R_{18}^{\mathcal{Q}}(h) \equiv\left(\bar{Q}_{L} \gamma_{\mu} Q_{L}\right)\left(\bar{Q}_{R} \gamma^{\mu} \mathbf{U}^{\dagger} \mathbf{T} \mathbf{U} Q_{R}\right) \mathcal{F}, \\
& R_{19}^{\mathcal{Q}}(h) \equiv\left(\bar{Q}_{L} \gamma^{\mu} \mathbf{T} Q_{L}\right)\left(\bar{Q}_{R} \gamma_{\mu} Q_{R}\right) \mathcal{F}, \\
& R_{20}^{\mathcal{Q}}(h) \equiv\left(\bar{Q}_{L} \gamma_{\mu} \mathbf{T} Q_{L}\right)\left(\bar{Q}_{R} \gamma^{\mu} \mathbf{U}^{\dagger} \mathbf{T} \mathbf{U} Q_{R}\right) \mathcal{F}, \\
& R_{21}^{\mathcal{Q}}(h) \equiv\left(\bar{Q}_{L} \gamma_{\mu} \sigma^{i} Q_{L}\right)\left(\bar{Q}_{R} \gamma^{\mu} \mathbf{U}^{\dagger} \sigma^{i} \mathbf{U} Q_{R}\right) \mathcal{F}, \\
& R_{22}^{\mathcal{Q}}(h) \equiv\left(\bar{Q}_{L} \gamma_{\mu} \lambda^{A} Q_{L}\right)\left(\bar{Q}_{R} \gamma^{\mu} \lambda^{A} Q_{R}\right) \mathcal{F}, \\
& R_{23}^{\mathcal{Q}}(h) \equiv\left(\bar{Q}_{L} \gamma_{\mu} \lambda^{A} Q_{L}\right)\left(\bar{Q}_{R} \gamma^{\mu} \lambda^{A} \mathbf{U}^{\dagger} \mathbf{T} \mathbf{U} Q_{R}\right) \mathcal{F}, \\
& R_{24}^{\mathcal{Q}}(h) \equiv\left(\bar{Q}_{L} \gamma^{\mu} \lambda^{A} \mathbf{T} Q_{L}\right)\left(\bar{Q}_{R} \gamma_{\mu} \lambda^{A} Q_{R}\right) \mathcal{F}, \\
& R_{25}^{\mathcal{Q}}(h) \equiv\left(\bar{Q}_{L} \gamma_{\mu} \lambda^{A} \mathbf{T} Q_{L}\right)\left(\bar{Q}_{R} \gamma^{\mu} \lambda^{A} \mathbf{U}^{\dagger} \mathbf{T} \mathbf{U} Q_{R}\right) \mathcal{F}, \\
& R_{26}^{\mathcal{Q}}(h) \equiv\left(\bar{Q}_{L} \gamma_{\mu} \lambda^{A} \sigma^{i} Q_{L}\right)\left(\bar{Q}_{R} \gamma^{\mu} \lambda^{A} \mathbf{U}^{\dagger} \sigma^{i} \mathbf{U} Q_{R}\right) \mathcal{F} .
\end{aligned}
$$

\section{Pure leptonic operators}

The set of independent four-lepton operators is considerably smaller than that with four quarks, due to the absence of righthanded neutrinos and of colour charges. Only one operator is required as a 1-loop counter-term:

$$
R_{1}^{\ell}(h) \equiv\left(\bar{L}_{L} \mathbf{U} L_{R}\right)\left(\bar{L}_{L} \mathbf{U} L_{R}\right) \mathcal{F} .
$$

Six additional structures, that are not required as counterterms, complete the list of possible invariants:

$$
\begin{aligned}
& R_{2}^{\ell}(h) \equiv\left(\bar{L}_{L} \gamma_{\mu} L_{L}\right)\left(\bar{L}_{L} \gamma^{\mu} L_{L}\right) \mathcal{F}, \\
& R_{3}^{\ell}(h) \equiv\left(\bar{L}_{R} \gamma_{\mu} L_{R}\right)\left(\bar{L}_{R} \gamma^{\mu} L_{R}\right) \mathcal{F}, \\
& R_{4}^{\ell}(h) \equiv\left(\bar{L}_{L} \gamma_{\mu} L_{L}\right)\left(\bar{L}_{L} \gamma^{\mu} \mathbf{T} L_{L}\right) \mathcal{F}, \\
& R_{5}^{\ell}(h) \equiv\left(\bar{L}_{L} \gamma_{\mu} \mathbf{T} L_{L}\right)\left(\bar{L}_{L} \gamma^{\mu} \mathbf{T} L_{L}\right) \mathcal{F}, \\
& R_{6}^{\ell}(h) \equiv\left(\bar{L}_{L} \gamma_{\mu} L_{L}\right)\left(\bar{L}_{R} \gamma^{\mu} L_{R}\right) \mathcal{F}, \\
& R_{7}^{\ell}(h) \equiv\left(\bar{L}_{L} \gamma^{\mu} \mathbf{T} L_{L}\right)\left(\bar{L}_{R} \gamma_{\mu} L_{R}\right) \mathcal{F} .
\end{aligned}
$$

\section{Mixed quark-lepton operators}

Finally, barring any $B$ or $L$ violation effects, mixed fourfermion operators can only contain two quarks and two leptons in either of the current structures $\bar{L} L \bar{Q} Q$ and $\bar{L} Q \bar{Q} L$.

Among the constructed invariants, the following are required to reabsorb 1-loop divergences: 
$R_{1}^{\mathcal{Q} \ell}(h) \equiv\left(\bar{L}_{L} \mathbf{U} L_{R}\right)\left(\bar{Q}_{L} \mathbf{U} Q_{R}\right) \mathcal{F}$,

$R_{2}^{\mathcal{Q}}(h) \equiv\left(\bar{L}_{L} \mathbf{U} Q_{R}\right)\left(\bar{Q}_{L} \mathbf{U} L_{R}\right) \mathcal{F}$,

$R_{3}^{\mathcal{Q} \ell}(h) \equiv\left(\bar{L}_{L} \mathbf{U} L_{R}\right)\left(\bar{Q}_{L} \mathbf{T U} Q_{R}\right) \mathcal{F}$,

$R_{4}^{\mathcal{Q} \ell}(h) \equiv\left(\bar{L}_{L} \mathbf{T U} Q_{R}\right)\left(\bar{Q}_{L} \mathbf{U} L_{R}\right) \mathcal{F}$,

$R_{5}^{\mathcal{Q} \ell}(h) \equiv\left(\bar{L}_{L} \sigma^{i} \mathbf{U} L_{R}\right)\left(\bar{Q}_{L} \sigma^{i} \mathbf{U} Q_{R}\right) \mathcal{F}$,

$R_{6}^{\mathcal{Q} \ell}(h) \equiv\left(\bar{L}_{L} \sigma^{i} \mathbf{U} Q_{R}\right)\left(\bar{Q}_{L} \sigma^{i} \mathbf{U} L_{R}\right) \mathcal{F}$,

while the remaining correspond to finite diagrams and are included for completeness:

$R_{7}^{\mathcal{Q} \ell}(h) \equiv\left(\bar{L}_{L} \gamma_{\mu} L_{L}\right)\left(\bar{Q}_{L} \gamma^{\mu} Q_{L}\right) \mathcal{F}$,

$R_{8}^{\mathcal{Q} \ell}(h) \equiv\left(\bar{L}_{R} \gamma_{\mu} L_{R}\right)\left(\bar{Q}_{R} \gamma^{\mu} Q_{R}\right) \mathcal{F}$,

$R_{9}^{\mathcal{Q} \ell}(h) \equiv\left(\bar{L}_{L} \gamma_{\mu} L_{L}\right)\left(\bar{Q}_{L} \gamma^{\mu} \mathbf{T} Q_{L}\right) \mathcal{F}$,

$R_{10}^{\mathcal{Q} \ell}(h) \equiv\left(\bar{L}_{R} \gamma_{\mu} L_{R}\right)\left(\bar{Q}_{R} \gamma^{\mu} \mathbf{U}^{\dagger} \mathbf{T} \mathbf{U} Q_{R}\right) \mathcal{F}$,

$R_{11}^{\mathcal{Q} \ell}(h) \equiv\left(\bar{L}_{L} \gamma_{\mu} \mathbf{T} L_{L}\right)\left(\bar{Q}_{L} \gamma^{\mu} Q_{L}\right) \mathcal{F}$,

$R_{12}^{\mathcal{Q} \ell}(h) \equiv\left(\bar{L}_{L} \gamma_{\mu} \mathbf{T} L_{L}\right)\left(\bar{Q}_{L} \gamma^{\mu} \mathbf{T} Q_{L}\right) \mathcal{F}$,

$R_{13}^{\mathcal{Q} \ell}(h) \equiv\left(\bar{L}_{L} \gamma_{\mu} \sigma^{i} L_{L}\right)\left(\bar{Q}_{L} \gamma^{\mu} \sigma^{i} Q_{L}\right) \mathcal{F}$,

$R_{14}^{\mathcal{Q} \ell}(h) \equiv\left(\bar{L}_{L} \gamma_{\mu} L_{L}\right)\left(\bar{Q}_{R} \gamma^{\mu} Q_{R}\right) \mathcal{F}$,

$R_{15}^{\mathcal{Q} \ell}(h) \equiv\left(\bar{Q}_{L} \gamma_{\mu} Q_{L}\right)\left(\bar{L}_{R} \gamma^{\mu} L_{R}\right) \mathcal{F}$,

$R_{16}^{\mathcal{Q} \ell}(h) \equiv\left(\bar{L}_{L} \gamma^{\mu} \mathbf{T} L_{L}\right)\left(\bar{Q}_{R} \gamma_{\mu} Q_{R}\right) \mathcal{F}$,

$R_{17}^{\mathcal{Q} \ell}(h) \equiv\left(\bar{Q}_{L} \gamma_{\mu} \mathbf{T} Q_{L}\right)\left(\bar{L}_{R} \gamma^{\mu} L_{R}\right) \mathcal{F}$,

$R_{18}^{\mathcal{Q} \ell}(h) \equiv\left(\bar{L}_{L} \gamma_{\mu} L_{L}\right)\left(\bar{Q}_{R} \gamma^{\mu} \mathbf{U}^{\dagger} \mathbf{T} \mathbf{U} Q_{R}\right) \mathcal{F}$,

$R_{19}^{\mathcal{Q} \ell}(h) \equiv\left(\bar{L}_{L} \gamma^{\mu} \mathbf{T} L_{L}\right)\left(\bar{Q}_{R} \gamma_{\mu} \mathbf{U}^{\dagger} \mathbf{T} \mathbf{U} Q_{R}\right) \mathcal{F}$,

$R_{20}^{\mathcal{Q} \ell}(h) \equiv\left(\bar{L}_{L} \gamma^{\mu} \sigma^{j} L_{L}\right)\left(\bar{Q}_{R} \gamma_{\mu} \mathbf{U}^{\dagger} \sigma^{j} \mathbf{U} Q_{R}\right) \mathcal{F}$,

$R_{21}^{\mathcal{Q} \ell}(h) \equiv\left(\bar{Q}_{L} \gamma_{\mu} L_{L}\right)\left(\bar{L}_{R} \gamma^{\mu} Q_{R}\right) \mathcal{F}$,

$R_{22}^{\mathcal{Q} \ell}(h) \equiv\left(\bar{Q}_{L} \gamma_{\mu} \mathbf{T} L_{L}\right)\left(\bar{L}_{R} \gamma^{\mu} Q_{R}\right) \mathcal{F}$,

$R_{23}^{\mathcal{Q} \ell}(h) \equiv\left(\bar{Q}_{L} \gamma^{\mu} \sigma^{j} L_{L}\right)\left(\bar{L}_{R} \gamma_{\mu} \mathbf{U}^{\dagger} \sigma^{j} \mathbf{U} Q_{R}\right) \mathcal{F}$.

\subsection{Comparison with the SMEFT basis}

The comparison with the SMEFT is crucial for the identification of signals able to shed some light on the Higgs nature.

For the bosonic sector, the relation between the HEFT and its linear counterpart has already been identified in Ref. [24], adopting the so-called HISZ basis [45,46], which is also used in Refs. [47-49]. Those results still hold here, up to the fact that some operators have been traded for fermionic ones: the correspondence is summarised in Table 1, where the relation to the basis of Ref. [7] is also reported. The fermionic sector of the HEFT has also been matched with the linear bases of Refs. [7,47-49], as indicated in Table 2.

It is worth pointing out a few points that should be kept into account when performing this comparison:

- In the HEFT, right-handed fermions are grouped in the $S U(2)_{R}$ doublets, $L_{R}$ and $Q_{R}$, and the different com- ponents of each bilinear fermionic structure are disentangled inserting $\mathbf{U}^{\dagger} \mathbf{T} \mathbf{U}=\sigma^{3}$ or $\mathbf{U}^{\dagger} \sigma^{j} \mathbf{U}$. Each linear operator, written in the traditional notation, is then easily matched with a linear combination of HEFT invariants.

- The adimensional scalar field $\mathbf{T}$ corresponds, in the linear context, to a quadratic combination of Higgs doublets. As a consequence, the counterparts of fermionic invariants containing $\mathbf{T}$ are mostly linear operators of dimension $d>6$, which are therefore not present in the list of Refs. $[7,48,49]$.

The insertions of $\mathbf{T}$ into right-handed currents, mentioned in the previous point, represent an exception. In fact, in these cases $\mathbf{T}$ appears in the combination $\mathbf{U}^{\dagger} \mathbf{T U}=\sigma^{3}$, that does not contain any field and in fact is not associated to dimensional objects in the linear language.

- The adimensionality of $\mathbf{T}$ also leads to the presence of $\mathrm{CP}$-odd operators in $\Delta \mathscr{L}$, whose corresponding structures in the SMEFT would appear only at $d>6$. An example is the operator $\mathcal{N}_{3}^{\mathcal{Q}}(h)$ that has been already studied in Ref. [23,37] for its impact on flavour physics.

- The two-derivative object $\mathbf{V}_{\mu} \mathbf{V}^{\mu}$ is typically described, in the SMEFT, by a quantity proportional to $D_{\mu} \Phi^{\dagger} D^{\mu} \Phi$, which has canonical dimension 4 . Thus, fermionic bilinears containing this structure correspond to SMEFT operators with $d \geq 7$.

Tables 1 and 2 summarise the relations between operators of the HEFT, defined in the previous section, and those of the SMEFT from Refs. [7,48,49]. The only differ-

Table 1 Correspondence between the SMEFT operators from Refs. $[7,48,49]$, and the HEFT terms presented here for the bosonic sector. The - refers to the absence of an equivalent operator. The use of the " $\mathcal{Q} i$ " notation for the second column means that a particular operator does not explicitly appear in Refs. $[48,49]$, but it anyway enters the SMEFT basis and is defined as in Ref. [7]. Numerical coefficients and signs in the combinations of the HEFT operators are not indicated

\begin{tabular}{|c|c|c|c|c|c|}
\hline Ref. [7] & $\begin{array}{l}\text { Refs. } \\
{[48,49]}\end{array}$ & HEFT & Ref. [7] & $\begin{array}{l}\text { Refs. } \\
{[48,49]}\end{array}$ & HEFT \\
\hline $\mathcal{Q}_{\varphi}$ & $\mathcal{O}_{\Phi, 3}$ & scalar pot. & $\mathcal{Q}_{\varphi} \square$ & $\mathcal{O}_{\Phi_{2}}$ & $\mathcal{F}_{C}+\mathcal{F}_{Y}\left(\mathcal{P}_{H}\right)$ \\
\hline $\mathcal{Q}_{\varphi D}$ & $\mathcal{O}_{\Phi, 1}$ & $\mathcal{P}_{T}$ & $\mathcal{Q}_{\varphi G}$ & $\mathcal{O}_{G G}$ & $\mathcal{P}_{G}$ \\
\hline $\mathcal{Q}_{\varphi W}$ & $\mathcal{O}_{W W}$ & $\mathcal{P}_{W}$ & $\mathcal{Q}_{\varphi B}$ & $\mathcal{O}_{B B}$ & $\mathcal{P}_{B}$ \\
\hline $\mathcal{Q}_{\varphi W B}$ & $\mathcal{O}_{B W}$ & $\mathcal{P}_{1}$ & - & $\mathcal{O}_{B}$ & $\mathcal{P}_{2}+\mathcal{P}_{4}$ \\
\hline- & $\mathcal{O}_{W}$ & $\mathcal{P}_{3}+\mathcal{P}_{5}$ & & & \\
\hline $\mathcal{Q}_{G}$ & $" \mathcal{Q}_{G} "$ & $\mathcal{P}_{G G G}$ & $\mathcal{Q}_{W}$ & $\mathcal{O}_{W W W}$ & $\mathcal{P}_{W W W}$ \\
\hline $\mathcal{Q}_{\varphi} \widetilde{G}$ & $" \mathcal{Q}_{\varphi} \widetilde{G} "$ & $\mathcal{S}_{\tilde{G}}$ & $\mathcal{Q}_{\varphi \widetilde{B}}$ & $" \mathcal{Q}_{\varphi \widetilde{B}} "$ & $\mathcal{S}_{\tilde{B}}$ \\
\hline $\mathcal{Q}_{\varphi} \widetilde{W}$ & " $\mathcal{Q}_{\varphi} \widetilde{W} "$ & $\mathcal{S}_{\tilde{W}}$ & $\mathcal{Q}_{\varphi \widetilde{W} B}$ & $" \mathcal{Q}_{\varphi \widetilde{W} B} "$ & $\mathcal{S}_{1}$ \\
\hline $\mathcal{Q}_{\widetilde{G}}$ & $" \mathcal{Q}_{\widetilde{G}} "$ & $\mathcal{P}_{\widetilde{G} G G}$ & $\mathcal{Q}_{\widetilde{W}}$ & $" \mathcal{Q}_{\widetilde{W}} "$ & $\mathcal{P}_{\widetilde{W} W W}$ \\
\hline
\end{tabular}


Table 2 Correspondence between the SMEFT operators from Refs. $[7,48,49]$, and the HEFT terms presented here for the fermionic sector. The - refers to the absence of an equivalent operator. The use of the " $\mathcal{Q}_{i}$ " notation for the second column means that a particular operator does not explicitly appear in Refs. [48,49], but it anyway enters the SMEFT basis and is defined as in Ref. [7]. Flavour indices are omitted, unless explicitly indicated. Numerical coefficients and signs in the combinations of the HEFT operators are not indicated

\begin{tabular}{|c|c|c|c|c|c|}
\hline Ref. [7] & Refs. $[48,49]$ & HEFT & Ref. [7] & Refs. [48,49] & HEFT \\
\hline $\mathcal{Q}_{\varphi u}$ & $\mathcal{O}_{u \Phi}$ & $\mathcal{Y}_{U}(h)$ & $\mathcal{Q}_{\varphi e}$ & $\mathcal{O}_{e \Phi}$ & $\mathcal{Y}_{E}(h)$ \\
\hline $\mathcal{Q}_{\varphi d}$ & $\mathcal{O}_{d \Phi}$ & $\mathcal{Y}_{D}(h)$ & $\mathcal{Q}_{\varphi l, i i}^{(1)}$ & - & - \\
\hline $\mathcal{Q}_{\varphi q}^{(1)}$ & $\mathcal{O}_{\Phi Q}^{(1)}$ & $\mathcal{N}_{5}^{\mathcal{Q}}$ & $\mathcal{Q}_{\varphi l, i j}^{(1)}$ & $\mathcal{O}_{\Phi L, i j}^{(1)}$ & $i \bar{L}_{L_{i}} \gamma_{\mu}\left\{\mathbf{T}, \mathbf{V}^{\mu}\right\} L_{L_{j}} \mathcal{F}$ \\
\hline $\mathcal{Q}_{\varphi q}^{(3)}$ & $\mathcal{O}_{\Phi Q}^{(3)}$ & $\mathcal{N}_{1}^{\mathcal{Q}}$ & $\mathcal{Q}_{\varphi l, i i}^{(3)}$ & - & - \\
\hline $\mathcal{Q}_{\varphi u}$ & $\mathcal{O}_{\Phi u}^{(1)}$ & $\mathcal{N}_{2}^{\mathcal{Q}}+\mathcal{N}_{6}^{\mathcal{Q}}+\mathcal{N}_{8}^{\mathcal{Q}}$ & $\mathcal{Q}_{\varphi l, i j}^{(3)}$ & $\mathcal{O}_{\Phi L, i j}^{(3)}$ & $i \bar{L}_{L_{i}} \gamma_{\mu} \mathbf{V}^{\mu} L_{L_{j}} \mathcal{F}$ \\
\hline $\mathcal{Q}_{\varphi d}$ & $\mathcal{O}_{\Phi d}^{(1)}$ & $\mathcal{N}_{2}^{\mathcal{Q}}+\mathcal{N}_{6}^{\mathcal{Q}}+\mathcal{N}_{8}^{\mathcal{Q}}$ & $\mathcal{Q}_{\varphi e}$ & $\mathcal{O}_{\Phi e}^{(1)}$ & $\mathcal{N}_{2}^{\ell}$ \\
\hline $\mathcal{Q}_{\varphi \text { ud }}$ & $\mathcal{O}_{\text {Фud }}^{(1)}$ & $\mathcal{N}_{2}^{\mathcal{Q}}+\mathcal{N}_{8}^{\mathcal{Q}}$ & & & \\
\hline $\mathcal{Q}_{u G}$ & $" \mathcal{Q}_{u G} "$ & $\mathcal{N}_{31}^{\mathcal{Q}}+\mathcal{N}_{32}^{\mathcal{Q}}$ & & & \\
\hline $\mathcal{Q}_{d G}$ & $" \mathcal{Q}_{d G} "$ & $\mathcal{N}_{31}^{\mathcal{Q}}+\mathcal{N}_{32}^{\mathcal{Q}}$ & & & \\
\hline $\mathcal{Q}_{u W}$ & $" \mathcal{Q}_{u W} "$ & $\mathcal{N}_{33}^{\mathcal{Q}}+\mathcal{N}_{34}^{\mathcal{Q}}+\mathcal{N}_{35}^{\mathcal{Q}}$ & & & \\
\hline $\mathcal{Q}_{d W}$ & $" \mathcal{Q}_{d W} "$ & $\mathcal{N}_{33}^{\mathcal{Q}}+\mathcal{N}_{34}^{\mathcal{Q}}+\mathcal{N}_{35}^{\mathcal{Q}}$ & $\mathcal{Q}_{e W}$ & $" \mathcal{Q}_{e W} "$ & $\mathcal{N}_{13}^{\ell}$ \\
\hline $\mathcal{Q}_{u B}$ & $" \mathcal{Q}_{u B} "$ & $\mathcal{N}_{29}^{\mathcal{Q}}+\mathcal{N}_{30}^{\mathcal{Q}}$ & & & \\
\hline $\mathcal{Q}_{d B}$ & $" \mathcal{Q}_{d B} "$ & $\mathcal{N}_{29}^{\mathcal{Q}}+\mathcal{N}_{30}^{\mathcal{Q}}$ & $\mathcal{Q}_{e B}$ & $" \mathcal{Q}_{e B} "$ & $\mathcal{N}_{12}^{\ell}$ \\
\hline $\mathcal{Q}_{q q}^{(1)}$ & $" \mathcal{Q}_{q q}^{(1)} "$ & $R_{9}^{\mathcal{Q}}$ & $\mathcal{Q}_{l l}$ & $" \mathcal{Q}_{l l} "$ & $R_{2}^{\ell}$ \\
\hline $\mathcal{Q}_{q q}^{(3)}$ & $" \mathcal{Q}_{q q}^{(3)} "$ & $R_{12}^{\mathcal{Q}}$ & $\mathcal{Q}_{l q}^{(1)}$ & $" \mathcal{Q}_{l q}^{(1)} "$ & $R_{7}^{\mathcal{Q} \ell}$ \\
\hline $\mathcal{Q}_{u u}$ & 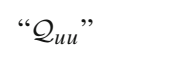 & $R_{13}^{\mathcal{Q}}+R_{14}^{\mathcal{Q}}+R_{15}^{\mathcal{Q}}$ & $\mathcal{Q}_{l q}^{(3)}$ & $" \mathcal{Q}_{l q}^{(3)} "$ & $R_{13}^{\mathcal{Q} \ell}$ \\
\hline $\mathcal{Q}_{d d}$ & $" \mathcal{Q}_{d d} "$ & $R_{13}^{\mathcal{Q}}+R_{14}^{\mathcal{Q}}+R_{15}^{\mathcal{Q}}$ & $\mathcal{Q}_{e e}$ & $" \mathcal{Q}_{e e} "$ & $R_{3}^{\ell}$ \\
\hline $\mathcal{Q}_{u d}^{(1)}$ & $" \mathcal{Q}_{u d}^{(1)} "$ & $R_{13}^{\mathcal{Q}}+R_{15}^{\mathcal{Q}}$ & $\mathcal{Q}_{e u}$ & "Q⿱ $\mathcal{Q}_{\text {eu" }}$ & $R_{8}^{\mathcal{Q} \ell}+R_{10}^{\mathcal{Q} \ell}$ \\
\hline $\mathcal{Q}_{u d}^{(8)}$ & $" \mathcal{Q}_{u d}^{(8)} "$ & $R_{13}^{\mathcal{Q}}+R_{16}^{\mathcal{Q}}+R_{15}^{\mathcal{Q}}$ & $\mathcal{Q}_{e d}$ & $" \mathcal{Q}_{e d} "$ & $R_{8}^{\mathcal{Q} \ell}+R_{10}^{\mathcal{Q} \ell}$ \\
\hline $\mathcal{Q}_{q u}^{(1)}$ & " $\mathcal{Q}_{q u}^{(1)} "$ & $R_{17}^{\mathcal{Q}}+R_{18}^{\mathcal{Q}}$ & $\mathcal{Q}_{l e}$ & $" \mathcal{Q}_{l e} "$ & $R_{6}^{\ell}$ \\
\hline $\mathcal{Q}_{q u}^{(8)}$ & " $\mathcal{Q}_{q u}^{(8)} "$ & $R_{22}^{\mathcal{Q}}+R_{23}^{\mathcal{Q}}$ & $\mathcal{Q}_{l u}$ & "Q⿱中⿰㇀丶㇀ & $R_{14}^{\mathcal{Q} \ell}+R_{18}^{\mathcal{Q} \ell}$ \\
\hline $\mathcal{Q}_{q d}^{(1)}$ & $" \mathcal{Q}_{q d}^{(1)} "$ & $R_{17}^{\mathcal{Q}}+R_{18}^{\mathcal{Q}}$ & $\mathcal{Q}_{l d}$ & $" \mathcal{Q}_{l d} "$ & $R_{14}^{\mathcal{Q} \ell}+R_{18}^{\mathcal{Q} \ell}$ \\
\hline $\mathcal{Q}_{q d}^{(8)}$ & $" \mathcal{Q}_{q d}^{(8)} "$ & $R_{22}^{\mathcal{Q}}+R_{23}^{\mathcal{Q}}$ & $\mathcal{Q}_{q e}$ & $" \mathcal{Q}_{q e} "$ & $R_{15}^{\mathcal{Q} \ell}$ \\
\hline $\mathcal{Q}_{q u q d}^{(1)}$ & "Q $\mathcal{Q}_{\text {quqd }}^{(1)} "$ & $R_{1}^{\mathcal{Q}}+R_{2}^{\mathcal{Q}}$ & $\mathcal{Q}_{l e d q}$ & "Q $\mathcal{Q}_{\text {lelq }} "$ & $R_{21}^{\mathcal{Q} \ell}+R_{22}^{\mathcal{Q} \ell}$ \\
\hline \multirow[t]{2}{*}{$\mathcal{Q}_{q u q d}^{(8)}$} & " $\mathcal{Q}_{\text {quqd }}^{(8)} "$ & $R_{5}^{\mathcal{Q}}+R_{6}^{\mathcal{Q}}$ & $\mathcal{Q}_{\text {lequ }}^{(1)}$ & " $\mathcal{Q}_{\text {lequ }}^{(1)} "$ & $R_{2}^{\mathcal{Q} \ell}+R_{6}^{\mathcal{Q} \ell}$ \\
\hline & & & $\mathcal{Q}_{\text {lequ }}^{(3)}$ & "Q $\mathcal{Q}_{\text {lequ }}^{(3)} "$ & $R_{1}^{\mathcal{Q} \ell}+R_{2}^{\mathcal{Q} \ell}+R_{3}^{\mathcal{Q} \ell}+R_{5}^{\mathcal{Q} \ell}+R_{6}^{\mathcal{Q} \ell}$ \\
\hline
\end{tabular}

ence between these two linear bases (the first two columns in both tables) lies in the choice of two invariants: in Refs. [48,49] the EOMs have been used for removing the fermionic terms corresponding to $\mathcal{Q}_{\varphi l, i i}^{(1)}$ and $\mathcal{Q}_{\varphi l, i i}^{(3)}$ in Ref. [7], replacing them with the bosonic operators $\mathcal{O}_{B}$ and $\mathcal{O}_{W}$. In the HEFT construction, the EOMs have been applied analogously to Refs. [48,49], namely retaining $\mathcal{P}_{B}$ and $\mathcal{P}_{W}$, rather than two leptonic invariants (see Eq. (D.14)).

All the HEFT operators that do not appear in this list have SMEFT counterparts (dubbed also "linear siblings") of dimension larger than six and therefore are not contained in the bases of Refs. [7,48,49].

\section{Phenomenology}

\subsection{Physical parameters definitions}

The phenomenological analysis is carried out in the Zscheme, defined by the following set of observables, that are taken as input parameters:

$\alpha_{S} \quad$ world average [50],

$G_{F}$ extracted from the muon decay rate [50],

$\alpha_{\mathrm{em}}$ extracted from Thomson scattering [50],

$M_{Z}$ extracted from the $Z$ lineshape at LEP I [50],

$M_{h}$ measured at LHC [51]. 
All the other quantities appearing in the Lagrangian will be implicitly interpreted as corresponding to the combinations of experimental inputs as follows:

$$
\begin{aligned}
& e^{2}=4 \pi \alpha_{\mathrm{em}}, \quad \sin ^{2} \theta_{W}=\frac{1}{2}\left(1-\sqrt{1-\frac{4 \pi \alpha_{\mathrm{em}}}{\sqrt{2} G_{F} M_{Z}^{2}}}\right), \\
& v^{2}=\frac{1}{\sqrt{2} G_{F}},\left.\quad\left(g=\frac{e}{\sin \theta_{W}}, \quad g^{\prime}=\frac{e}{\cos \theta_{W}}\right)\right|_{\theta_{W}, e \text { as above }} .
\end{aligned}
$$

The trigonometric functions $\sin \theta_{W}, \cos \theta_{W}$ will be conveniently shortened to $s_{\theta}, c_{\theta}$.

The kinetic terms are made canonical and diagonal with the following field redefinitions:

$$
\begin{aligned}
A_{\mu} \rightarrow & A_{\mu}\left[1+s_{2 \theta} c_{1}+2 s_{\theta}^{2} c_{12}-\frac{1}{2}\left(c_{\theta}^{2} c_{B}+s_{\theta}^{2} c_{W}\right)\right] \\
& +Z_{\mu} 2\left[c_{2 \theta} c_{1}+s_{2 \theta}\left(c_{12}+\frac{c_{B}-c_{W}}{4}\right)\right]+\mathcal{O}\left(c_{i}^{2}\right) \\
Z_{\mu} \rightarrow & Z_{\mu}\left[1-s_{2 \theta} c_{1}+2 c_{\theta}^{2} c_{12}-\frac{1}{2}\left(c_{\theta}^{2} c_{W}+s_{\theta}^{2} c_{B}\right)\right] \\
& +\mathcal{O}\left(c_{i}^{2}\right) \\
W_{\mu}^{+} \rightarrow & W_{\mu}^{+}\left[1-\frac{1}{2} c_{W}\right]+\mathcal{O}\left(c_{i}^{2}\right) .
\end{aligned}
$$

The contributions to the input parameters at first order in the effective coefficients read

$$
\begin{aligned}
& \frac{\delta \alpha_{\mathrm{em}}}{\alpha_{\mathrm{em}}} \simeq 2 s_{2 \theta} c_{1}+4 s_{\theta}^{2} c_{12}-c_{\theta}^{2} c_{B}-s_{\theta}^{2} c_{W}, \\
& \frac{\delta G_{F}}{G_{F}} \simeq-64 \sqrt{2} \pi^{2} \frac{v^{2}}{\Lambda^{2}}\left(r_{2}^{\ell}-r_{5}^{\ell}\right), \\
& \frac{\delta M_{Z}}{M_{Z}} \simeq-c_{T}-s_{2 \theta} c_{1}+2 c_{\theta}^{2} c_{12}-\frac{1}{2}\left(c_{\theta}^{2} c_{W}+s_{\theta}^{2} c_{B}\right), \\
& \frac{\delta M_{h}}{M_{h}} \simeq 0 .
\end{aligned}
$$

The resulting shifts for the $W$ mass and fermion couplings to gauge bosons with respect to their corresponding SM expectations due to these finite renormalisation effects are summarised below:

$\boldsymbol{W}$ mass:

$$
\begin{aligned}
\frac{\Delta M_{W}}{M_{W}}= & \frac{c_{\theta}^{2}}{c_{2 \theta}} c_{T}+\frac{s_{2 \theta}}{c_{2 \theta}} c_{1}-2 c_{12} \\
& +\frac{32 \pi^{2} \sqrt{2} s_{\theta}^{2}}{c_{2 \theta}} \frac{v^{2}}{\Lambda^{2}}\left(r_{2}^{\ell}-r_{5}^{\ell}\right) .
\end{aligned}
$$

\section{Fermionic couplings:}

It is convenient to adopt the following compact notation:

$$
\begin{aligned}
\Delta g_{1} & =c_{T}+32 \pi^{2} \sqrt{2} \frac{v^{2}}{\Lambda^{2}}\left(r_{2}^{\ell}-r_{5}^{\ell}\right), \\
\Delta g_{W} & =\frac{c_{\theta}^{2}}{c_{2 \theta}} c_{T}+t_{2 \theta} c_{1}-2 c_{12}+\frac{32 \pi^{2} \sqrt{2} c_{\theta}^{2}}{c_{2 \theta}} \frac{v^{2}}{\Lambda^{2}}\left(r_{2}^{\ell}-r_{5}^{\ell}\right), \\
\Delta g_{2} & =-s_{\theta}^{2}\left(-\frac{\delta s_{\theta}^{2}}{s_{\theta}^{2}}-\frac{\beta}{t_{\theta}}\right) \\
& =\frac{s_{2 \theta}^{2}}{2 c_{2 \theta}}\left(c_{T}+\frac{2 c_{1}}{s_{2 \theta}}+32 \pi^{2} \sqrt{2} \frac{v^{2}}{\Lambda^{2}}\left(r_{2}^{\ell}-r_{5}^{\ell}\right)\right),
\end{aligned}
$$

where $\Delta g_{1}$ accounts for the renormalisation of $Z_{\mu}, g$ and $c_{\theta}$ in the combination $g Z_{\mu} / c_{\theta} ; \Delta g_{W}$ for the renormalisation of $W_{\mu}$ and $g$ in the combination $g W_{\mu} ; \Delta g_{2}$ for the renormalisation of $s_{\theta}^{2}$ and for the contribution to the $Z$ couplings that comes from the redefinition of the photon field: $A \rightarrow \alpha A+\beta Z$ (see Eq. (3.3)). With this notation, the renormalisation of $Z$ couplings to left-handed and right-handed fermions, $g_{L}^{f}=\left(T_{3}^{f}-s_{\theta}^{2} Q^{f}\right)$ and $g_{R}^{f}=-s_{\theta}^{2} Q^{f}$, and of the $W$ to left-handed fermions can be written as

$\Delta g_{L, R}^{f}=g_{L, R}^{f} \Delta g_{1}+Q^{f} \Delta g_{2} \quad \Delta g_{W}^{f f^{\prime}}=\Delta g_{W}$,

where $Q_{f}$ and $T_{3 f}$ are, respectively, the electric and isospin charges of the fermion $f$, and where the $W$ couplings to left-handed fermions is normalised to 1 in the SM.

The next sections are dedicated to the discussion of the constraints imposed on the operator coefficients considering respectively electroweak precision data, Higgs results from the LHC and the Tevatron, and measurements of the triple gauge-bosons couplings. For the sake of simplicity we will assume fermion universality as well as the absence of new sources of flavour violation.

\subsection{Constraints from EWPD}

After accounting for finite renormalisation effects in the gauge bosons' wavefunctions and couplings as well as for direct contributions to the vertices, 12 operators modify the $Z$ and $W$ gauge-boson couplings to fermions with the same Lorentz structure as the SM and the $W$ mass, which correspondingly lead to linear modifications of the EWPD.

Five operators, $\mathcal{P}_{T}(h), \mathcal{P}_{1}(h), \mathcal{P}_{12}(h), \mathcal{R}_{2}^{\ell}(h), \mathcal{R}_{5}^{\ell}(h)$ give tree level contributions to universal modifications of the couplings and of the $W$ mass, which can be recast in terms of the oblique $S, T, U$ parameters $[52,53]$ and of the shift in the Fermi constant $\Delta G_{F}$. In particular

$$
\begin{aligned}
& \alpha S=-8 s_{\theta} c_{\theta} c_{1}, \quad \alpha T=2 c_{T}, \quad \alpha U=-16 s_{\theta}^{2} c_{12}, \\
& \frac{\delta G_{F}}{G_{F}}=-64 \pi^{2} \sqrt{2} \frac{v^{2}}{\Lambda^{2}}\left(r_{2}^{\ell}-r_{5}^{\ell}\right),
\end{aligned}
$$


so, for example, the correction to the $W$ mass in Eq. (3.5) reads

$$
\frac{\Delta M_{W}}{M_{W}}=\frac{c_{\theta}^{2}}{2 c_{2 \theta}} \alpha T-\frac{1}{4 c_{2 \theta}} \alpha S+\frac{1}{8 s_{\theta}^{2}} \alpha U-\frac{s_{\theta}^{2}}{2 c_{2 \theta}} \frac{\delta G_{F}}{G_{F}} .
$$

The other seven operators, $\mathcal{N}_{1}^{\mathcal{Q}}(h), \mathcal{N}_{2}^{\mathcal{Q}}(h), \mathcal{N}_{5}^{\mathcal{Q}}(h), \mathcal{N}_{6}^{\mathcal{Q}}(h)$, $\mathcal{N}_{7}^{\mathcal{Q}}(h), \mathcal{N}_{8}^{\mathcal{Q}}(h), \mathcal{N}_{2}^{\ell}(h)$, give fermion dependent contributions to the $W$ and $Z$ couplings. Altogether the shifts to the SM $Z$ couplings can be written as

$\Delta g_{L, R}^{f}=g_{L, R}^{f} \Delta g_{1}+Q^{f} \Delta g_{2}+\Delta \tilde{g}_{L, R}^{f}$,

where the finite renormalisation shifts of the fermion couplings in Eq. (3.6) can be rewritten as:

$$
\begin{aligned}
& \Delta g_{1}=\frac{1}{2}\left(\alpha T-\frac{\delta G_{F}}{G_{F}}\right), \\
& \Delta g_{2}=\frac{s_{\theta}^{2}}{c_{2 \theta}}\left(c_{\theta}^{2}\left(\alpha T-\frac{\delta G_{F}}{G_{F}}\right)-\frac{1}{4 s_{\theta}^{2}} \alpha S\right),
\end{aligned}
$$

while the fermion dependent modification of the couplings $\mathrm{read}^{3}$

$$
\begin{array}{ll}
\Delta \tilde{g}_{L}^{u}=n_{1}^{\mathcal{Q}}+2 n_{5}^{\mathcal{Q}}+n_{7}^{\mathcal{Q},}, & \Delta \tilde{g}_{R}^{u}=n_{2}^{\mathcal{Q}}+2 n_{6}^{\mathcal{Q}}+n_{8}^{\mathcal{Q}}, \\
\Delta \tilde{g}_{L}^{d}=-n_{1}^{\mathcal{Q}}+2 n_{5}^{\mathcal{Q}}-n_{7}^{\mathcal{Q},}, & \Delta \tilde{g}_{R}^{d}=-n_{2}^{\mathcal{Q}}+2 n_{6}^{\mathcal{Q}}-n_{8}^{\mathcal{Q}}, \\
\Delta \tilde{g}_{L}^{\nu}=0, & \Delta \tilde{g}_{R}^{v}=0, \\
\Delta \tilde{g}_{L}^{e}=0, & \Delta \tilde{g}_{R}^{e}=2 n_{2}^{\ell} .
\end{array}
$$

The corresponding shifts to the $W$ couplings to left-handed fermions (normalised to 1 in the SM) are

$\Delta g_{W}^{f f^{\prime}}=\Delta g_{W}+\Delta \tilde{g}_{W}^{f f^{\prime}}$,

with the universal shift due to the finite renormalisation defined in Eq. (3.6) given by

$\Delta g_{W}=\frac{\Delta M_{W}}{M_{W}}-\frac{1}{2} \frac{\delta G_{F}}{G_{F}}$,

and the fermion dependent shifts induced by the fermionic operators by

$\Delta \tilde{g}_{W}^{u d}=2 n_{1}^{\mathcal{Q}}-2 n_{7}^{\mathcal{Q}}, \quad \Delta \tilde{g}_{W}^{e v}=0$.

There are two main differences with respect to the corresponding contributions to EWPD obtained assuming a linear realisation of the $S U(2)_{L} \times U(1)_{Y}$ gauge symmetry breaking with operators up to dimension six (see for example Refs. $[54,55])$.

\footnotetext{
$\overline{3}$ One could expect $\Delta \tilde{g}_{L}^{\nu, e}$ to have a similar contributions as $\Delta \tilde{g}_{L}^{u, d}$. This is not the case as the corresponding leptonic operators have been removed from the basis by using the EOMs, as discussed in Eq. (D.14). This choice simplifies the renormalisation procedure as $\Delta \tilde{g}_{L}^{v, e}$ are vanishing.
}

- First, in the SMEFT no contribution to the $U$ parameter is generated at dimension six, while a contribution is generated in the HEFT at NLO, $\mathcal{O}\left(p^{4}\right)$.

- Second, in the linear description and assuming universality, the fermion dependent shifts of the $W$ couplings to fermions are directly determined by those of the $Z$ as there are only five independent dimension- 6 operators entering those vertices with SM Lorentz structure (which can be chosen for example to be $\mathcal{O}_{\phi q}^{(3)}, \mathcal{O}_{\phi q}^{(1)}, \mathcal{O}_{\phi u}, \mathcal{O}_{\phi d}$, $\mathcal{O}_{\phi e}$ in the notation of Ref. [7]). In the chiral description at order $p^{4}$ the fermion dependent contributions come in contrast from the seven operators given above, of which six combinations contribute independently to EWPD.

So altogether 10 combinations of the 12 operator coefficients can be determined by the analysis of EWPD which have been chosen here to be $c_{T}, c_{1}, c_{12},\left(r_{2}^{\ell}-r_{5}^{\ell}\right), n_{1}^{\mathcal{Q}}$, $\left(n_{2}^{\mathcal{Q}}+n_{8}^{\mathcal{Q}}\right), n_{5}^{\mathcal{Q}}, n_{6}^{\mathcal{Q}}, n_{7}^{\mathcal{Q}}$ and $n_{2}^{\ell}$. In order to obtain the corresponding constraints on these 10 parameters a fit including 16 experimental data points is performed. These are $13 \mathrm{Z}$ observables: $\Gamma_{Z}, \sigma_{h}^{0}, P_{\tau}^{\mathrm{pol}}, \sin ^{2} \theta^{\ell}$ eff, $R_{l}^{0}, \mathcal{A}_{l}$ (SLD), $A_{\mathrm{FB}}^{0, l}$, $R_{c}^{0}, R_{b}^{0}, \mathcal{A}_{c}, \mathcal{A}_{b}, A_{\mathrm{FB}}^{0, c}$, and $A_{\mathrm{FB}}^{0, b}$ from SLD/LEP-I [56], plus three $W$ observables: the average of the $W$-boson mass, from [57], the $W$ width, $\Gamma_{W}$, from LEP-II/Tevatron [58], and the leptonic $W$ branching ratio, $B r_{W}^{e v}$, for which the average in Ref. [50] is taken. The correlations among the inputs can be found in Ref. [56] and have been taken into consideration in the analysis. As mentioned above, unlike in the fits to dimension-6 SMEFT operators, the independent experimental information on the $W$ couplings to fermions have been included in the present study: this is done by considering in the fit the leptonic $W$ branching ratio, as it is measured independently of the total $W$ width, which is determined from kinematic distributions. The corresponding predictions for the observables in the analysis in terms of the shifts of the SM couplings defined above are given by

$$
\begin{aligned}
\Delta \Gamma_{Z}= & 2 \Gamma_{Z, \mathrm{SM}}\left(\frac{\sum_{f}\left(g_{L}^{f} \Delta g_{L}^{f}+g_{R}^{f} \Delta g_{R}^{f}\right) N_{C}^{f}}{\sum_{f}\left(\left|g_{L}^{f}\right|^{2}+\left|g_{R}^{f}\right|^{2}\right) N_{C}^{f}}\right) \\
\Delta \sigma_{h}^{0}= & 2 \sigma_{h, \mathrm{SM}}^{0}\left(\frac{\left(g_{L}^{e} \Delta g_{L}^{e}+g_{R}^{e} \Delta g_{R}^{e}\right)}{\left|g_{L}^{e}\right|^{2}+\left|g_{R}^{e}\right|^{2}}\right. \\
& \left.+\frac{\sum_{q}\left(g_{L}^{q} \Delta g_{L}^{q}+g_{R}^{q} \Delta g_{R}^{q}\right)}{\sum_{q}\left(\left|g_{L}^{q}\right|^{2}+\left|g_{R}^{q}\right|^{2}\right)}-\frac{\Delta \Gamma_{Z}}{\Gamma_{Z, \mathrm{SM}}}\right) \\
\Delta R_{l}^{0} \equiv & \Delta\left(\frac{\Gamma_{Z}^{\mathrm{had}}}{\Gamma_{Z}^{l}}\right)=2 R_{l, \mathrm{SM}}^{0}\left(\frac{\sum_{q}\left(g_{L}^{q} \Delta g_{L}^{q}+g_{R}^{q} \Delta g_{R}^{q}\right)}{\sum_{q}\left(\left|g_{L}^{q}\right|^{2}+\left|g_{R}^{q}\right|^{2}\right)}\right. \\
& \left.-\frac{\left(g_{L}^{l} \Delta g_{L}^{l}+g_{R}^{l} \Delta g_{R}^{l}\right)}{\left|g_{L}^{l}\right|^{2}+\left|g_{R}^{l}\right|^{2}}\right)
\end{aligned}
$$




$$
\begin{aligned}
& \Delta R_{q}^{0} \equiv \Delta\left(\frac{\Gamma_{Z}^{q}}{\Gamma_{Z}^{\mathrm{had}}}\right)=2 R_{q, \mathrm{SM}}^{0}\left(\frac{\left(g_{L}^{q} \Delta g_{L}^{q}+g_{R}^{q} \Delta g_{R}^{q}\right)}{\left|g_{L}^{q}\right|^{2}+\left|g_{R}^{q}\right|^{2}}\right. \\
& \left.-\frac{\sum_{q^{\prime}}\left(g_{L}^{q^{\prime}} \Delta g_{L}^{q^{\prime}}+g_{R}^{q^{\prime}} \Delta g_{R}^{q^{\prime}}\right)}{\sum_{q^{\prime}}\left(\left|g_{L}^{q^{\prime}}\right|^{2}+\left|g_{R}^{q^{\prime}}\right|^{2}\right)}\right) \text {, } \\
& \Delta \sin ^{2} \theta_{\mathrm{eff}}^{l}=\sin ^{2} \theta_{\mathrm{eff}, \mathrm{SM}}^{l} \frac{g_{L}^{l}}{g_{L}^{l}-g_{R}^{l}}\left(\frac{\Delta g_{R}^{f}}{g_{R}^{f}}-\frac{\Delta g_{L}^{f}}{g_{L}^{f}}\right), \\
& \Delta \mathcal{A}_{f}=4 \mathcal{A}_{f, \mathrm{SM}} \frac{g_{L}^{f} g_{R}^{f}}{\left|g_{L}^{f}\right|^{4}-\left|g_{R}^{f}\right|^{4}}\left(g_{R}^{f} \Delta g_{L}^{f}-g_{L}^{f} \Delta g_{R}^{f}\right), \\
& \Delta P_{\tau}^{\mathrm{pol}}=\Delta \mathcal{A}_{l}, \\
& \Delta A_{\mathrm{FB}}^{0, f}=A_{\mathrm{FB}, \mathrm{SM}}^{0, f}\left(\frac{\Delta \mathcal{A}_{l}}{\mathcal{A}_{l}}+\frac{\Delta \mathcal{A}_{f}}{\mathcal{A}_{f}}\right), \\
& \Delta \Gamma_{W}=\Gamma_{W, \mathrm{SM}}\left(\frac{4}{3} \Delta g_{W}^{u d}+\frac{2}{3} \Delta g_{W}^{e v}+\Delta M_{W}\right), \\
& \Delta B r_{W}^{e v}=B r_{W, \mathrm{SM}}^{e v}\left(-\frac{4}{3} \Delta g_{W}^{u d}+\frac{4}{3} \Delta g_{W}^{e v}\right) .
\end{aligned}
$$

When performing the fit within the context of the SM the result is $\chi_{\mathrm{EWPD}, \mathrm{SM}}^{2}=18.3$, while when including the 10 new parameters it gets reduced to $\chi_{\text {EWPD,min }}^{2}=6$. The results of the analysis are shown in Fig. 1 which displays the $\Delta \chi_{\text {EWPD }}^{2}$ dependence of the 10 independent operator coefficients. In each panel $\Delta \chi_{\mathrm{EWPD}}^{2}$ is shown after marginalising over the other nine coefficients. The figure shows the corresponding $95 \%$ allowed ranges given in Table 3: the only operator coefficient not compatible with zero at $2 \sigma$ is $n_{2}^{\mathcal{Q}}+n_{8}^{\mathcal{Q}}$, a result driven by the $2.7 \sigma$ discrepancy between the observed $A_{\mathrm{FB}}^{0, b}$ and the SM expectation.

It is interesting to notice that the resulting constraints on the coefficients contributing to $T, U$ and $\delta G_{F}$ are considerably weaker than what one would obtain in the standard three-parameter fits to $S, T, U$. Quantitatively, the results of the 10-parameter analysis performed here give the following $1 \sigma$ ranges for $S, T, U$ and $\delta G_{F}$ :

$S=-0.45 \pm 0.37, \quad T=-0.3 \pm 2.8, \quad U=-0.1 \pm 2.5$,

$\frac{\delta G_{F}}{G_{F}}=(0.08 \pm 2.2) \times 10^{-2}$,

to be compared with the results of the standard threeparameter fit for $S, T, U$ [55],

$S=0.08 \pm 0.1, \quad T=-0.1 \pm 0.12, \quad U=0.0 \pm 0.09$.

While the range for $S$ is only about 4 times broader when including the effects of all the additional operators, the bounds on $T$ and $U$ are weakened by more than a factor 20 . The main reason is that when $\delta G_{F}$ is also included in the analysis cancellations can occur. In particular as can be seen in Eq. (3.9)-(3.11) for

$\alpha T=\frac{\delta G_{F}}{G_{F}}=-\frac{1}{4 s_{\theta}^{2}} \alpha U$

the contributions from $T, U$, and $\delta G_{F}$ cancel both in the $Z$ observables and in $\Delta M_{W}$. Therefore, along this direction in the parameter space, the bounds on these three quantities come from the contribution of $\delta G_{F}$ to $\Gamma_{W}$ and $B r_{W}^{e v}$ in Eq. (3.15), but these observables are less precisely determined.

It is important to notice that this "weakening" arises even if the $n_{i}^{f}$ coefficients, that is all the fermion dependent contributions, but the four-fermionic ones, are set to zero and only the four contributions $c_{1}, c_{T}, c_{12}$ and $r_{2}^{\ell}-r_{5}^{\ell}$ are retained. In this particular case, the result of the fit is

$$
\begin{aligned}
& S=-0.1 \pm 0.1, \quad T=0.43 \pm 2.86, \quad U=-0.3 \pm 2.4 \\
& \frac{\delta G_{F}}{G_{F}}=(-0.26 \pm 2.0) \times 10^{-2}
\end{aligned}
$$

to be compared with Eq. (3.26). On the contrary, in the framework of linear dimension- 6 operators, the condition $U=0$ makes this cancellation not possible, so bounds on the corresponding operator coefficients are generically stronger. In other words, when making the EWPD analysis in the context of HEFT at $\mathcal{O}\left(p^{4}\right)$ the bounds on the operators contributing to $T$ and $U$ are generically weaker by more than one order of magnitude.

The fermionic operators can also lead to modifications of the semileptonic decay amplitudes used to determine the elements of the CKM matrix and to test its unitarity. In particular, $\mathcal{N}_{1}^{\mathcal{Q}}(h), \mathcal{N}_{7}^{\mathcal{Q}}(h), \mathcal{R}_{2}^{\ell}(h), \mathcal{R}_{5}^{\ell}(h), \mathcal{R}_{13}^{\mathcal{Q} \ell}(h)$ induce linear shifts to the corresponding amplitudes (normalised to $G_{F}$ as determined from $\mu$ decay) which can be parameterised as a shift in the effective CKM matrix,

$$
\begin{aligned}
& \Delta V_{\mathrm{CKM} i j} \\
& \quad=V_{\mathrm{CKM}, \mathrm{SM}_{i j}}\left(-64 \pi^{2} \sqrt{2} \frac{v^{2}}{\Lambda^{2}} r_{13}^{\mathcal{Q} \ell}+\Delta \tilde{g}_{W}^{u d}-\frac{\delta G_{F}}{G_{F}}\right),
\end{aligned}
$$

and which can lead to violations of unitarity of the CKM matrix which are strongly constrained. In the case of SMEFT with operators up to dimension six, three operators enter this observable after equivalent application of the EOMs $[54,55]$ (which can be chosen for example to be $\mathcal{O}_{\phi q}^{(3)}, \mathcal{O}_{l l}$, and, $\mathcal{O}_{l q}^{(3)}$ Ref. [7]). From the global analysis in Ref. [50]

$$
\begin{aligned}
\sum_{i}\left|V_{u i}\right|^{2}-1 & =2\left(-64 \pi^{2} \sqrt{2} \frac{v^{2}}{\Lambda^{2}} r_{13}^{\mathcal{Q} \ell}+\Delta \tilde{g}_{W}^{u d}-\frac{\delta G_{F}}{G_{F}}\right) \\
& =(-1 \pm 6) \times 10^{-4} .
\end{aligned}
$$


Fig. 1 Dependence of $\Delta \chi_{\mathrm{EWPD}+\mathrm{CKM}}^{2}\left(=\Delta \chi_{\mathrm{EWPD}}^{2}\right.$ for all but last panel) on the 11 independent operator coefficients as labelled in the figure. In each panel $\Delta \chi_{\text {EWPD }+ \text { CKM }}^{2}$ is shown after marginalising over the other undisplayed parameters
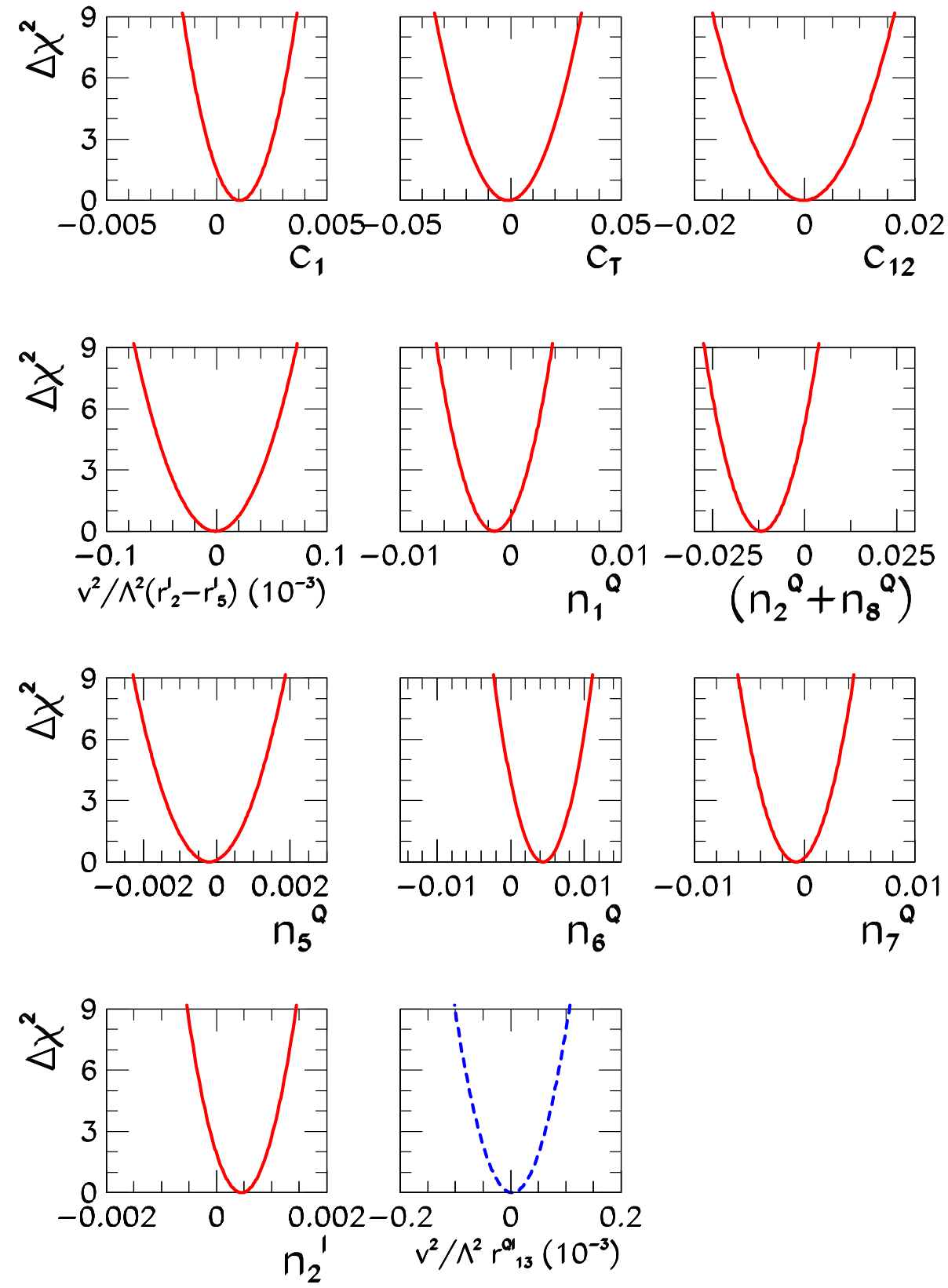

In combination with the analysis of the EWPD, this allows for constraining the coefficient of an 11th operator $\mathcal{R}_{13}^{\mathcal{Q} \ell}(h)$. Adding this data point to the 16 of the EWPD allows one to construct $\chi_{\mathrm{EWPD}+\mathrm{CKM}}^{2}$, which is now a function of 11 parameters (with $\chi_{\mathrm{EWPD}+\mathrm{CKM}, \mathrm{SM}}^{2}=18.4$ and $\chi_{\mathrm{EWPD}+\mathrm{CKM}, \mathrm{min}}^{2}$ $=6)$. The marginalised distributions verify $\Delta \chi_{\mathrm{EWPD}+\mathrm{CKM}}^{2}(x)$ $=\Delta \chi_{\mathrm{EWPD}}^{2}(x)$ for the first 10 parameters, i.e. the inclusion of the CKM unitarity constraint has no impact in the previous analysis as long as $r_{13}^{\mathcal{Q} \ell}$ is allowed to vary free in the fit. The new $\Delta \chi_{\mathrm{EWPD}+\mathrm{CKM}}^{2}\left(r_{13}^{\mathcal{Q} \ell}\right)$ is shown in the curve in the last panel in Fig. 1 and its $95 \%$ CL range is listed in the last row in Table 3.

\subsection{Effects in Higgs physics}

This section is dedicated to the study of the current bounds stemming from the Higgs searches at the LHC. Restricting the analysis to the subset of $C$ and $P$ even operators, ${ }^{4}$ the focus is on those terms that contribute to the trilinear Higgs interactions with fermions and gauge bosons (deviations in the Higgs triple vertex will only become observable in the future). The deviations on Higgs quartic vertices $\left(H V f \bar{f}^{\prime}\right)$ generated by some of the single fermionic current operators

${ }^{4}$ The extension of the analysis to $C P$ odd non-linear operators could be performed after the inclusion of $C P$ sensitive observables; see Ref. [25]. 
Table $395 \%$ allowed ranges for the combinations of operator coefficients entering the EWPD analysis and the CKM unitarity test

\begin{tabular}{ll}
\hline Coupling & $95 \%$ allowed range \\
\hline$c_{1}$ & $(-0.66,2.7) \times 10^{-3}$ \\
$c_{T}$ & $(-0.023,0.021)$ \\
$c_{12}$ & $(-0.011,0.011)$ \\
$\frac{v^{2}}{\Lambda^{2}}\left(r_{2}^{\ell}-r_{5}^{\ell}\right)$ & $(-4.9,4.7) \times 10^{-5}$ \\
$n_{1}^{\mathcal{Q}}$ & $(-4.9,2.0) \times 10^{-3}$ \\
$n_{2}^{\mathcal{Q}}+n_{8}^{\mathcal{Q}}$ & $(-22,-1.5) \times 10^{-3}$ \\
$n_{5}^{\mathcal{Q}}$ & $(-1.6,1.2) \times 10^{-3}$ \\
$n_{6}^{\mathcal{Q}}$ & $(-0.025,8.8) \times 10^{-3}$ \\
$n_{7}^{\mathcal{Q}}$ & $(-4.2,2.7) \times 10^{-3}$ \\
$n_{2}^{\ell}$ & $(-0.2,1.1) \times 10^{-3}$ \\
$\frac{v^{2}}{\Lambda^{2}} r_{13}^{\mathcal{Q} \ell}$ & $(-7.1,6.6) \times 10^{-5}$ \\
\hline
\end{tabular}

have been omitted from this analysis. Those contributions to Higgs physics could also be studied at the LHC [59-61] and, if analysed in combination with gauge-fermion data, they would potentially improve the comparison between linear and non-linear scenarios [60,61]. Nevertheless the generalisation of the analysis with the inclusion of these effects is out of the scope of the present study. The list of operators analysed includes then $\mathcal{P}_{T}(h), \mathcal{P}_{B, G, W}(h)$ and $\mathcal{P}_{1,4,5,12,17}(h)$, in addition to the contributions from $Y_{U}^{(1)}, Y_{D}^{(1)}, Y_{\ell}^{(1)}$ and to the deviations in the GBs kinetic term parameterised by $\Delta a_{C}$. This set can be further reduced considering the strong constraints imposed on $\mathcal{P}_{T, 1,12}(h)$ by the global analysis of EWPD at the $Z$ pole: the impact of these operators on Higgs physics can be safely neglected, given the accuracy at which these observables are currently measured. Moreover, the current Higgs searches are only sensitive to $\mathrm{Hff}$ vertices with $f=t, b, \tau$ (the addition of $\mu$ to the analysis will be straightforward once the sensitivity to this coupling increases). Therefore, only a subset of 10 operators is relevant for the analysis of the available Higgs data. Their contributions to the several Higgs trilinear interactions can be illustrated with the usual HVV phenomenological Lagrangian in the unitary gauge:

$$
\begin{aligned}
\mathcal{L}= & g_{H g g} H G_{\mu \nu}^{a} G^{a \mu \nu}+g_{H \gamma \gamma} H A_{\mu \nu} A^{\mu \nu} \\
& +g_{H Z \gamma}^{(1)} A_{\mu \nu} Z^{\mu} \partial^{v} H+g_{H Z \gamma}^{(2)} H A_{\mu \nu} Z^{\mu \nu} \\
& +g_{H Z Z}^{(1)} Z_{\mu \nu} Z^{\mu} \partial^{v} H+g_{H Z Z}^{(2)} H Z_{\mu \nu} Z^{\mu \nu} \\
& +g_{H Z Z}^{(3)} H Z_{\mu} Z^{\mu} \\
& +g_{H W W}^{(1)}\left(W_{\mu \nu}^{+} W^{-\mu} \partial^{\nu} H+\text { h.c. }\right) \\
& +g_{H W W}^{(2)} H W_{\mu \nu}^{+} W^{-\mu \nu}+g_{H W W}^{(3)} H W_{\mu}^{+} W^{-\mu} \\
& +\sum_{f=\tau, b, t}\left(g_{f} H \bar{f}_{L} f_{R}+\text { h.c. }\right) .
\end{aligned}
$$

The 13 parameters in this Lagrangian can be re-written in terms of the following 10 coefficients ${ }^{5}$ :

$\Delta a_{C}, a_{B}, a_{G}, a_{W}, a_{4}, a_{5}, a_{17}, Y_{t}^{(1)}, Y_{b}^{(1)}, Y_{\tau}^{(1)}$,

and explicitly they read

$g_{H g g}=-\frac{1}{2 v} a_{G}$,

$g_{H Z \gamma}^{(1)}=-\frac{g s_{\theta}}{4 \pi v c_{\theta}}\left(a_{5}+2 \frac{c_{\theta}}{s_{\theta}} a_{4}+2 a_{17}\right)$,

$g_{H Z \gamma}^{(2)}=\frac{s_{\theta} c_{\theta}}{v}\left(a_{B}-a_{W}\right)$,

$g_{H Z Z}^{(1)}=\frac{g}{4 \pi v}\left(2 \frac{s_{\theta}}{c_{\theta}} a_{4}-a_{5}-2 a_{17}\right)$,

$g_{H Z Z}^{(2)}=-\frac{1}{2 v}\left(s_{\theta}^{2} a_{B}+c_{\theta}^{2} a_{W}\right)$,

$g_{H Z Z}^{(3)}=M_{Z}^{2}\left(\sqrt{2} G_{F}\right)^{1 / 2}\left(1+\Delta a_{C}\right)$,

$g_{H \gamma \gamma}=-\frac{1}{2 v}\left(s_{\theta}^{2} a_{W}+c_{\theta}^{2} a_{B}\right)$,

$g_{H W W}^{(1)}=-\frac{g}{4 \pi v} a_{5}, \quad g_{H W W}^{(2)}=\frac{1}{v} a_{W}$,

$g_{H W W}^{(3)}=2 M_{W}^{2}\left(\sqrt{2} G_{F}\right)^{1 / 2}\left(1+\Delta a_{C}\right)$,

$g_{f}=-\frac{Y_{f}^{(1)}}{\sqrt{2}}$.

The anomalous Higgs interactions described by these 10 operators can be studied and constrained in a model independent way by means of a global analysis of all the Higgs experimental measurements that were performed at the LHC during the Run I. This includes not only event rate data in several Higgs production and decay categories, but also some kinematic distributions, that have an interesting phenomenological impact, as shown in the context of SMEFT in Ref. [62-67]. Indeed, they are important for allowing one to obtain finite constraints in the large-dimensional parameter space spanned in the global analysis [62]. Moreover, they make it possible to disentangle the non-SM Lorentz structures from the SM-like shifts.

The global analysis of all Run I Higgs, data using the SFITTER framework [68-72] for the SMEFT $[48,49]$, has been presented in Ref. [62]: in that case, the 13 parameters of the phenomenological Lagrangian in Eq. (3.32) received contributions from nine linear operators. Here, that analysis is extended to account for the 10th coefficient $a_{17}$. All the details regarding the data set and the kinematic distributions analysed, as well as the statistical treatment performed in this log-likelihood analysis follow exactly the description presented in Ref. [62] and will not be repeated here.

The results of the global analysis on the parameters in Eq. (3.33) using the available Higgs data, including all the

\footnotetext{
${ }^{5}$ Notice the implicit redefinitions $a_{i} \equiv c_{i} a_{i}$ for the bosonic operators.
} 
Table 4 Best fit and $95 \%$ CL allowed ranges of the coefficients of the operators contributing to Higgs data $\left(a_{G}\right.$, $a_{W}, a_{B}, a_{4}, a_{5}, a_{17}, \Delta a_{C}, Y_{t}^{(1)}$, $Y_{b}^{(1)}$ and $\left.Y_{\tau}^{(1)}\right)$ and to TGV analyses $\left(c_{2}, c_{3}\right.$ and $\left.c_{W W W}\right)$. $Y_{t}^{(1)}, Y_{b}^{(1)}$ and $Y_{\tau}^{(1)}$ are normalised to the SM expectation

\begin{tabular}{|c|c|c|}
\hline & Best fit & $95 \%$ CL region \\
\hline$a_{G}$ & $\begin{array}{c}-0.0125 \\
-0.0030 \\
0.0029 \\
0.0123 \\
\end{array}$ & $\begin{array}{c}(-0.018,-0.0080) \\
(-0.0054,0.0058) \\
(0.0091,0.017)\end{array}$ \\
\hline$a_{W}$ & -0.017 & $(-0.11,0.088)$ \\
\hline$a_{B}$ & 0.0052 & $(-0.025,0.041)$ \\
\hline$a_{4}$ & 0.041 & $(-0.85,1.1)$ \\
\hline$a_{5}$ & 0.13 & $(-0.81,0.60)$ \\
\hline$\Delta a_{C}$ & -0.13 & $(-0.30,0.23)$ \\
\hline$a_{17}$ & 0.055 & $(-0.52,0.65)$ \\
\hline$Y_{t}^{(1)} / Y_{t}^{(0)}$ & $\begin{array}{r}-1.11 \\
1.31\end{array}$ & $\begin{array}{c}(-1.7,-0.53) \\
(0.56,1.7)\end{array}$ \\
\hline$Y_{b}^{(1)} / Y_{b}^{(0)}$ & $\begin{array}{r}-0.70 \\
0.66 \\
\end{array}$ & $\begin{array}{c}(-1.7,-0.39) \\
(0.35,1,7)\end{array}$ \\
\hline$Y_{\tau}^{(1)} / Y_{\tau}^{(0)}$ & $\begin{array}{l}-0.94 \\
0.82 \\
\end{array}$ & $\begin{array}{c}(-1.37,-0.63) \\
(0.66,1.47)\end{array}$ \\
\hline$c_{2}$ & 0.041 & $(-0.24,0.27)$ \\
\hline$c_{3}$ & 0.15 & $(-0.093,0.39)$ \\
\hline$c_{W W W}$ & 0.006 & $(-0.013,0.018)$ \\
\hline
\end{tabular}

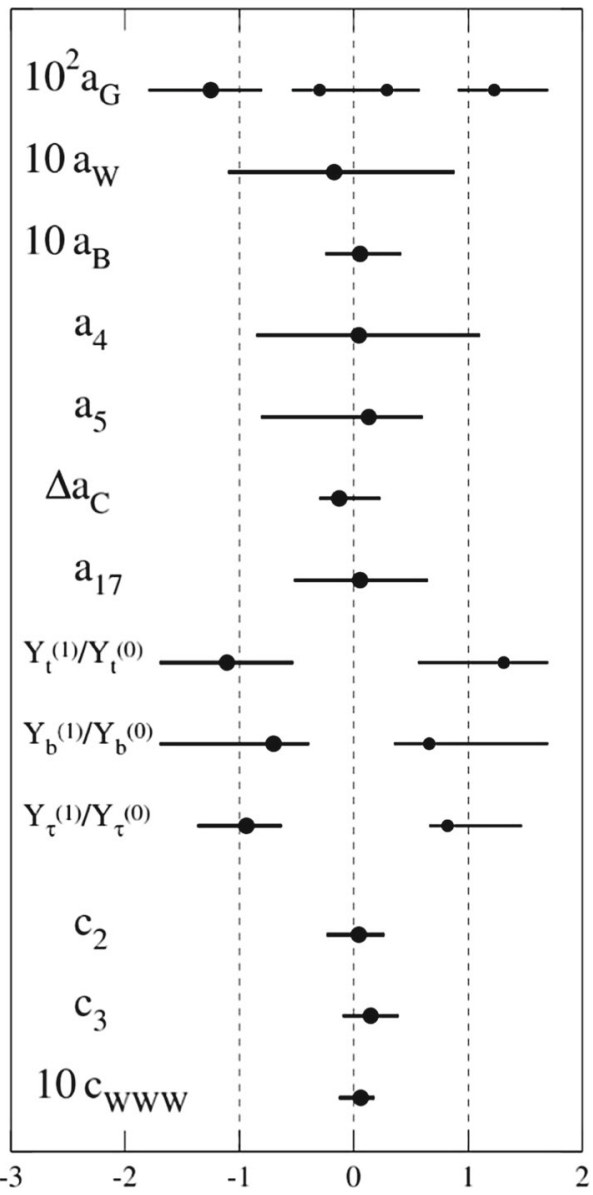

kinematic distributions described in Ref. [62], are reported in Table 4. On the right figure we graphically display the corresponding values where error bars refer to the $95 \% \mathrm{CL}$ allowed ranges, obtained profiling for each coefficient on the other nine parameters that are included in the global analysis. The off-shell $m_{4 \ell}$ distributions, which have been implemented in Ref. [62], are not included here, as their impact in the present analysis is subdominant with respect to the rest of kinematic distributions considered.

The addition of the extra parameter, $a_{17}$, has enlarged the allowed range for all the rest of coefficients contributing to the bosonic Higgs trilinear interactions $\left(a_{4}, a_{5}, a_{W}, a_{B}\right.$ and $\Delta a_{C}$ ) in comparison with the results in Refs. [62,73] (after taking into account the different normalisations used between the two analyses). This was expected given the larger dimensionality of the parameter space analysed here. The new contributions from $\mathcal{P}_{17}(h)$ are consequently strongly correlated to some of the other operators, as illustrated in Fig. 2, where the two-dimensional planes $a_{B}$ vs. $a_{17}$ and $a_{4}$ vs. $a_{17}$ are shown, after profiling on the rest of undisplayed coefficients for each of the panels.

In the present analysis the addition of kinematic distributions is crucial both for closing the allowed regions on all the considered parameters, and for controlling the correlations among the anomalous couplings [62]. To the best of our knowledge, the results derived here present the most complete set of Higgs based constraints on the set of operators of the HEFT Lagrangian. They highlight, in addition, the potential of the EFT expansion to describe and study the Higgs interactions at the LHC.

\subsection{Triple gauge-boson couplings and Higgs interplay}

The study of triple gauge-boson vertices is complementary to the analysis of Higgs physics, and it is fundamental for obtaining a more complete description of the EWSB sector. Focusing again on the $C$ and $P$ even operators and after including the strong constraints from EWPD, only four operators, $\mathcal{P}_{2}(h), \mathcal{P}_{3}(h), \mathcal{P}_{13}(h)$ and $\mathcal{P}_{W W W}(h)$, enter this analysis. ${ }^{6}$ They can give observable deviations from the SM predictions for the triple gauge-boson vertices $W W Z$ and $W W \gamma$. These anomalous contributions can be parameterised

\footnotetext{
${ }^{6}$ An additional operator, $\mathcal{P}_{14}(h)$, generates a $C P$ conserving but $C$ and $P$ violating coupling, whose effects and numerical analysis have been discussed in Refs. $[24,74]$ and also hold here.
} 

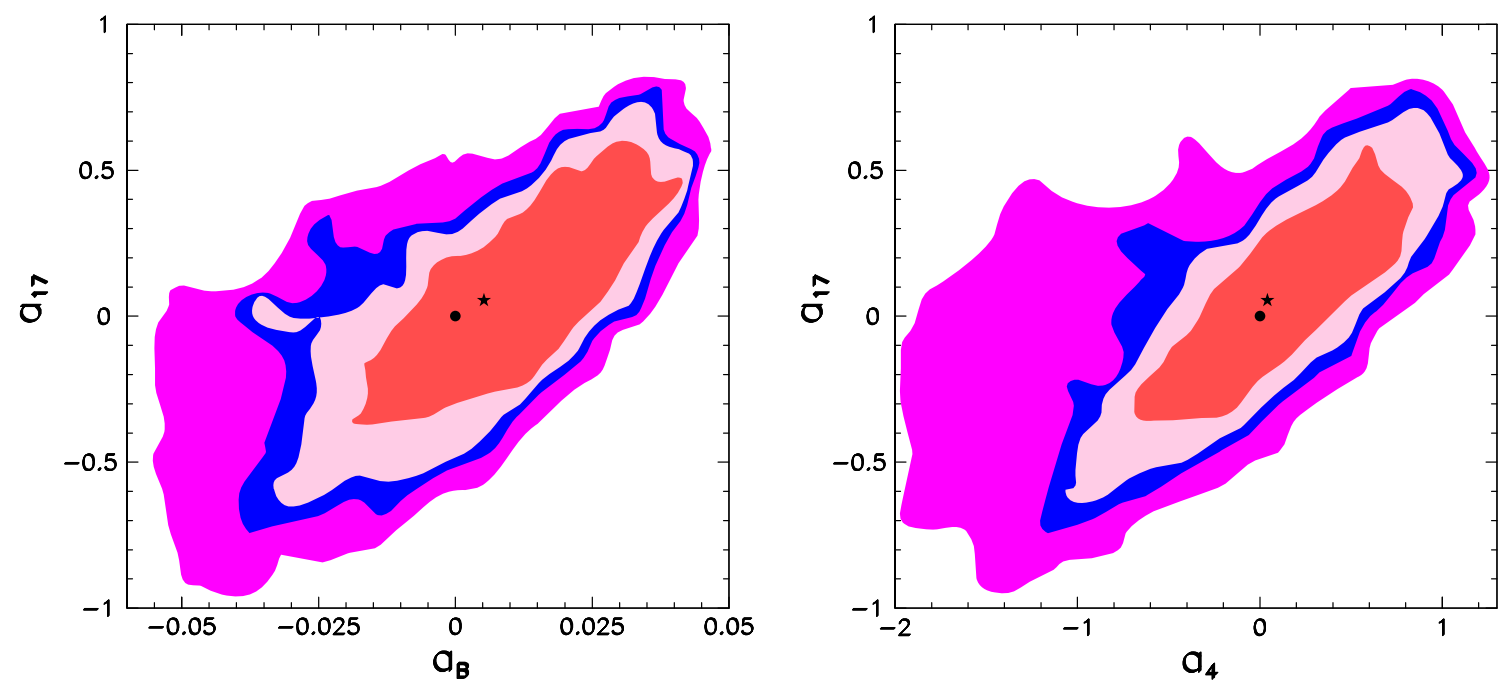

Fig. 2 Results of the global analysis of LHC Higgs run I data, including kinematic distributions, for $\left\{a_{B}, a_{4}, a_{17}\right\}$, profiling on the undisplayed parameters. The colours refers to the different CL regions: from the inner to outer, $68,90,95,99 \% \mathrm{CL}$

in terms of the usual phenomenological TGV Lagrangian presented in Ref. [75]:

$$
\begin{aligned}
\mathcal{L}_{W W V}= & -i g_{W W V}\left\{g_{1}^{V}\left(W_{\mu \nu}^{+} W^{-\mu} V^{\nu}-W_{\mu}^{+} V_{\nu} W^{-\mu \nu}\right)\right. \\
& \left.+\kappa_{V} W_{\mu}^{+} W_{\nu}^{-} V^{\mu \nu}+\frac{\lambda_{V}}{2 m_{W}^{2}} W_{\mu \nu}^{+} W^{-\nu \rho} V_{\rho}^{\mu}\right\},
\end{aligned}
$$

with deviations from the SM predictions, $g_{1}^{Z}=\kappa_{Z}=\kappa_{\gamma}=$ $1, \lambda_{\gamma}=\lambda_{Z}=1$,

$$
\begin{aligned}
& \Delta g_{1}^{Z}=g_{1}^{Z}-1 \equiv \frac{g}{4 \pi c_{\theta}^{2}} c_{3}, \\
& \Delta \kappa_{Z}=\kappa_{Z}-1 \equiv \frac{g}{4 \pi}\left(c_{3}+2 c_{13}-2 t_{\theta} c_{2}\right), \\
& \Delta \kappa_{\gamma}=\kappa_{\gamma}-1 \equiv \frac{g}{4 \pi}\left(c_{3}+2 c_{13}+2 \frac{c_{2}}{t_{\theta}}\right), \\
& \lambda_{\gamma}=\lambda_{Z} \equiv \frac{6 \pi g v^{2}}{\Lambda^{2}} c_{W W W} .
\end{aligned}
$$

Electromagnetic gauge invariance enforces $g_{1}^{\gamma}=1$, both in the SM and in the presence of the new operators. In Eq. (3.35), $V \equiv\{\gamma, Z\}, g_{W W \gamma}=e, g_{W W Z}=g \cos \theta_{W}$, and $W_{\mu \nu}^{ \pm}$and $V_{\mu \nu}$ refer exclusively to the kinetic part of the gauge field strengths.

The combination of all the most sensitive searches for anomalous TGV deviations in $W V$ diboson production has been performed in Ref. [76], presenting the results obtained in the SMEFT framework. These results show that at present the most stringent constraints on the anomalous TGV are set by the LHC Run I searches, whose combined sensitivity has clearly surpassed that of LEP. Even more relevant is the fact that, while the LHC Higgs data and gauge-boson pair production searches are able to separately set stringent constraints on the HEFT operators, the combined study of the two sets of data could be used to improve the understanding of the nature of the Higgs boson state, as already emphasised in Ref. [24].

In brief, three CP even SMEFT operators with $d=6$ can lead to sizeable corrections to the TGV vertices after considering all bounds from EWPD [47-49,62,76]:

$$
\begin{aligned}
& \mathcal{O}_{W}=\frac{i g}{2}\left(D_{\mu} \Phi\right)^{\dagger} W^{\mu \nu}\left(D_{\nu} \Phi\right), \\
& \mathcal{O}_{B}=\frac{i g^{\prime}}{2}\left(D_{\mu} \Phi\right)^{\dagger} B^{\mu \nu}\left(D_{\nu} \Phi\right), \\
& \mathcal{O}_{W W W}=-\frac{i g^{3}}{8} \operatorname{Tr}\left(W_{\mu \nu} W^{\nu \rho} W_{\rho}^{\mu}\right),
\end{aligned}
$$

where the notation of the original papers has been kept.

As pointed out in Ref. [24], comparing the interactions generated by these three operators with those induced by the relevant operators in the HEFT basis, one finds two differences: (i) for the TGV phenomenology $\mathcal{O}_{\mathcal{W}}$ and $\mathcal{O}_{B}$ give corrections to the vertices equivalent to those induced by $\mathcal{P}_{2}(h)$ and $\mathcal{P}_{3}(h)$, while for the HVV couplings their effects are equivalent to those of $\mathcal{P}_{4}(h)$ and $\mathcal{P}_{5}(h)$; (ii) the $\mathcal{O}\left(p^{4}\right)$ chiral operator $\mathcal{P}_{13}(h)$ has no equivalent in the linear expansion at dimension 6.

In other words, (i) implies that, as is well known from the pre-LHC times [77], and recently emphasised in some of the post-Higgs discovery analyses $[49,66,78]$, the operators $\mathcal{O}_{W}$ and $\mathcal{O}_{B}$ lead at the same time to anomalous contributions to both Higgs physics and TGV anomalous measurements. Thus, any deviation generated by them should be correlated in data from both sectors, and consequently the combined analysis of Higgs data and TGV measurements becomes 


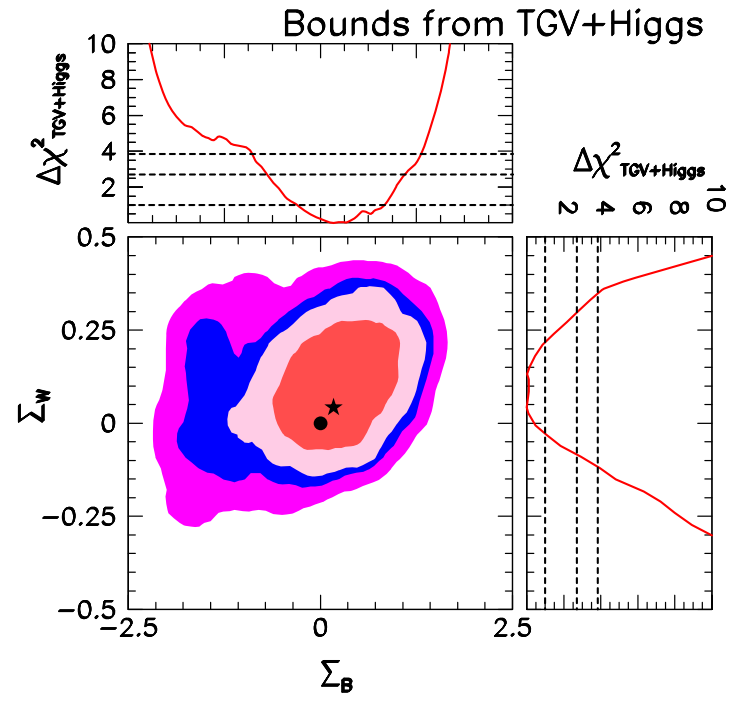

Fig. 3 Present bounds on $\Sigma_{B}, \Sigma_{W}, \Delta_{B}$ and $\Delta_{W}$ (see the text for the details of their definition) as obtained from the most recent combined global analysis of Higgs and TGV data. The rest of the undisplayed parameters spanned in the global analysis

mandatory in order to obtain constraints as strong as possible on their coefficients [76]. Conversely, in the HEFT case, the anomalous TGV deviations induced by $\mathcal{O}_{W}$ and $\mathcal{O}_{B}$ are generated by $\mathcal{P}_{2}(h)$ and $\mathcal{P}_{3}(h)$, while their effects on Higgs physics originate from $\mathcal{P}_{4}(h)$ and $\mathcal{P}_{5}(h)$. Therefore, deviations in TGV and in Higgs physics could remain completely uncorrelated in the HEFT context [24]. This means that the nature of the Higgs boson can be directly probed by testing the presence of this (de)-correlated pattern of interactions in the event of an anomalous observation in any of the two sectors.

To illustrate the present status of such comparison, a global analysis of the data available both on the Higgs interactions and on the searches for anomalous TGV has been performed. The analysis spans the 10 coefficients relevant for Higgs physics in the HEFT scenario; see Eq. (3.33), together with the three parameters relevant for the TGV sector, which have an equivalent in the SMEFT Lagrangian, $c_{2}, c_{3}$ and $c_{W W W}$ (i.e. setting $c_{13}$ to zero). ${ }^{7}$

In what respects the TGV analysis, the simulation of the relevant distributions and the statistical fit follow those of Ref. [76]. The best fit values and 95\% CL intervals obtained for $c_{2}, c_{3}$ and $c_{W W W}$ are quoted for completeness in Table 4. As can be seen comparing the results in Table 4 with Table 4 of Ref. [24], derived considering only the LEP based TGV bounds on $c_{2}$ and $c_{3}$, the new combination of LHC Run I

\footnotetext{
7 Notice that the operator belonging to the SMEFT expansion which contains the same interactions described by $\mathcal{P}_{13}(h)$, also called "linear sibling", arises only at $d=8$.
}

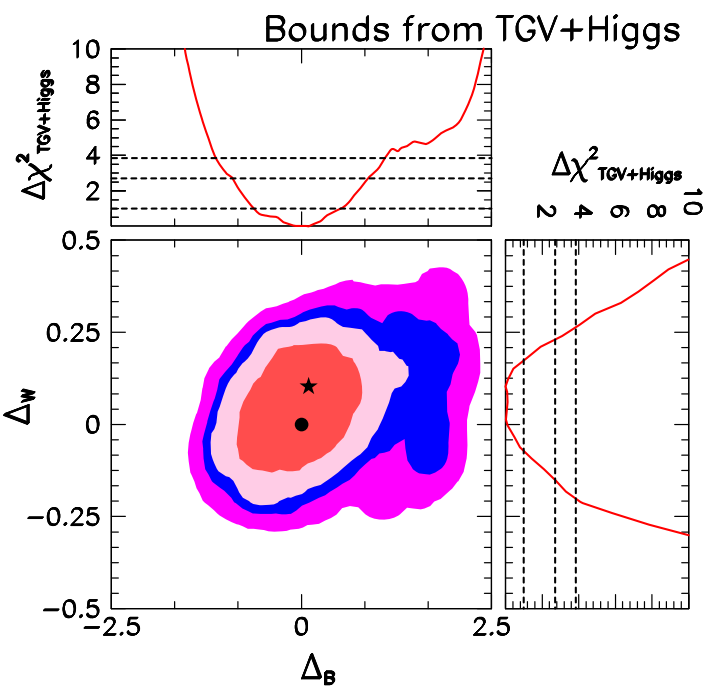

$\left(\Delta a_{C}, a_{B}, a_{G}, a_{W},, a_{17}, Y_{t}^{(1)}, Y_{b}^{(1)}, Y_{\tau}^{(1)}\right.$ and $\left.c_{W W W}\right)$ have been profiled. The black dots signal the $(0,0)$ point, while the stars signal the current best fit point obtained in the analysis

searches is able to improve substantially the constraints on $\mathcal{P}_{2}(h)$ and $\mathcal{P}_{3}(h)$.

It was already shown in Ref. [24] that four specific combinations of the coefficients $\mathcal{P}_{2}(h), \mathcal{P}_{3}(h), \mathcal{P}_{4}(h)$ and $\mathcal{P}_{5}(h)$ are meaningful for illustrating the Higgs+TGV results: ${ }^{8}$

$$
\begin{aligned}
\Sigma_{B} & \equiv \frac{1}{\pi g t_{\theta}}\left(2 c_{2}+a_{4}\right), \quad \Sigma_{W} \equiv \frac{1}{2 \pi g}\left(2 c_{3}-a_{5}\right), \\
\Delta_{B} & \equiv \frac{1}{\pi g t_{\theta}}\left(2 c_{2}-a_{4}\right), \quad \Delta_{W} \equiv \frac{1}{2 \pi g}\left(2 c_{3}+a_{5}\right) .
\end{aligned}
$$

These four parameters were defined in such a way that, at $d=6$ order in the SMEFT expansion, the two $\Delta$ 's are zero because of gauge invariance and of the doublet nature of the Higgs, $\Delta_{B}=\Delta_{W}=0$. On the other hand, the operators $\mathcal{O}_{W}$ and $\mathcal{O}_{B}$ contribute to the $\Sigma$ 's leading to $\Sigma_{B}=v^{2} \frac{f_{B}}{\Lambda^{2}}$ and $\Sigma_{W}=v^{2} \frac{f_{W}}{\Lambda^{2}}$, being $f_{i}$ the associated Wilson coefficients. In contrast, the HEFT operators could generate independent modifications to each of these four variables. Figure 3 shows the current status of the bounds on the two relevant planes of coefficients after taking into consideration all the Higgs measurements included in the presented Higgs global analysis (based on Ref. [62]), together with the most recent combination of TGV searches presented in the previous subsection (based on Ref. [76]).

As described in Ref. [24], in the left panel of Fig. 3 the $(0,0)$ point corresponds to no deviation from the SM, while

\footnotetext{
${ }^{8}$ For the sake of comparison with Ref. [24], the four combinations have been defined to be quantitatively equivalent to those in Ref. [24], in spite of the different normalisation for the $c_{i}$ and $a_{i}$ coefficients used here.
} 
in the right one it represents the limit in which TGV and HVV couplings show a SMEFT-like correlation. Therefore, any deviation from $(0,0)$ in the left panel would indicate BSM physics irrespective of the nature of the EWSB realisation, while a similar departure in the right panel would disfavour a linear EWSB. As the $\Delta$ 's and the $\Sigma$ 's are orthogonal combinations of parameters, the two panels of Fig. 3 are in principle independent of each other. In particular, deviations from $(0,0)$ may occur arbitrarily in only one plane or in both at the same time.

The constraints of $\Sigma_{B}, \Sigma_{W}, \Delta_{B}$ and $\Delta_{W}$ shown in Fig. 3 present a significant improvement with respect to the bounds previously shown in Fig. 2 of Ref. [24]. The reason for such a sizeable improvement relies on two key points. First, the strength of the derived results is increased by the inclusion of the more complete set of run I LHC Higgs event rate measurement and by the addition of relevant kinematic distributions, that are sensitive to the anomalous SM Lorentz structures generated by $a_{3}$ and $a_{5}$ [62]. Second, the combination of the significant LHC Run I diboson production analysis as described in Ref. [76] also has a huge impact in the analysis. The combination of these two ameliorations enhances significantly the accuracy of the combined results shown in Fig. 3, in spite of the larger dimensionality of the parameter space considered in the present study with respect to the global analysis in Ref. [24].

\section{Higher order operators and expansion validity}

An important issue for numerical analyses performed in an EFT approach is that of establishing whether the EFT description is valid at the typical energies of the processes considered. The task is particularly relevant when collider data is included in the analysis, as the corresponding measurements are typically taken at energies significantly higher than the EW scale.

In general, the validity of the expansion can be discussed studying the impact of operators which belong to different expansion orders. In the context of the SMEFT, this is tantamount to analysing operators with dimension $d>6$. As discussed in Refs. [41,79-82], this analysis sets different constraints on the cut-off of the theory, depending on the observables and of the operators considered: the strongest bounds are associated to observables that receive contributions from $d=8$ operators with a larger number of derivatives, as they induce a strong energy-dependence.

Similar general considerations also apply to the HEFT. However, in this case the discussion is complicated by the simultaneous presence of several characteristic scales and, consequently, of multiple expansion parameters. Although the only physical scales of the HEFT are $\Lambda$ and $v$, as explained in Sect. 1, it is useful to keep momentarily the scale $f(\Lambda \leq$
$4 \pi f)$ as an independent quantity. The limit $f \rightarrow v$ will be discussed later on.

In realistic composite Higgs models, that can be considered as a benchmark for understanding the role played by each scale, $v, f$ and $\Lambda$ enter the low-energy Lagrangian in three different combinations: $v / f=\sqrt{\xi}, 1 / 4 \pi \leq f / \Lambda \leq 1$, and $E / \Lambda$, where $E$ is the characteristic energy scale of a given observable. As shown in Ref. [38], cross sections of physical processes only depend on scale suppressions: the generic expression, adopting the NDA normalisation of Eq. (2.7), is given by

$\sigma \sim \frac{\pi(4 \pi)^{2}}{E^{2}}\left(\frac{E^{2}}{\Lambda^{2}}\right)^{-N_{\Lambda}}$

where $\left(-N_{\Lambda}\right)$ is the number of powers of $\Lambda$ that suppress an interaction term. The NDA master formula takes automatically care of all the $4 \pi$ factors appearing in the cross-section (see Ref. [38] for further details and for generalisations), so that $\left(-N_{\Lambda}\right)$ actually counts both powers of $\Lambda$ and of $f$ indifferently. As a result, the only quantities that can be considered as proper suppression factors are $\sqrt{\xi}$ and $E / \Lambda$. The physical relevance of a given cross-sections is basically determined by its dependence on these two parameters.

While the dependence on $1 / \Lambda$ is explicit in HEFT operators, it is less trivial to trace that on $\sqrt{\xi}=v / f$. To this aim, it is useful to recall (see Sect. 1) that $f$ is the scale associated to both the SM GBs and the Higgs and, as such, it is always hidden inside the GB matrix $\mathbf{U}(x)$ and the generic Higgs functions $\mathcal{F}(h)$. The dependence on $f$ can be made explicit expanding these structures:

$\mathbf{U}=1+2 i \frac{\sigma_{a} \pi^{a}}{f}+\cdots, \quad \mathcal{F}(h)=1+2 a \frac{h}{f}+\cdots$.

Within $\mathbf{V}_{\mu}$ and upon going to unitary gauge, the powers on $1 / f$ are converted into factors of $\sqrt{\xi}$. This is due to the fact that, in the kind of scenarios considered here, $\xi$ represents a fine-tuning that necessarily weights insertions of longitudinal components of the gauge bosons [38]. This indeed occurs in composite Higgs models (see Refs. [30,31]), where analogous conclusions are found to hold also for $\partial_{\mu} \mathcal{F}(h)$.

It is worth noticing that, while $\mathbf{U}(x)$ and $\mathcal{F}(h)$, considered globally, are adimensional quantities, their expansions contain terms with different canonical dimensions that come suppressed by powers of $f$. As a result, the leading terms of $\mathbf{V}_{\mu}$ and $\partial_{\mu} \mathcal{F}(h)$, obtained applying one derivative to the series of Eq. (4.2), have canonical dimension two: one dimension being associated to the derivative and the other to the first non-vanishing term in the expansion of either $\mathbf{U}$ or $\mathcal{F}(h)$. This observation can be generalised introducing the primary dimension $d_{p}$, defined in Ref. [38] as the canonical dimension of the leading term in the expansion of a given object. For fundamental elements, such as derivatives, gauge fields and fermions, the primary dimension coincides with the tra- 
Table 5 Different HEFT building blocks and their primary dimensions. The two last columns report the suppression factors associated to each object

\begin{tabular}{llll}
\hline Building block & $d_{p}$ & Factors of $\xi$ & Factors of $p / \Lambda$ \\
\hline $\mathbf{U}(x)$ & 0 & 1 & 1 \\
$\mathcal{F}(h)$ & 0 & 1 & 1 \\
$\partial_{\mu}$ & 1 & 1 & $(p / \Lambda)$ \\
$\psi$ & $3 / 2$ & 1 & $(p / \Lambda)^{3 / 2}$ \\
$X_{\mu \nu}$ & 2 & 1 & $(p / \Lambda)^{2}$ \\
$\mathbf{V}_{\mu}$ & 2 & $\sqrt{\xi}$ & $(p / \Lambda)$ \\
$\partial_{\mu} \mathcal{F}(h)$ & 2 & $\sqrt{\xi}$ & $(p / \Lambda)$ \\
\hline
\end{tabular}

ditional canonical dimension. Table 5 contains a summary of the primary dimensions for the building blocks used in the construction of the HEFT Lagrangian, together with the associated suppression factors. It follows from the discussion above that a term suppressed by $\xi^{\alpha / 2}(p / \Lambda)^{\beta}$ must have $d_{p}=\alpha+\beta$.

With the information provided by Table 5, it is easy to infer the dependences for all the HEFT operators, that can be thus organised in a two-parameter expansion as indicated, schematically, in Table 6 . The colours discriminate between two sets of operators: the structures reported in the cyan boxes correspond to the NLO Lagrangian considered in this work; the structures in the white cells, instead, are customarily considered as higher order terms, but their impact may be compa- rable to that of the NLO terms for sufficiently high energies. Depending on the observables considered, it may be necessary to include (part of) the second set of operators into the phenomenological analysis (see also Ref. [83]), even if this would mean working with a ill-defined basis from a renormalisation point of view. This should not be seen as a concern, as, even considering a complete, non-redundant basis at NNLO, only the subcategories listed in Table 6 would be physically relevant. Effects due to operator mixing under the renormalisation group running are also expected to be completely negligible at the experimental sensitivities foreseen for the near future.

In the limit $f \rightarrow v$, the dependence on $\xi$ does not represent a suppression anymore and the physical impact of an operator is determined only by the factors of $p / \Lambda$. In this case, one recovers a pure chiral expansion, which is organised "horizontally" in the representation of Table 6.

On the contrary, in the limit $p / \Lambda \simeq \sqrt{\xi}$, all the operators with the same $d_{p}$ are equally suppressed and therefore one recovers, altogether, the linear expansion organised in canonical (or primary) dimensions. In this case, all the operators in the white boxes of Table 6 should be considered. This condition is for instance fulfilled for $\Lambda=10 \mathrm{TeV}$ and $E \simeq 1 \mathrm{TeV}$, which is within the range of energies that are relevant for processes to be observed at LHC13.

The introduction of the primary dimension, i.e. of a counting on explicit and implicit scale suppressions, allows one to link the particular structure of an operator to the strength
Table 6 HEFT operators distributed according to their $\xi$ and $p / \Lambda$ suppressing factors. A schematic notation has been adopted for categorising the operators based on the building blocks they contain. The terms appearing in the cyan boxes correspond to the NLO operators listed in the previous sections. The other terms refer to operators that usually belong to higher Lagrangian orders, but that can have an impact similar to that of the NLO ones for sufficiently high energies. EOMs have been employed to remove redundant structures

\begin{tabular}{|c|c|c|c|c|c|}
\hline$\xi^{2}$ & $\begin{array}{l}(\partial \mathcal{F})^{2}(\mathbf{V})^{2} \\
(\mathbf{V})^{4} \\
(\partial \mathcal{F})^{4}\end{array}$ & & & & \\
\hline$\xi^{3 / 2}$ & & & & & \\
\hline$\xi$ & $\begin{array}{l}\left(\mathbf{V}^{2}\right)(X)^{2} \\
(\partial \mathcal{F})(\mathbf{V})(X)\end{array}$ & $\begin{array}{l}(\partial \mathcal{F})(\mathbf{V})(\bar{\psi} \psi) \\
(\mathbf{V})^{2}(\bar{\psi} \psi) \\
(\partial \mathcal{F})^{2}(\bar{\psi} \psi)\end{array}$ & $\begin{array}{l}(X)^{2}(\mathbf{V})^{2} \\
(\partial \mathcal{F})(\mathbf{V})(X)^{2} \\
(\partial \mathcal{F})^{2}(X)^{2}\end{array}$ & & \\
\hline$\sqrt{\xi}$ & $(\bar{\psi} \psi)(\mathbf{V})$ & & $\begin{array}{l}(\mathbf{V})(X)(\bar{\psi} \psi) \\
(\partial \mathcal{F})(X)(\bar{\psi} \psi)\end{array}$ & $\begin{array}{l}(\mathbf{V})(\bar{\psi} \psi)^{2} \\
(\partial \mathcal{F})(\bar{\psi} \psi)^{2}\end{array}$ & \\
\hline 1 & $(X)^{2}$ & $(X)(\bar{\psi} \psi)$ & $\begin{array}{l}(\bar{\psi} \psi)^{2} \\
(X)^{3}\end{array}$ & $(X)^{2}(\bar{\psi} \psi)$ & $\begin{array}{l}(X)(\bar{\psi} \psi)^{2} \\
(X)^{4}\end{array}$ \\
\hline & 1 & $\left(\frac{p}{\Lambda}\right)$ & $\left(\frac{p}{\Lambda}\right)^{2}$ & $\left(\frac{p}{\Lambda}\right)^{3}$ & $\left(\frac{p}{\Lambda}\right)^{4}$ \\
\hline
\end{tabular}


of a physical signal in terms of cross sections. Indeed, if an observable receives contributions from a single operator, then the corresponding cross section is uniquely determined by the primary dimension of that operator, according to Eq. (4.1). As a consequence, the $d_{p}$ is a useful phenomenological tool to indicate whether the strength of an observable, that receive contributions only from operators belonging to higher expansion orders, is expected to be of the same order or more suppressed with respect to the other processes already considered in the phenomenological analysis.

An interesting application of the primary dimension is that if the $d_{p}$ of an HEFT operator is smaller than the canonical dimension of the corresponding linear sibling, then the processes described by these operators represent smoking guns to test the linearity of the EWSB realisation. This is the case of the operator $\mathcal{P}_{14}(h)$ discussed in Ref. [38]: it induces an anomalous TGV, commonly called $g_{5}^{Z}$, that is expected to be strongly suppressed in the SMEFT description, but not in the HEFT one.

\section{Conclusions}

The complete effective Lagrangian for a non-linear realisation of the EWSB (shortened into HEFT) has been presented. It provides the most general description of the Higgs couplings and it can be used for investigating a large spectrum of distinct theories, ranging from the SM to technicolour constructions, including composite Higgs realisations and dilaton-like frameworks. In contrast with the effective Lagrangian for a linearly realised EWSB (also SMEFT), in which the Higgs belongs to an exact $S U(2)_{L}$ doublet, in the HEFT the physical Higgs is assigned to a singlet representation of the EW group and it is treated as an object independent of the Goldstone bosons' matrix.

Assuming invariance under the Lorentz and SM gauge symmetries, as well as the conservation of baryon and lepton numbers, the complete chiral basis at the next to leading order contains a total number of 148 independent, flavour universal terms. When extending the SM spectrum to include three right-handed neutrinos, 40 more operators enrich the basis. The generalisation to arbitrary flavour contractions is straightforward.

Conversely, the SMEFT basis up to $d=6$ consists of only 59 flavour universal terms, in absence of right-handed neutrinos. The different number of operators and of building blocks used for the construction of the two bases lead to fundamental differences between the SMEFT and the HEFT. The possibility of distinguishing between them has been discussed performing a global fit including all the available data from colliders, including EWPD, Higgs and TGV measurements taken at the LHC Run I. The main outcomes are summarised in the following points:
- The Electroweak precision data analysis together with the study of the CKM matrix unitarity allows one to constrain 11 parameters of the HEFT Lagrangian. The corresponding value of the $\chi^{2}$ at the minimum is 6 . This can be compared with the corresponding analysis within the SM, whose $\chi^{2}$ is 18.4 .

- The results for the $S, T$ and $U$ parameters are significantly different from the standard analysis in the SMEFT with operators up to dimension 6 , due to the presence of extra free parameters: the allowed range for $S$ is about 4 times broader, while the bounds on $T$ and $U$ are about 20 times weaker.

- The analysis of Higgs data depends on a total of 10 parameters, with one bosonic operator more compared to the same analysis in the SMEFT case at dimension six. Although the final results are quite similar to those obtained for the SMEFT, the addition of the extra parameter broadens the allowed range for the remaining nine coefficients, as expected.

- The interplay between triple gauge-boson vertices and Higgs couplings provides an interesting way of investigating the nature of EWSB. Although this analysis is not conclusive yet due to the limited sensitivity on the observables considered, the introduction of kinematic distributions is seen to improve considerably the results. Would the accuracy of Higgs measurements improve significantly in the future, this kind of analysis may reveal signatures of non-linearity in the Higgs sector.

- It has been underlined that with the increase in energy at colliders, it may be necessary to consider several operators that, in spite of being usually considered as higher order effects, may have a non-negligible phenomenological impact. The list of the relevant structures has been given in Table 6.

In summary, this work extends the chiral basis of Refs. $[24,25]$ with the introduction of fermionic operators. Moreover, the analysis presented here updates and extends that contained in Ref. [24] with the inclusion of more recent collider data and of fermionic observables. A strategy for disentangling the nature of the EWSB has been discussed, based on the presence of new anomalous signals and of decorrelations among observables. It has also been discussed how the phenomenological analysis should be modified when higher energy data is kept into account, specifying the relevant operator structures that should be added to the basis in this case. The analysis presented here represents the first phenomenological study performed with the complete HEFT Lagrangian and it could be taken as a reference for dedicated experimental analyses aimed at shedding light on the Electroweak symmetry breaking sector and the Higgs nature. 
Acknowledgments We thank M. B. Gavela for useful discussions during the development of the project, as well as Anja Butter, Tilman Plehn and Michael Rauch for their crucial assistance with both the Higgs and the TGV analyses. We also thank E.E. Jenkins, A.V. Manohar and M. Trott for interesting comments on the manuscript. I.B. research was supported by an ESR contract of the EU network FP7 ITN INVISIBLES (Marie Curie Actions, PITN-GA-2011-289442). M.C.G$\mathrm{G}$ is supported by USA-NSF grant PHY-13-16617, by grants 2014SGR-104 and by FPA2013-46570 and consolider-ingenio 2010 program CSD-2008-0037. L.M. acknowledge partial support of CiCYT through the project FPA2012-31880 and of the Spanish MINECO's "Centro de Excelencia Severo Ochoa" Programme under grant SEV2012-0249. M.C.G-G and L.M. acknowledge partial support by FP7 ITN INVISIBLES (PITN-GA-2011-289442), FP10 ITN ELUSIVES (H2020-MSCA-ITN-2015-674896) and INVISIBLES-PLUS (H2020MSCA-RISE-2015-690575)

Open Access This article is distributed under the terms of the Creative Commons Attribution 4.0 International License (http://creativecomm ons.org/licenses/by/4.0/), which permits unrestricted use, distribution, and reproduction in any medium, provided you give appropriate credit to the original author(s) and the source, provide a link to the Creative Commons license, and indicate if changes were made.

Funded by SCOAP ${ }^{3}$.

\section{A Additional operators in the presence of RH neutrinos}

Adding right-handed neutrinos to the spectrum amounts to declaring a non-zero upper component for the $L_{R}$ doublet, which shall be defined as $L_{R}=\left(N_{R}, E_{R}\right)^{T}$. Consequently, the lepton Yukawa matrix in the LO Lagrangian Eq. (2.3) has to be generalised to account for the masses and interactions of the neutrinos with the Higgs

$\mathcal{Y}_{L}(h) \equiv \operatorname{diag}\left(\sum_{n} Y_{v}^{(n)} \frac{h^{n}}{v^{n}}, \sum_{n} Y_{\ell}^{(n)} \frac{h^{n}}{v^{n}}\right)$.

In addition, the fermionic basis presented in Sect. 2.3 must be enlarged in order to account for the increased number of possible invariants, as follows:

$$
\begin{aligned}
\Delta \mathscr{L}_{2 F}= & \sum_{j=15}^{17} n_{j}^{\ell} \mathcal{N}_{j}^{\ell}+\sum_{j=18}^{28} \frac{1}{\Lambda}\left(n_{j}^{\ell}+i \tilde{n}_{j}^{\ell}\right) \mathcal{N}_{j}^{\ell} \\
& +\sum_{j=29}^{31} \frac{4 \pi}{\Lambda}\left(n_{j}^{\ell}+i \tilde{n}_{j}^{\ell}\right) \mathcal{N}_{j}^{\ell}, \\
\Delta \mathscr{L}_{4 F}= & \frac{(4 \pi)^{2}}{\Lambda^{2}}\left[\sum_{j=8}^{10}\left(r_{j}^{\ell}+i \tilde{r}_{j}^{\ell}\right) R_{j}^{\ell}+\sum_{j=1}^{15} r_{j}^{\ell} R_{j}^{\ell}\right. \\
& \left.+\sum_{j=24}^{29}\left(r_{j}^{\mathcal{Q} \ell}+i \tilde{r}_{j}^{\mathcal{Q} \ell}\right) R_{j}^{\mathcal{Q} \ell}+\sum_{j=30}^{38} r_{j}^{\mathcal{Q} \ell} R_{j}^{\mathcal{Q} \ell}\right] .
\end{aligned}
$$

The complete list of additional operators is provided in this appendix.
Single leptonic current operators

With one derivative

$$
\begin{aligned}
\mathcal{N}_{15}^{\ell}(h) & \equiv i \bar{L}_{R} \gamma_{\mu} \mathbf{U}^{\dagger} \mathbf{V}^{\mu} \mathbf{U} L_{R} \mathcal{F}, \\
\text { CP } \quad \mathcal{N}_{16}^{\ell}(h) & \equiv \bar{L}_{R} \gamma_{\mu} \mathbf{U}^{\dagger}\left[\mathbf{V}^{\mu}, \mathbf{T}\right] \mathbf{U} L_{R} \mathcal{F}, \\
\mathcal{N}_{17}^{\ell}(h) & \equiv i \bar{L}_{R} \gamma_{\mu} \mathbf{U}^{\dagger} \mathbf{T} \mathbf{V}^{\mu} \mathbf{T} \mathbf{U} L_{R} \mathcal{F} .
\end{aligned}
$$

With two derivatives

$\mathcal{N}_{18}^{\ell}(h) \equiv \bar{L}_{L} \mathbf{T U} L_{R} \partial_{\mu} \mathcal{F} \partial^{\mu} \mathcal{F}^{\prime}$,

$\mathcal{N}_{19}^{\ell}(h) \equiv \bar{L}_{L} \mathbf{V}_{\mu} \mathbf{U} L_{R} \partial^{\mu} \mathcal{F}$,

$\mathcal{N}_{20}^{\ell}(h) \equiv \bar{L}_{L} \mathbf{T} \mathbf{V}_{\mu} \mathbf{T U} L_{R} \partial^{\mu} \mathcal{F}$,

$\mathcal{N}_{21}^{\ell}(h) \equiv \bar{L}_{L} \mathbf{V}_{\mu} \mathbf{V}^{\mu} \mathbf{T U} L_{R} \mathcal{F}$,

$\mathcal{N}_{22}^{\ell}(h) \equiv \bar{L}_{L} \mathbf{T V} \mathbf{T V}^{\mu} \mathbf{T U} L_{R} \mathcal{F}$,

$\mathcal{N}_{23}^{\ell}(h) \equiv \bar{L}_{L} \mathbf{V}_{\mu} \mathbf{T} \mathbf{V}^{\mu} \mathbf{U} L_{R} \mathcal{F}$,

$\mathcal{N}_{24}^{\ell}(h) \equiv \bar{L}_{L} \mathbf{V}_{\mu} \mathbf{T V}^{\mu} \mathbf{T U} L_{R} \mathcal{F}$,

$\mathcal{N}_{25}^{\ell}(h) \equiv \bar{L}_{L} \sigma^{\mu \nu} \mathbf{V}_{\mu} \mathbf{U} L_{R} \partial_{\nu} \mathcal{F}$,

$\mathcal{N}_{26}^{\ell}(h) \equiv \bar{L}_{L} \sigma^{\mu \nu} \mathbf{T V} \mathbf{V}_{\mu} \mathbf{T U} L_{R} \partial_{\nu} \mathcal{F}$,

$\mathcal{N}_{27}^{\ell}(h) \equiv \bar{L}_{L} \sigma^{\mu \nu} \mathbf{V}_{\mu} \mathbf{T V} \mathbf{V}_{\nu} \mathbf{T U} L_{R} \mathcal{F}$,

$\mathcal{N}_{28}^{\ell}(h) \equiv \bar{L}_{L} \sigma^{\mu v}\left[\mathbf{V}_{\mu}, \mathbf{V}_{\nu}\right] \mathbf{T U} L_{R} \mathcal{F}$,

$\mathcal{N}_{29}^{\ell}(h) \equiv i g^{\prime} \bar{L}_{L} \sigma^{\mu \nu} \mathbf{T U} L_{R} B_{\mu \nu} \mathcal{F}$,

$\mathcal{N}_{30}^{\ell}(h) \equiv i g \bar{L}_{L} \sigma^{\mu \nu}\left\{W_{\mu \nu}, \mathbf{T}\right\} \mathbf{U} L_{R} \mathcal{F}$,

$\mathcal{N}_{31}^{\ell}(h) \equiv i g \bar{L}_{L} \sigma^{\mu \nu} \mathbf{T} W_{\mu \nu} \mathbf{T U} L_{R} \mathcal{F}$.

\section{Four-fermion operators}

Additional operators with four leptons:

$R_{8}^{\ell}(h) \equiv\left(\bar{L}_{L} \sigma^{i} \mathbf{U} L_{R}\right)\left(\bar{L}_{L} \sigma^{i} \mathbf{U} L_{R}\right) \mathcal{F}$,

$R_{9}^{\ell}(h) \equiv\left(\bar{L}_{L} \mathbf{U} L_{R}\right)\left(\bar{L}_{L} \mathbf{T U} L_{R}\right) \mathcal{F}$,

$R_{10}^{\ell}(h) \equiv\left(\bar{L}_{L} \mathbf{T U} L_{R}\right)\left(\bar{L}_{L} \mathbf{T U} L_{R}\right) \mathcal{F}$,

$R_{11}^{\ell}(h) \equiv\left(\bar{L}_{R} \gamma_{\mu} L_{R}\right)\left(\bar{L}_{R} \gamma^{\mu} \mathbf{U}^{\dagger} \mathbf{T} \mathbf{U} L_{R}\right) \mathcal{F}$,

$R_{12}^{\ell}(h) \equiv\left(\bar{L}_{R} \gamma_{\mu} \mathbf{U}^{\dagger} \mathbf{T U} L_{R}\right)\left(\bar{L}_{R} \gamma^{\mu} \mathbf{U}^{\dagger} \mathbf{T U} L_{R}\right) \mathcal{F}$,

$R_{13}^{\ell}(h) \equiv\left(\bar{L}_{L} \gamma_{\mu} L_{L}\right)\left(\bar{L}_{R} \gamma^{\mu} \mathbf{U}^{\dagger} \mathbf{T} \mathbf{U} L_{R}\right) \mathcal{F}$,

$R_{14}^{\ell}(h) \equiv\left(\bar{L}_{L} \gamma_{\mu} \mathbf{T} L_{L}\right)\left(\bar{L}_{R} \gamma^{\mu} \mathbf{U}^{\dagger} \mathbf{T} \mathbf{U} L_{R}\right) \mathcal{F}$,

$R_{15}^{\ell}(h) \equiv\left(\bar{L}_{L} \gamma_{\mu} \sigma^{i} L_{L}\right)\left(\bar{L}_{R} \gamma^{\mu} \mathbf{U}^{\dagger} \sigma^{i} \mathbf{U} L_{R}\right) \mathcal{F}$.

Additional mixed operators with two quarks and two leptons

$R_{24}^{\mathcal{Q} \ell}(h) \equiv\left(\bar{L}_{L} \mathbf{U} Q_{R}\right)\left(\bar{Q}_{L} \mathbf{T U} L_{R}\right) \mathcal{F}$,

$R_{25}^{\mathcal{Q} \ell}(h) \equiv\left(\bar{L}_{L} \mathbf{T U} L_{R}\right)\left(\bar{Q}_{L} \mathbf{U} Q_{R}\right) \mathcal{F}$,

$R_{26}^{\mathcal{Q} \ell}(h) \equiv\left(\bar{L}_{L} \mathbf{T U} L_{R}\right)\left(\bar{Q}_{L} \mathbf{T U} Q_{R}\right) \mathcal{F}$,

$R_{27}^{\mathcal{Q} \ell}(h) \equiv\left(\bar{L}_{L} \mathbf{T U} Q_{R}\right)\left(\bar{Q}_{L} \mathbf{T U} L_{R}\right) \mathcal{F}$,

$R_{28}^{\mathcal{Q} \ell}(h) \equiv\left(\bar{L}_{L} \sigma^{i} \mathbf{T U} L_{R}\right)\left(\bar{Q}_{L} \sigma^{i} \mathbf{U} Q_{R}\right) \mathcal{F}$,

$R_{29}^{\mathcal{Q} \ell}(h) \equiv\left(\bar{L}_{L} \sigma^{i} \mathbf{T U} Q_{R}\right)\left(\bar{Q}_{L} \sigma^{i} \mathbf{U} L_{R}\right) \mathcal{F}$,

$R_{30}^{\mathcal{Q} \ell}(h) \equiv\left(\bar{L}_{R} \gamma_{\mu} \mathbf{U}^{\dagger} \mathbf{T} \mathbf{U} L_{R}\right)\left(\bar{Q}_{R} \gamma^{\mu} Q_{R}\right) \mathcal{F}$,

$R_{31}^{\mathcal{Q} \ell}(h) \equiv\left(\bar{L}_{R} \gamma_{\mu} \mathbf{U}^{\dagger} \mathbf{T} \mathbf{U} L_{R}\right)\left(\bar{Q}_{R} \gamma^{\mu} \mathbf{U}^{\dagger} \mathbf{T} \mathbf{U} Q_{R}\right) \mathcal{F}$, 
$R_{32}^{\mathcal{Q} \ell}(h) \equiv\left(\bar{L}_{R} \gamma_{\mu} \mathbf{U}^{\dagger} \sigma^{j} \mathbf{U} L_{R}\right)\left(\bar{Q}_{R} \gamma^{\mu} \mathbf{U}^{\dagger} \sigma^{j} \mathbf{U} Q_{R}\right) \mathcal{F}$,

$R_{33}^{\mathcal{Q} \ell}(h) \equiv\left(\bar{Q}_{L} \gamma_{\mu} Q_{L}\right)\left(\bar{L}_{R} \gamma^{\mu} \mathbf{U}^{\dagger} \mathbf{T} \mathbf{U} L_{R}\right) \mathcal{F}$,

$R_{34}^{\mathcal{Q} \ell}(h) \equiv\left(\bar{Q}_{L} \gamma_{\mu} \mathbf{T} Q_{L}\right)\left(\bar{L}_{R} \gamma^{\mu} \mathbf{U}^{\dagger} \mathbf{T} \mathbf{U} L_{R}\right) \mathcal{F}$,

$R_{35}^{\mathcal{Q} \ell}(h) \equiv\left(\bar{Q}_{L} \gamma_{\mu} \sigma^{j} Q_{L}\right)\left(\bar{L}_{R} \gamma^{\mu} \mathbf{U}^{\dagger} \sigma^{j} \mathbf{U} L_{R}\right) \mathcal{F}$,

$R_{36}^{\mathcal{Q} \ell}(h) \equiv\left(\bar{Q}_{L} \gamma^{\mu} L_{L}\right)\left(\bar{L}_{R} \gamma_{\mu} \mathbf{U}^{\dagger} \mathbf{T U} Q_{R}\right) \mathcal{F}$,

$R_{37}^{\mathcal{Q} \ell}(h) \equiv\left(\bar{Q}_{L} \gamma_{\mu} \mathbf{T} L_{L}\right)\left(\bar{L}_{R} \gamma^{\mu} \mathbf{U}^{\dagger} \mathbf{T} \mathbf{U} Q_{R}\right) \mathcal{F}$,

$R_{38}^{\mathcal{Q} \ell}(h) \equiv\left(\bar{Q}_{L} \gamma^{\mu} \sigma^{j} \mathbf{T} L_{L}\right)\left(\bar{L}_{R} \gamma_{\mu} \mathbf{U}^{\dagger} \sigma^{j} \mathbf{U} Q_{R}\right) \mathcal{F}$

\section{B Removal of $\mathcal{F}(h) F(h)$ from the Higgs and fermions kinetic terms}

All the kinetic terms in the LO Lagrangian, Eq. (2.3), are canonically normalised, despite the fact that the singlet nature of the $h$ field in principle allows one to couple them to a function $\mathcal{F}(h)$. In the case of the gauge-boson kinetic term, the absence of a Higgs-dependence is justified in the assumption that the transverse components of the gauge fields do not interact with the Higgs sector at LO. On the other hand, in the cases of the Higgs and of the fermions' kinetic terms, the dependence $\mathcal{F}(h)$ is completely redundant, as it can be removed via a field redefinition (analogously to what was done in Ref. [84]. See also Ref. [26]). This appendix provides more details about this redefinition.

The coupling of the fermionic kinetic term to a generic Higgs function would be of the form

$\frac{i}{2}(\bar{\psi} \not D \psi-\bar{\psi} \overleftarrow{D} \psi)\left(1+\mathcal{F}_{\psi}(h)\right)$

where $\psi=\{Q, L\}$ and

$\mathcal{F}_{\psi}(h)=c_{\psi}+2 a_{\psi} \frac{h}{v}+b_{\psi} \frac{h^{2}}{v^{2}}+\cdots$

The dependence of $\mathcal{F}_{\psi}(h)$ can therefore be removed via the redefinition

$\psi \rightarrow \psi^{\prime}\left[1+\mathcal{F}_{\psi}(h)\right]^{-1 / 2}$.

As this substitution is applied to the whole Lagrangian, it induces a modification of all the couplings between fermionic and Higgs fields, which can be reabsorbed in redefinitions of the functions $\mathcal{F}_{i}(h)$, which accompany fermionic operators. In particular, this is also true for the LO Yukawa couplings, as they are accompanied by arbitrary polynomials $\mathcal{Y}_{Q, L}(h)$. In conclusion, the insertion of a function $\mathcal{F}_{\psi}(h)$ in the fermionic kinetic term is redundant in the LO Lagrangian of Eq. (2.3).

The Higgs kinetic term may also be written as

$\frac{1}{2} \partial_{\mu} h \partial^{\mu} h\left(1+\mathcal{F}_{H}(h)\right)$,

with

$\mathcal{F}_{H}(h)=c_{H}+2 a_{H} \frac{h}{v}+b_{H} \frac{h^{2}}{v^{2}}+\cdots$
In this case, the $\mathcal{F}_{H}(h)$ function can be removed by the field redefinition

$h^{\prime} \rightarrow \int_{0}^{h} \sqrt{1+\mathcal{F}_{H}(s)} \mathrm{d} s ;$

in fact

$\frac{1}{2} \partial_{\mu} h^{\prime} \partial^{\mu} h^{\prime}=\frac{1}{2}\left[\partial_{\mu} h \sqrt{1+\mathcal{F}_{H}(h)}\right]^{2}=\mathcal{P}_{H}(h)$.

Although this redefinition looks quite involved, it clearly induces modifications of all the Higgs couplings in the Lagrangian. As these are always described by arbitrary coefficients, the redefinition (B.6) can be entirely reabsorbed into redefinitions of the functions $\mathcal{F}_{i}(H)$, which appear in the Lagrangian. As seen for the case of $\mathcal{F}_{\psi}(h)$ above, the presence of $\mathcal{F}_{H}(h)$ in the Higgs kinetic term is redundant within the LO Lagrangian chosen in Eq. (2.3).

\section{B.1 A practical example}

In order to give a practical illustration, one can consider a specific function

$\mathcal{F}_{H}(h)=2 a_{H} \frac{h}{v}$.

Then the equation

$h^{\prime}=\int_{0}^{h} \sqrt{1+2 a_{H} s / v} \mathrm{~d} s=\frac{v}{3 a_{H}}\left[\left(\frac{2 a_{H} h}{v}+1\right)^{3 / 2}-1\right]$

can be solved analytically, obtaining

$h=\frac{v}{2 a_{H}}\left[\left(\frac{3 a_{H}}{v} h^{\prime}+1\right)^{2 / 3}-1\right]$.

Plugging this result into $\mathcal{F}_{C}(h)$ and re-expanding in $h^{\prime} / v$, it gives $^{9}$ :

$\mathcal{F}_{C}(h)=1+2 a_{C} \frac{h^{\prime}}{v}+\left(b_{C}-a_{C} a_{H}\right)\left(\frac{h^{\prime}}{v}\right)^{2}$,

so that the impact of the redefinition can be entirely reabsorbed defining primed coefficients,

$a_{C}^{\prime}=a_{C}, \quad b_{C}^{\prime}=b_{C}-a_{C} a_{H}$.

An analogous redefinition allows one to reabsorb inside the function $\mathcal{Y}_{\psi}^{(n)}$ the effects on the Yukawa interactions.

\footnotetext{
${ }^{9}$ In this computation $a_{H}>0$ is assumed. For negative values the third roots give some complications.
} 


\section{Construction of the fermionic basis}

This appendix provides additional information about the construction of the fermionic basis specifying, in particular, the relation between the structures present in the operators presented in Sect. 2.3 and those that have been removed. In the following, generic fermion fields are denoted by $\psi=\{Q, L\}$ while $\Gamma$ stands for an arbitrary $S U(2)$ structure, combination of the blocks $\left\{\mathbf{T}, \mathbf{V}_{\mu}, D_{\mu}, \sigma^{j}\right\}$. The Lorentz contractions are always explicited and, whenever they are not specified, chiralities are arbitrary. The correspondence between classes of operators is indicated schematically by an arrow $(\rightarrow)$; signs and numerical coefficients are not specified in these relations.

\section{C.1 Useful identities}

A list of useful identities is provided below. Since the building blocks $A=\left\{\mathbf{T}, \mathbf{V}_{\mu}, \mathbf{D}_{\mu} \mathbf{V}^{\mu}\right\}$ are traceless, they can be generically rewritten as $A=\frac{1}{2} \operatorname{Tr}\left[A \sigma^{a}\right] \sigma^{a}$. This yields the relations:

$$
\begin{aligned}
{\left[\mathbf{T}, \mathbf{V}_{\mu}\right] } & =\frac{i}{2} \varepsilon^{i j k} \operatorname{Tr}\left(\mathbf{T} \sigma^{i}\right) \operatorname{Tr}\left(\mathbf{V}_{\mu} \sigma^{j}\right) \sigma^{k}, \\
\left\{\mathbf{T}, \mathbf{V}_{\mu}\right\} & =\operatorname{Tr}\left(\mathbf{T} \mathbf{V}_{\mu}\right) 1, \\
\mathbf{T} \mathbf{V}_{\mu} \mathbf{T} & =\frac{1}{2}\left[\operatorname{Tr}\left(\mathbf{T} \mathbf{V}_{\mu}\right) \operatorname{Tr}\left(\mathbf{T} \sigma^{i}\right)-\operatorname{Tr}\left(\mathbf{V}_{\mu} \sigma^{i}\right)\right] \sigma^{i} \\
& =\mathbf{T} \operatorname{Tr}\left(\mathbf{T} \mathbf{V}_{\mu}\right)-\mathbf{V}_{\mu} .
\end{aligned}
$$

The properties of the $S U(2)$ generators additionally lead to the following identities:

$\mathbf{T} \mathbf{V}_{\mu} \mathbf{V}^{\mu}=\mathbf{V}_{\mu} \mathbf{V}^{\mu} \mathbf{T}$

$\mathbf{T V}_{[\mu} \mathbf{T} \mathbf{V}_{\nu]}=\mathbf{V}_{[\mu} \mathbf{T} \mathbf{V}_{\nu]} \mathbf{T}$,

$\mathbf{T} \mathbf{V}_{[\mu} \mathbf{T V} \mathbf{V}_{\nu]} \mathbf{T}=\mathbf{V}_{[\mu} \mathbf{T} \mathbf{V}_{\nu]}$,

$\mathbf{T}\left[\mathbf{V}_{\mu}, \mathbf{V}_{\nu}\right]=-\left[\mathbf{V}_{\mu}, \mathbf{V}_{\nu}\right] \mathbf{T}-2 \mathbf{V}_{[\mu} \mathbf{T} \mathbf{V}_{\nu]}$.

The transformation properties of $\mathbf{T}$ and $\mathbf{V}_{\mu}$ ensure

$\mathbf{D}_{\mu} \mathbf{T}=\left[\mathbf{V}_{\mu}, \mathbf{T}\right]$,

$\mathbf{V}_{\mu \nu}=\mathbf{D}_{\mu} \mathbf{V}_{\nu}-\mathbf{D}_{\nu} \mathbf{V}_{\mu}=i g W_{\mu \nu}-i g^{\prime} B_{\mu \nu} / 2+\left[\mathbf{V}_{\mu}, \mathbf{V}_{\nu}\right]$

The Fierz identities for chiral (anticommuting) fields have been employed for the reduction of the four-fermion basis

$$
\begin{aligned}
&\left(\bar{A}_{L} B_{R}\right)\left(\bar{C}_{L} D_{R}\right)=- \frac{1}{2}\left(\bar{A}_{L} D_{R}\right)\left(\bar{C}_{L} B_{R}\right) \\
&-\frac{1}{8}\left(\bar{A}_{L} \sigma^{\mu \nu} D_{R}\right)\left(\bar{C}_{L} \sigma_{\mu \nu} B_{R}\right), \\
&\left(\bar{A}_{L} B_{R}\right)\left(\bar{C}_{R} D_{L}\right)=-\frac{1}{2}\left(\bar{A}_{L} \gamma_{\mu} D_{L}\right)\left(\bar{C}_{R} \gamma^{\mu} B_{R}\right), \\
&\left(\bar{A}_{L} \gamma_{\mu} B_{L}\right)\left(\bar{C}_{L} \gamma^{\mu} D_{L}\right)=\left(\bar{A}_{L} \gamma_{\mu} D_{L}\right)\left(\bar{C}_{L} \gamma^{\mu} B_{L}\right), \\
&\left(\bar{A}_{R} \gamma_{\mu} B_{R}\right)\left(\bar{C}_{R} \gamma^{\mu} D_{R}\right)=\left(\bar{A}_{R} \gamma_{\mu} D_{R}\right)\left(\bar{C}_{R} \gamma^{\mu} B_{R}\right) .
\end{aligned}
$$

Whenever they are applied to $S U$ (2) doublets (and $S U$ (3) triplets), these identities must be applied together with the completeness relations for the generators of $S U(2)$ (and of $S U(3))$,

$\sigma_{i j}^{a} \sigma_{m n}^{a}=2 \delta_{i n} \delta_{m j}-\delta_{i j} \delta_{m n}$,

$\lambda_{i j}^{A} \lambda_{m n}^{A}=2 \delta_{i n} \delta_{m j}-\frac{2}{3} \delta_{i j} \delta_{m n}$,

in order to recover the correct gauge contractions. For example, combining Eq. (C.8) with Eqs. (C.11) and (C.12), the scalar identity for quark doublets reads

$$
\begin{aligned}
\left(\bar{Q}_{1 L} Q_{2 R}\right)\left(\bar{Q}_{3 R} Q_{4 L}\right) & \\
= & -\frac{1}{12}\left(\bar{Q}_{1 L} \gamma_{\mu} Q_{4 L}\right)\left(\bar{Q}_{3 R} \gamma^{\mu} Q_{2 R}\right) \\
& -\frac{1}{12}\left(\bar{Q}_{1 L} \gamma_{\mu} \sigma^{k} Q_{4 L}\right)\left(\bar{Q}_{3 R} \gamma^{\mu} \sigma^{k} Q_{2 R}\right) \\
& -\frac{1}{8}\left(\bar{Q}_{1 L} \gamma_{\mu} \lambda^{A} Q_{4 L}\right)\left(\bar{Q}_{3 R} \gamma^{\mu} \lambda^{A} Q_{2 R}\right) \\
& -\frac{1}{8}\left(\bar{Q}_{1 L} \gamma_{\mu} \lambda^{A} \sigma^{k} Q_{4 L}\right)\left(\bar{Q}_{3 R} \gamma^{\mu} \lambda^{A} \sigma^{k} Q_{2 R}\right) .
\end{aligned}
$$

\section{C.2 Construction of $\Delta \mathscr{L}_{2 F}$}

- Since for traceless matrices $\operatorname{Tr}(A B) 1=\{A, B\}$, the operators of the type $\bar{\psi} \gamma_{\mu} \psi \operatorname{Tr}\left(\Gamma_{1} \Gamma_{2}\right) \mathcal{F}$ with $\Gamma_{i}=$ $\left\{\mathbf{T}, \mathbf{V}_{\mu}, \mathbf{D}_{\mu} \mathbf{V}_{\nu}\right\}$ are always equivalent to the bilinears $\bar{\psi} \gamma_{\mu}\left\{\Gamma_{1}, \Gamma_{2}\right\} \psi \mathcal{F}$.

- Bilinears with a derivative on the fermion field and a vector current (e.g. $\left.\left(D_{\mu} \bar{\psi}\right) \gamma^{\mu} X \psi\right)$ can been removed via integration by parts and application of the EOMs (see Appendix D).

- Operators with a derivative on the fermion field but with no gamma matrices (of the type $\bar{\psi} \Gamma^{\mu} D_{\mu} \psi$ ) are removed using the relation $g^{\mu \nu}=\left\{\gamma^{\mu}, \gamma^{\nu}\right\} / 2$, integration by parts and the EOMs:

$$
\begin{aligned}
& \bar{\psi} \Gamma_{\mu} D^{\mu} \psi=\bar{\psi} g_{\mu \nu} \Gamma^{\mu} D^{\nu} \psi=\bar{\psi} \bar{\nabla}(\not D \psi) \\
& +\left.\bar{\psi} \gamma^{v}\right|^{\prime}\left(D_{v} \psi\right) \\
& =\bar{\psi} \not{W}(\not D \psi)-(\bar{\psi} \overleftarrow{I D}) \bigvee \psi-\bar{\psi}(\not D \not) \psi
\end{aligned}
$$

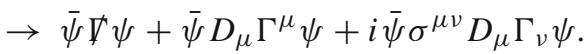

- Bilinears with the structure $\gamma^{\mu} \gamma^{\nu} \mathbf{D}_{\mu} \mathbf{V}_{\nu}$ can be reduced to a combination of dipole operators (containing field strengths), terms with the structure $\sigma^{\mu \nu} \mathbf{V}_{\mu} \mathbf{V}_{\nu}$ and bilinears with the direct contraction $\mathbf{D}_{\mu} \mathbf{V}^{\mu}$. In fact:

$$
\begin{aligned}
\gamma^{\mu} \gamma^{v} \mathbf{D}_{\mu} \mathbf{V}_{\nu} & =\left(g^{\mu \nu}-i \sigma^{\mu \nu}\right) \mathbf{D}_{\mu} \mathbf{V}_{\nu} \\
& =\mathbf{D}_{\mu} \mathbf{V}^{\mu}-\frac{i}{2} \sigma^{\mu \nu} \mathbf{V}_{\mu \nu}
\end{aligned}
$$


where Eq. (C.6) shall be applied on the latter term. The former can also be removed using the EOMs.

- the commutator $\left[D_{\mu}, D_{\nu}\right]$ is always vanishing when applied to $S U(2)$ invariants (right-handed fermions, $B$ and $G$ fields, $\mathcal{F}(h)$ functions), while it is traded for a field strength when it acts on a quantity $X$ with non-trivial isospin transformations: $\left[D_{\mu}, D_{\nu}\right] X=i g\left[W_{\mu \nu}, X\right]$.

- further combinations of $\mathbf{T}$ and $\mathbf{V}_{\mu}$ that do not appear in the basis reported in Sect. 2.3 have been traded for others using the identities (C.1) and (C.4).

\section{C.3 Construction of $\Delta \mathscr{L}_{4 F}$}

Further details of the construction and of the reduction of the four-fermion operators basis are provided in this section. None of the terms of $\Delta \mathscr{L}_{4 F}$ have been removed via the EOMs, while the Fierz identities (C.7)-(C.10) have been extensively employed for removing redundant structures. In particular, operators with tensor currents $\left(\left(\bar{\psi} \sigma^{\mu \nu} \psi\right)^{2}\right)$ were not included in the final basis, as they are always equivalent to combinations of scalar contractions via the Fierz identity (C.7). Similarly, operators with the scalar contraction $\left(\bar{\psi}_{L} \psi_{R}\right)\left(\bar{\psi}_{R} \psi_{L}\right)$ have been traded for terms with the vector structure $\left(\bar{\psi}_{L} \gamma_{\mu} \psi_{L}\right)\left(\bar{\psi}_{R} \gamma^{\mu} \psi_{R}\right)$ employing the identity (C.8).

\section{Four-quark (lepton) operators}

- There are four independent $S U$ (2) contractions of four quarks that can be constructed with the scalar structure $\left(\bar{\psi}_{L} \psi_{R}\right)\left(\bar{\psi}_{L} \psi_{R}\right)$. They are easily identified in unitary gauge by the $U(1)_{\mathrm{em}}$ invariants

$(u u)(u u), \quad(d d)(d d), \quad(u u)(d d), \quad(u d)(d u)$.

Keeping colour contractions into account, the total number of independent operators in this category is eight.

With four leptons there is only one invariant with this Lorentz structure, due to the absence of right-handed neutrinos: $(e e)(e e)$.

We do not provide the expressions of all the possible $S U$ (2) structures in terms of the invariants selected for the basis of Sect. 2.3. However, it is worth commenting on two contractions that can be constructed without the explicit insertion of Goldstone bosons: in the four-quark case they are

$$
\begin{aligned}
& R_{\varepsilon 1}^{\mathcal{Q}}=\varepsilon_{i j} \varepsilon_{a b}\left(\bar{Q}_{L i} Q_{R a}\right)\left(\bar{Q}_{L j} Q_{R b}\right) \mathcal{F} \\
& R_{\varepsilon 2}^{\mathcal{Q}}=\varepsilon_{i j} \varepsilon_{a b}\left(\bar{Q}_{L i} \lambda^{A} Q_{R a}\right)\left(\bar{Q}_{L j} \lambda^{A} Q_{R b}\right) \mathcal{F}
\end{aligned}
$$

In the four-lepton case, it is possible to introduce a structure analogous to the first one, but only in the presence of right-handed neutrinos. This would read

$R_{\varepsilon N}^{\ell}=\varepsilon_{i j} \varepsilon_{a b}\left(\bar{L}_{L i} L_{R a}\right)\left(\bar{L}_{L j} L_{R b}\right) \mathcal{F}$.

The operators of Eqs. (C.16) and (C.17) are redundant in the basis of Sect. 2.3: in fact, exploiting the properties of the Pauli matrices and the completeness relation (C.11) one has

$$
\mathbf{U}_{i a} \mathbf{U}_{j b}-\left(\mathbf{U} \sigma^{k}\right)_{i a}\left(\mathbf{U} \sigma^{k}\right)_{j b}=2 \varepsilon_{i j} \varepsilon_{a b}\left(c_{\pi}^{2}+\frac{|\vec{\pi}|^{2}}{v^{2}} s_{\pi}^{2}\right),
$$

where we have used the compact notation $s_{\pi} \equiv \sin$ $(|\vec{\pi}| / v), c_{\pi} \equiv \cos (|\vec{\pi}| / v)$. From Eq. (C.18) it follows immediately that

$$
\begin{aligned}
\frac{R_{1}^{\mathcal{Q}}-R_{2}^{\mathcal{Q}}}{2} & =R_{\varepsilon 1}^{\mathcal{Q}}\left(c_{\pi}^{2}+\frac{|\vec{\pi}|^{2}}{v^{2}} s_{\pi}^{2}\right) \\
& =R_{\varepsilon 1}^{\mathcal{Q}}\left(1-\frac{|\vec{\pi}|^{2}}{v^{2}}+\frac{4}{3} \frac{|\vec{\pi}|^{4}}{v^{4}}+\cdots\right) \\
\frac{R_{5}^{\mathcal{Q}}-R_{6}^{\mathcal{Q}}}{2} & =R_{\varepsilon 2}^{\mathcal{Q}}\left(c_{\pi}^{2}+\frac{|\vec{\pi}|^{2}}{v^{2}} s_{\pi}^{2}\right) \\
& =R_{\varepsilon 2}^{\mathcal{Q}}\left(1-\frac{|\vec{\pi}|^{2}}{v^{2}}+\frac{4}{3} \frac{|\vec{\pi}|^{4}}{v^{4}}+\cdots\right) \\
\frac{R_{1}^{\ell}-R_{8}^{\ell}}{2} & =R_{\varepsilon N}^{\ell}\left(c_{\pi}^{2}+\frac{|\vec{\pi}|^{2}}{v^{2}} s_{\pi}^{2}\right) \\
& =R_{\varepsilon N}^{\ell}\left(1-\frac{|\vec{\pi}|^{2}}{v^{2}}+\frac{4}{3} \frac{|\vec{\pi}|^{4}}{v^{4}}+\cdots\right) .
\end{aligned}
$$

Therefore, the interactions contained in $R_{\varepsilon 1}^{\mathcal{Q}}, R_{\varepsilon 2}^{\mathcal{Q}}$ and $R_{\varepsilon N}^{\ell}$ are already described by linear combinations of operators in the basis.

- The class of four-fermion operators with two left-handed currents contains four independent operators in both the four-quark and the four-lepton cases:

$$
\begin{aligned}
& \left(\bar{Q}_{L} \gamma_{\mu} Q_{L}\right)^{2}: \quad(u u)(u u),(d d)(d d), \\
& (u u)(d d),(u d)(d u), \\
& \left(\bar{L}_{L} \gamma_{\mu} L_{L}\right)^{2}: \quad(v v)(v v),(e e)(e e),(v v)(e e), \\
& (v e)(e v) .
\end{aligned}
$$

Notice that in this case the octet colour contraction $\left(\bar{Q}_{L} \gamma_{\mu} \lambda^{A} Q_{L}\right)^{2}$ is not independent. In fact it is equivalent to a combination of invariants with singlet colour contractions. Using Eqs. (C.9) and (C.12): 


$$
\begin{aligned}
& \left(\bar{Q}_{L} \gamma_{\mu} \lambda^{a} Q_{L}\right)\left(\bar{Q}_{L} \gamma_{\mu} \lambda^{a} Q_{L}\right) \\
& =\frac{1}{3}\left(\bar{Q}_{L} \gamma_{\mu} Q_{L}\right)\left(\bar{Q}_{L} \gamma_{\mu} Q_{L}\right) \\
& \quad+\left(\bar{Q}_{L} \gamma_{\mu} \sigma^{j} Q_{L}\right)\left(\bar{Q}_{L} \gamma_{\mu} \sigma^{j} Q_{L}\right) .
\end{aligned}
$$

An analogous relation holds for the structures with righthanded currents.

- The class of four-fermion operators with two righthanded currents contains four independent operators in the four-quark case but only one in the four-lepton sector:

$$
\begin{aligned}
& \left(\bar{Q}_{R} \gamma_{\mu} Q_{R}\right)^{2}: \quad(u u)(u u),(d d)(d d),(u u)(d d), \\
& \quad(u d)(d u), \\
& \left(\bar{L}_{R} \gamma_{\mu} L_{R}\right)^{2}: \quad(e e)(e e) .
\end{aligned}
$$

- Finally, there are five independent $S U(2)$ contractions for quark vector currents of opposite chirality $\left(\bar{\psi}_{L} \gamma_{\mu} \psi_{L}\right)$ $\left(\bar{\psi}_{R} \gamma^{\mu} \psi_{R}\right)$, to be doubled when including octet colour contractions:

$$
\begin{aligned}
& (u u)(u u), \quad(d d)(d d), \quad(u u)(d d), \quad(d d)(u u), \\
& (u d)(d u)+(d u)(u d) .
\end{aligned}
$$

The four-lepton counterpart, instead, contains two invariants corresponding to the interactions

$(e e)(e e), \quad(v v)(e e)$.

\section{Mixed quark-lepton operators}

- Operators with the scalar contraction $\left(\bar{\psi}_{L} \psi_{R}\right)\left(\bar{\psi}_{L} \psi_{R}\right)$ can have either the structure $(\bar{Q} Q)(\bar{L} L)$ or $(\bar{Q} L)(\bar{L} Q)$. Each of these yield three independent invariants, that are most easily identified in unitary gauge by the interactions:

$$
\begin{array}{llll}
(\bar{Q} Q)(\bar{L} L): & (u u)(e e), & (d d)(e e), & (d u)(v e), \\
(\bar{Q} L)(\bar{L} Q): & (u e)(e u), & (d e)(e d), & (d e)(v u) .
\end{array}
$$

- The two combinations $\left(\bar{Q}_{L} \gamma_{\mu} Q_{L}\right)\left(\bar{L}_{L} \gamma^{\mu} L_{L}\right)$, $\left(\bar{Q}_{L} \gamma_{\mu} L_{L}\right)\left(\bar{L}_{L} \gamma^{\mu} Q_{L}\right)$ are related by the Fierz identity (C.9), and therefore only the former structure has been retained. The same holds for the analogous terms constructed with right-handed currents, that are connected by Eq. (C.10).

This class includes five independent left-handed invariants, identified by the hermitian combinations

$$
(u u)(e e), \quad(d d)(e e), \quad(u u)(v v), \quad(d d)(v v),
$$$$
(d u)(v e)+(u d)(e v) \text {, }
$$

and two right-handed ones:

$(u u)(e e), \quad(d d)(e e)$.

- Operators with one left-handed and one right-handed current can be constructed in either of the combinations $\left(\bar{Q}_{L} \gamma_{\mu} Q_{L}\right)\left(\bar{L}_{R} \gamma^{\mu} L_{R}\right),\left(\bar{L}_{L} \gamma_{\mu} L_{L}\right)\left(\bar{Q}_{R} \gamma^{\mu} Q_{R}\right)$ and $\left(\bar{Q}_{L} \gamma_{\mu} L_{L}\right)\left(\bar{L}_{R} \gamma^{\mu} Q_{R}\right)$. These provide, respectively, 2 $+5+3$ independent interactions:

$$
\begin{array}{ll}
(\bar{Q} Q)(\bar{L} L): & (u u)(e e), \quad(d d)(e e), \\
(\bar{L} L)(\bar{Q} Q): & (e e)(u u), \quad(e e)(d d), \quad(v v)(u u), \\
& (v v)(d d),(v e)(d u)+(e v)(u d), \\
(\bar{L} Q)(\bar{Q} L): & (e u)(u e), \quad(e d)(d e), \quad(v u)(d e) .
\end{array}
$$

\section{Application of the EOMs}

Given the LO Lagrangian in Eq. (2.3), the fields satisfy the following EOMs:

$$
\begin{gathered}
i \not D \psi_{L}=\frac{v}{\sqrt{2}} \mathbf{U} \mathcal{Y}_{\psi}(h) \psi_{R}, \\
i \not D \psi_{R}=\frac{v}{\sqrt{2}} \mathcal{Y}_{Q}^{\dagger}(h) \mathbf{U}^{\dagger} \psi_{L}, \\
\left(D^{\mu} W_{\mu \nu}\right)^{a}=\sum_{\psi=Q, L} \frac{g}{2} \bar{\psi}_{L} \sigma^{a} \gamma_{\nu} \psi_{L} \\
+\frac{i g v^{2}}{4} \operatorname{Tr}\left[\mathbf{V}_{\nu} \sigma^{a}\right] \mathcal{F}_{C}(h), \\
\partial^{\mu} B_{\mu \nu}=g c_{\theta} \sum_{i=L, R} \bar{\psi}_{i} \mathbf{h}_{\psi_{i}} \gamma_{\nu} \psi_{i} \\
-\frac{i g c_{\theta} v^{2}}{4} \operatorname{Tr}\left[\mathbf{T} \mathbf{V}_{\mu}\right] \mathcal{F}_{C}(h), \\
\square h=-V^{\prime}(h)-\frac{v^{2}}{4} \operatorname{Tr}\left[\mathbf{V}_{\mu} \mathbf{V}^{\mu}\right] \mathcal{F}_{C}^{\prime}(h) \\
-\sum_{\psi=Q, L} \frac{v}{\sqrt{2}}\left(\bar{\psi}_{L} \mathbf{U} \mathcal{Y}_{\psi}^{\prime}(h) \psi_{R}+\text { h.c. }\right),
\end{gathered}
$$

where $\mathbf{h}_{\psi_{i}}$ are the hypercharges in the $2 \times 2$ matrix notation:

$h_{Q_{L}}=\operatorname{diag}(1 / 6,1 / 6), \quad h_{Q_{R}}=\operatorname{diag}(2 / 3,-1 / 3)$,

$h_{L_{L}}=\operatorname{diag}(-1 / 2,-1 / 2), \quad h_{L_{R}}=\operatorname{diag}(0,-1)$,

and the prime denotes the first derivative with respect to $h$. A consequence of Eqs. (D.2) and (D.1) is 


$$
\begin{aligned}
& \mathbf{D}_{\mu}\left(\mathbf{V}^{\mu} \mathcal{F}_{C}\right)=\frac{i}{v^{2}} D_{\mu}\left(\sum_{\psi=Q, L} \bar{\psi}_{L} \sigma^{j} \gamma^{\mu} \psi_{L}\right) \sigma^{j} \\
& =\frac{1}{\sqrt{2} v} \sum_{\psi=Q, L}\left(\bar{\psi}_{L} \sigma^{j} \mathbf{U} \mathcal{Y}_{\psi}(h) \psi_{R}-\bar{\psi}_{R} \mathcal{Y}_{\psi}^{\dagger}(h) \mathbf{U}^{\dagger} \sigma^{j} \psi_{L}\right) \sigma^{j},
\end{aligned}
$$

which can be recast in the form

$$
\begin{aligned}
& \operatorname{Tr}\left(\sigma^{j} \mathbf{D}_{\mu} \mathbf{V}^{\mu}\right) \mathcal{F}(h)=\frac{\sqrt{2}}{v} \\
& \quad \times \sum_{\psi=Q, L}\left(\bar{\psi}_{L} \sigma^{j} \mathbf{U} \mathcal{Y}_{\psi}(h) \psi_{R}-\bar{\psi}_{R} \mathcal{Y}_{\psi}^{\dagger}(h) \mathbf{U}^{\dagger} \sigma^{j} \psi_{L}\right) \\
& \quad-\operatorname{Tr}\left(\sigma^{j} \mathbf{V}_{\mu}\right) \partial^{\mu} \mathcal{F}(h),
\end{aligned}
$$

which is valid order by order in the $h$ expansion.

\section{D.1 Operators that have been removed via EOM}

The EOMs relate the purely bosonic and the fermionic sectors, and they have been used to eliminate operators that are redundant when both sectors are considered at the same time. In this section we list the categories of operators that have been removed.

\section{Bosonic sector}

- Operators containing $\square \mathcal{F}(h)$. Applying the EOM for the Higgs, Eq. (D.4), these terms can be traded for a combination of other bosonic operators plus fermionic bilinears and four-fermion operators. The following $\mathrm{CP}$ even terms have been removed, compared to the basis of Ref. [24]:

$$
\begin{aligned}
& \mathcal{P}_{\square H}(h)=\frac{\square h \square h}{v^{2}} \mathcal{F}, \\
& \mathcal{P}_{7}(h)=\operatorname{Tr}\left(\mathbf{V}_{\mu} \mathbf{V}^{\mu}\right) \square \mathcal{F}, \\
& \mathcal{P}_{25}(h)=\operatorname{Tr}\left(\mathbf{T V}_{\mu}\right) \operatorname{Tr}\left(\mathbf{T} \mathbf{V}^{\mu}\right) \square \mathcal{F},
\end{aligned}
$$

and the CP odd operator

$$
\mathcal{S}_{13}=\operatorname{Tr}\left(\mathbf{T V}_{\mu}\right) \partial^{\mu} \square \mathcal{F}
$$

- Operators containing $\mathbf{D}_{\mu} \mathbf{V}^{\mu}$.

Rewriting the traceless matrix $\mathbf{D}_{\mu} \mathbf{V}^{\mu}$ as

$$
\mathbf{D}_{\mu} \mathbf{V}^{\mu}=\frac{\sigma^{a}}{2} \operatorname{Tr}\left(\sigma^{a} \mathbf{D}_{\mu} \mathbf{V}^{\mu}\right)
$$

and applying the identity (D.7), these bosonic operators can be traded by combinations of fermion bilinears, fourfermion operators and other bosonic terms that already belong to the basis. The following $\mathrm{CP}$ even terms have been eliminated, in the notation of Ref. [24]:

$$
\begin{aligned}
\mathcal{P}_{9}(h) & =\operatorname{Tr}\left(\left(\mathbf{D}_{\mu} \mathbf{V}^{\mu}\right)^{2}\right) \mathcal{F}, \\
\mathcal{P}_{10}(h) & =\operatorname{Tr}\left(\mathbf{V}_{\nu} \mathbf{D}_{\mu} \mathbf{V}^{\mu}\right) \partial^{\nu} \mathcal{F}, \\
\mathcal{P}_{15}(h) & =\operatorname{Tr}\left(\mathbf{T} \mathbf{D}_{\mu} \mathbf{V}^{\mu}\right) \operatorname{Tr}\left(\mathbf{T} \mathbf{D}_{v} \mathbf{V}^{\nu}\right) \mathcal{F}, \\
\mathcal{P}_{16}(h) & =\operatorname{Tr}\left(\left[\mathbf{T}, \mathbf{V}_{\nu}\right] \mathbf{D}_{\mu} \mathbf{V}^{\mu}\right) \operatorname{Tr}\left(\mathbf{T} \mathbf{V}^{\nu}\right) \mathcal{F}, \\
\mathcal{P}_{19}(h) & =\operatorname{Tr}\left(\mathbf{T} \mathbf{D}_{\mu} \mathbf{V}^{\mu}\right) \operatorname{Tr}\left(\mathbf{T} \mathbf{V}_{\nu}\right) \partial^{\nu} \mathcal{F} .
\end{aligned}
$$

Analogously, five CP odd operators have been traded for others: in the notation of Ref. [25] they are

$$
\begin{aligned}
& \mathcal{S}_{10}=i \operatorname{Tr}\left(\mathbf{V}_{\nu} \mathbf{D}_{\mu} \mathbf{V}^{\mu}\right) \operatorname{Tr}\left(\mathbf{T} \mathbf{V}^{v}\right) \mathcal{F}, \\
& \mathcal{S}_{11}=i \operatorname{Tr}\left(\mathbf{T D}_{\mu} \mathbf{V}^{\mu}\right) \operatorname{Tr}\left(\mathbf{V}_{\nu} \mathbf{V}^{v}\right) \mathcal{F}, \\
& \mathcal{S}_{12}=i \operatorname{Tr}\left(\left[\mathbf{V}^{\mu}, \mathbf{T}\right] \mathbf{D}_{\nu} \mathbf{V}^{v}\right) \partial_{\mu} \mathcal{F}, \\
& \mathcal{S}_{14}=i \operatorname{Tr}\left(\mathbf{T D}_{\mu} \mathbf{V}^{\mu}\right) \partial_{\nu} \mathcal{F}(h) \partial^{\nu} \mathcal{F}^{\prime}, \\
& \mathcal{S}_{16}=i \operatorname{Tr}\left(\mathbf{T D}_{\mu} \mathbf{V}^{\mu}\right) \operatorname{Tr}\left(\mathbf{T} \mathbf{V}_{\nu}\right) \operatorname{Tr}\left(\mathbf{T} \mathbf{V}^{v}\right) \mathcal{F} .
\end{aligned}
$$

\section{Fermionic sector}

- Bilinears of the type $\bar{\psi} \Gamma \gamma_{\mu} \psi \partial^{\mu} \mathcal{F}$. Applying the EOMs for fermions (Eq. (D.1)), these operators can be schematically rewritten as

$$
\begin{aligned}
\bar{\psi} \Gamma \gamma_{\mu} \psi \partial^{\mu} \mathcal{F}= & -\bar{\psi} \overleftarrow{D} \Gamma \psi \mathcal{F}-\bar{\psi} \gamma_{\mu}\left(D^{\mu} \Gamma\right) \psi \mathcal{F} \\
& -\bar{\psi} \Gamma \not D \psi \mathcal{F} \\
\rightarrow & \bar{\psi} \gamma_{\mu}\left(D^{\mu} \Gamma\right) \psi \mathcal{F} \bar{\psi} \Gamma \psi \mathcal{F}
\end{aligned}
$$

- Bilinears containing $\square \mathcal{F}$.

Operators in this category are removed applying the EOM for the Higgs field, Eq. (D.4) and traded for other bilinears plus four-fermion operators.

- invariants containing $\mathbf{D}_{\mu} \mathbf{V}^{\mu}$

As in the bosonic sector, these operators are removed applying the identity (D.7). and traded for other bilinears plus four-fermion operators.

- Finally, the EOMs for the gauge (Eqs. (D.2), (D.3)) and Higgs (Eq. (D.4)) fields imply the following additional relations (signs and numeric coefficients not specified):

$$
\begin{aligned}
& \mathcal{P}_{B}+\mathcal{P}_{1}+\mathcal{P}_{2}+\mathcal{P}_{4}+\mathcal{P}_{T} \\
& \rightarrow i \bar{L}_{L_{i}} \gamma_{\mu}\left\{\mathbf{V}^{\mu}, \mathbf{T}\right\} L_{L_{i}} \mathcal{F}+\mathcal{N}_{5}^{\mathcal{Q}}+\mathcal{N}_{6}^{\mathcal{Q}}, \\
& \mathcal{P}_{W}+\mathcal{P}_{1}+\mathcal{P}_{3}+\mathcal{P}_{5}+\operatorname{Tr}\left(\mathbf{V}_{\mu} \mathbf{V}^{\mu}\right) \mathcal{F} \\
& \rightarrow i \bar{L}_{L_{i}} \gamma_{\mu} \mathbf{V}^{\mu} L_{L_{i}} \mathcal{F}+\mathcal{N}_{1}^{\mathcal{Q}}, \\
& \mathcal{P}_{T}+\mathcal{P}_{1}+\mathcal{P}_{3}+\mathcal{P}_{12}+\mathcal{P}_{13}+\mathcal{P}_{17} \\
& \rightarrow i \bar{L}_{L_{i}} \gamma_{\mu} \mathbf{T} \mathbf{V}^{\mu} \mathbf{T} L_{L_{i}} \mathcal{F}+i \bar{L}_{L_{i}} \gamma_{\mu} \mathbf{V}^{\mu} L_{L_{i}} \mathcal{F}+\mathcal{N}_{7}^{\mathcal{Q}} \\
& \quad+\mathcal{N}_{1}^{\mathcal{Q}} .
\end{aligned}
$$


These have been employed to remove the three (flavourdiagonal contractions of the) leptonic operators specified on the right-hand side. This choice simplifies the renormalisation procedure.

\section{E Feynman rules}

This appendix provides a complete list of all the Feynman rules resulting from both fermionic and bosonic operators considered in the present work and listed in Sects. 2.2 and 2.3. For compactness we omit CP violating terms, that are not relevant for the phenomenological study presented. The rules are derived in unitary gauge and only vertices with up to four legs are shown. The SM contribution and the renormalisation effects are also included, up to first order in the effective coefficients. The latter are sometimes encoded in the quantities $\Delta g_{1}, \Delta g_{2}, \Delta g_{W}$ and $\Delta M_{W}$ defined in Eqs. (3.5), (3.6) in the text.

A few comments about the notation and conventions used:

- All momenta are flowing inwards and the convention $\partial_{\mu} \rightarrow-i p_{\mu}$ has been used in the derivation.
- We use a shorthand notation for the products $c_{i} a_{i}$ : for the bosonic operators, we replace $a_{i} c_{i} \rightarrow a_{i}$ and $b_{i} c_{i} \rightarrow a_{i}$. For the fermionic operators, we write $a_{i}^{f} n_{i}^{f} \rightarrow(n a)_{i}^{f}$. The structure $\bar{\psi} \psi \partial \mathcal{F} \partial \mathcal{F}^{\prime}$ gives couplings $h h f f$ with the coefficients $n_{i}^{f} a_{i}^{f} a_{i}^{\prime f}$. This notation has been shortened in $\left(n a a^{\prime}\right)_{i}^{f}$. For the coefficients of the function $\mathcal{F}_{C}(h)$, defined in Eq. (2.4), the notation $a_{C}=1+\Delta a_{C}, b_{C}=$ $1+\Delta b_{C}$ is adopted.

- We have fixed $V_{\mathrm{CKM}}=1$ for compactness. At the same level, all the effective coefficients are implicitly taken to be flavour diagonal.

- In the vertices with a single fermion current the spin contractions are obvious. For those with four fermions we use a notation with square brackets and lowercase indices: for example $\left[P_{R}\right]_{a b}\left[P_{L}\right]_{c d}$ means that the right chirality projector contracts the spins of the $a$ and $b$ particle, and the left chirality one shall be inserted between the $c$ and $d$ fields.

- Uppercase indices indicate colour and are assumed to be summed over when repeated. Whenever they are not specified, the colour (and flavour) contractions go with those of the spin. 


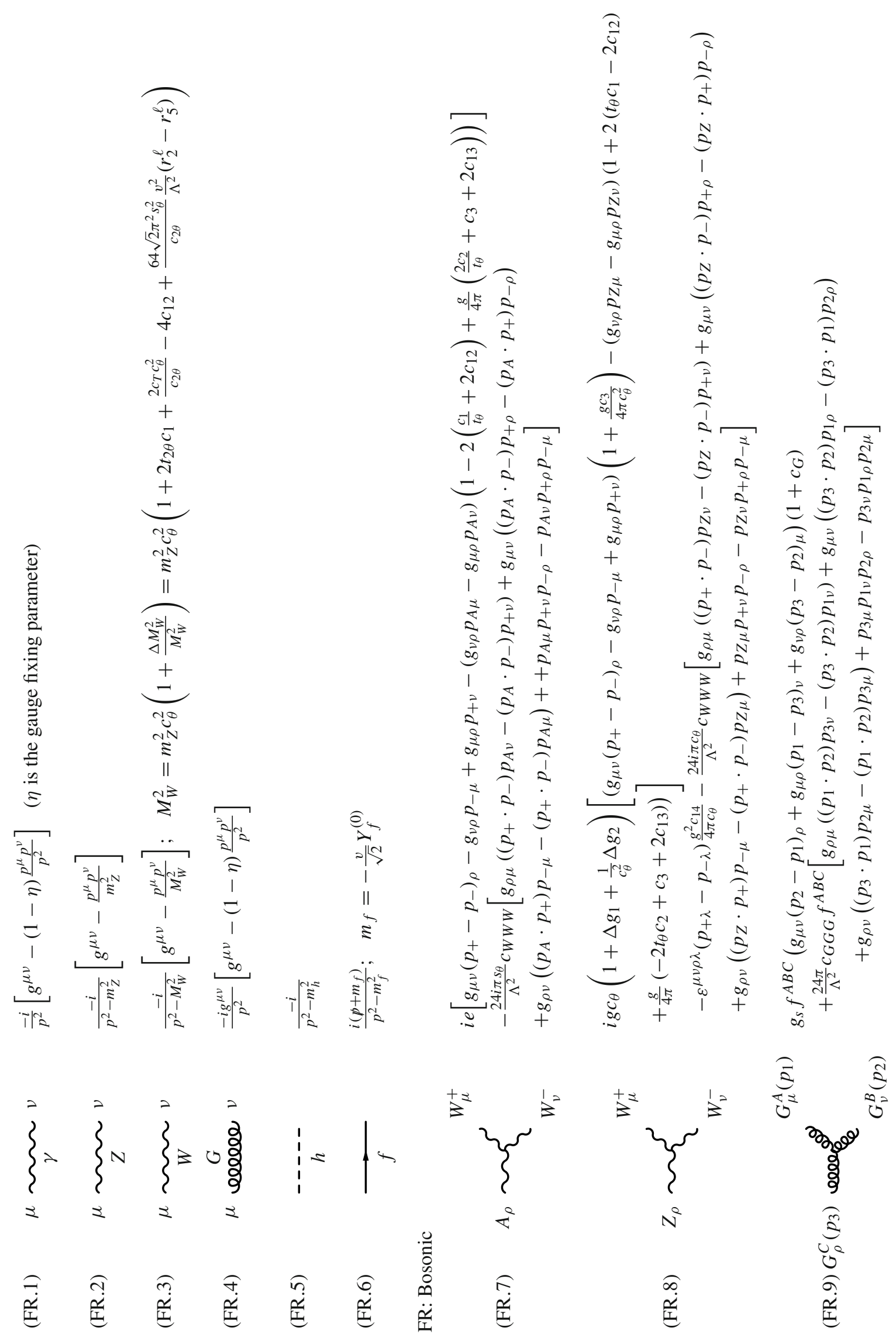




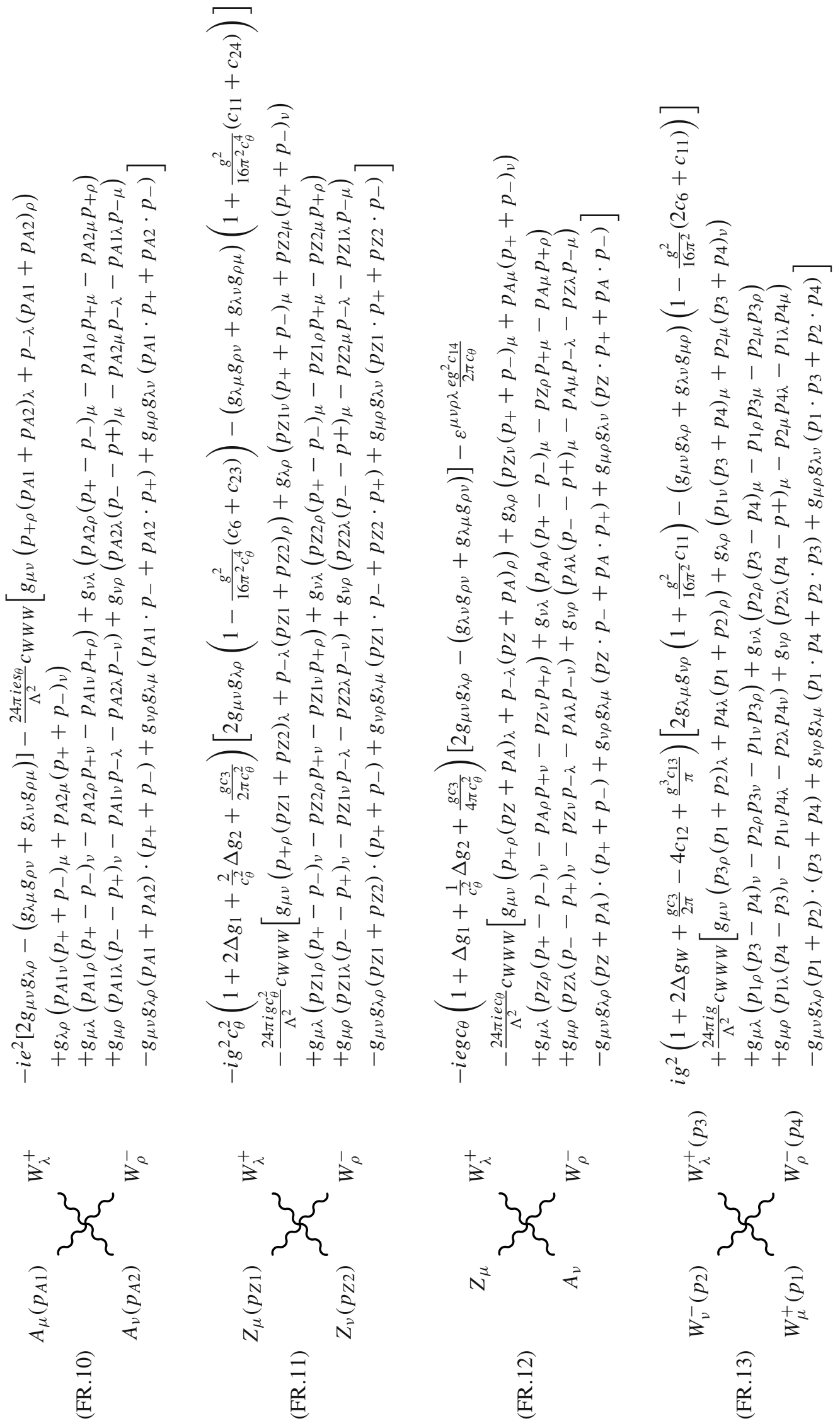



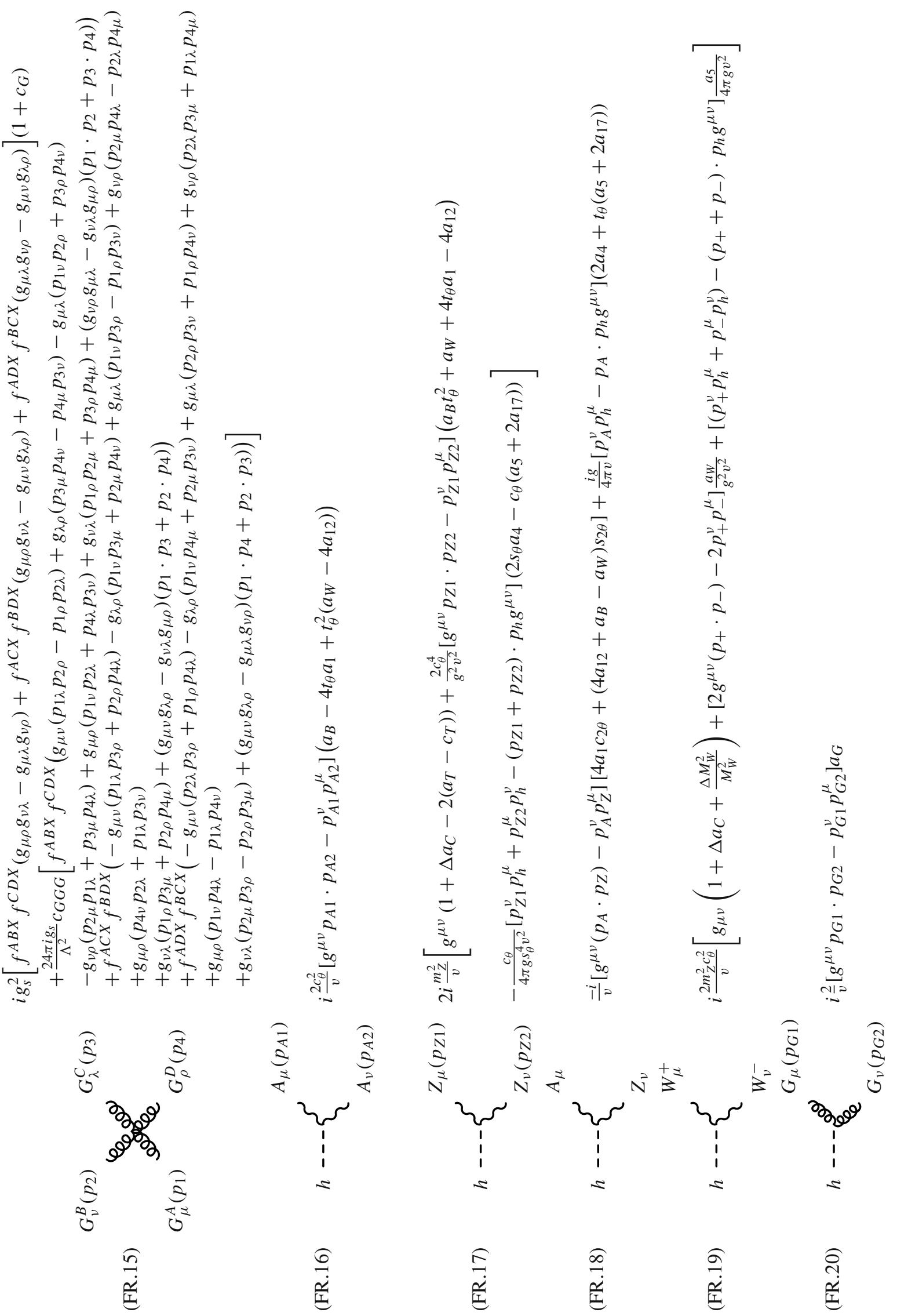


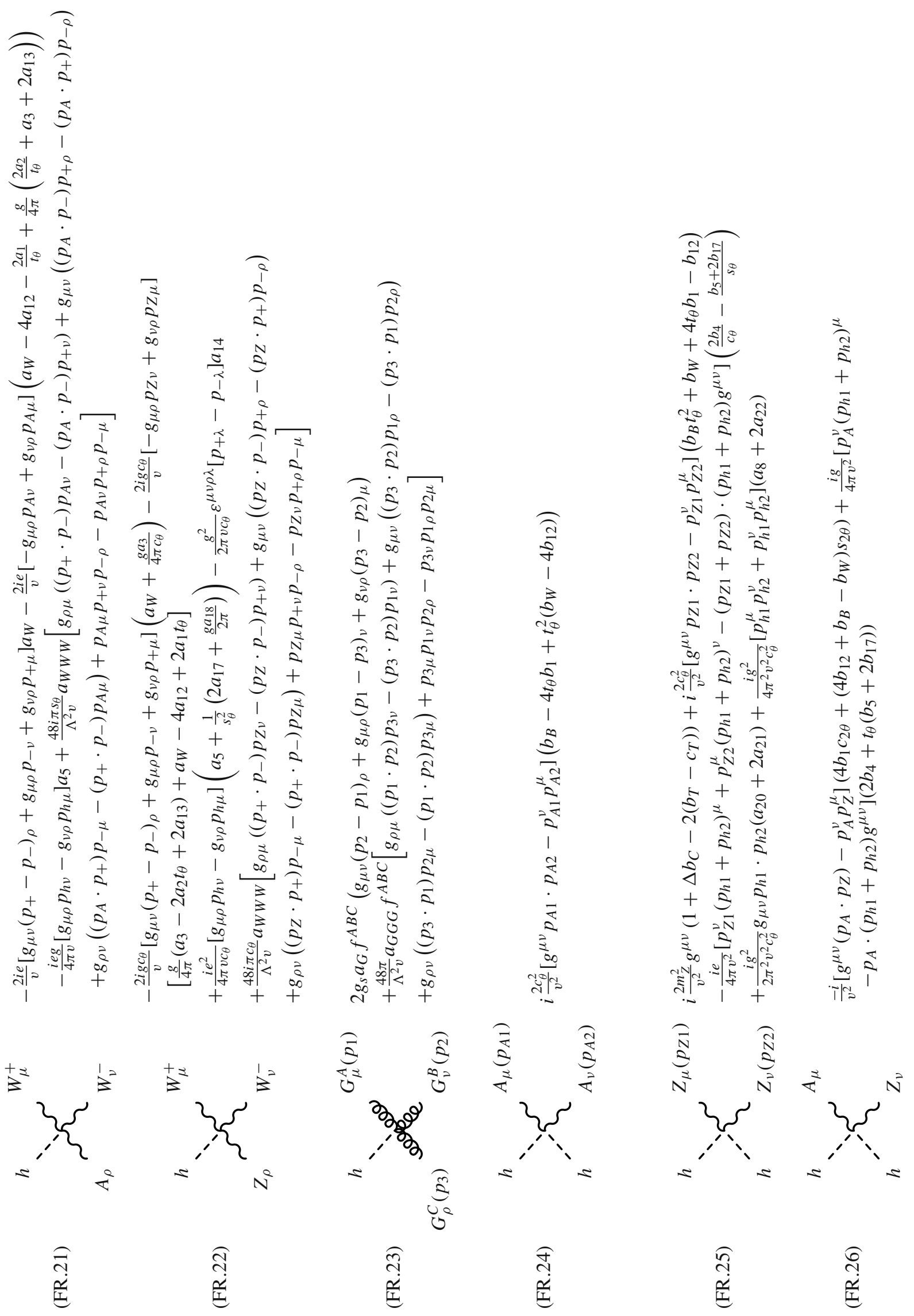




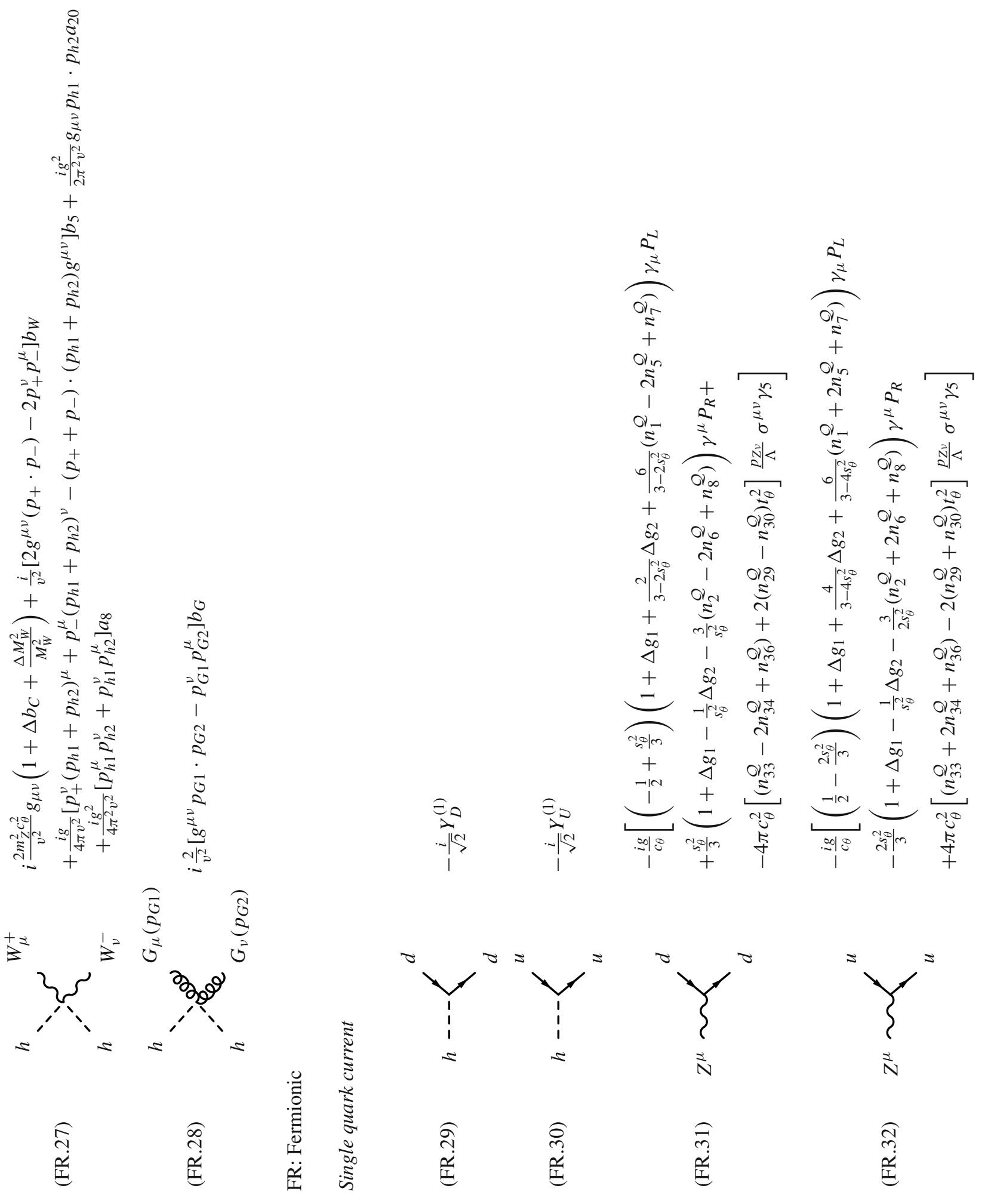



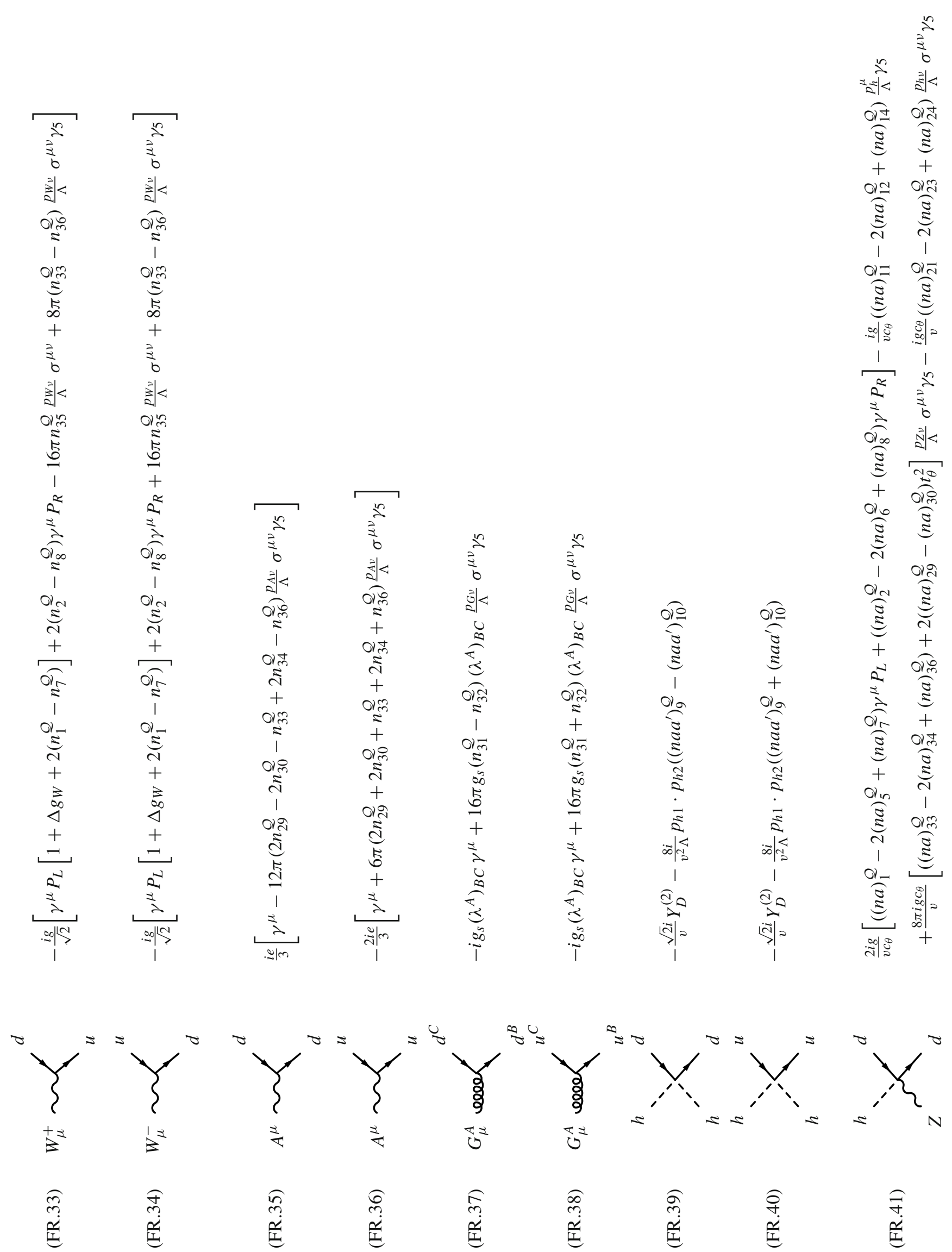

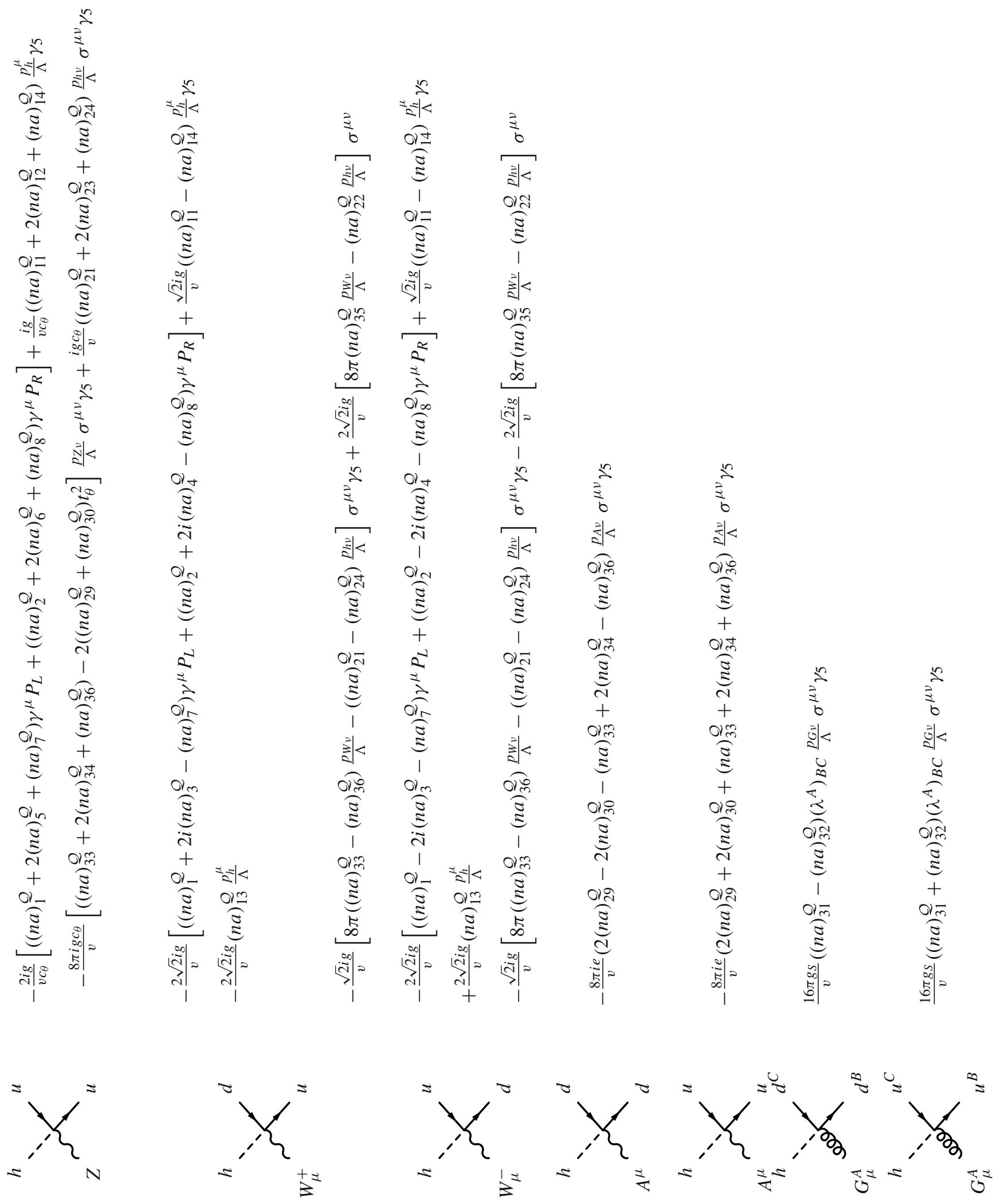

ชิ
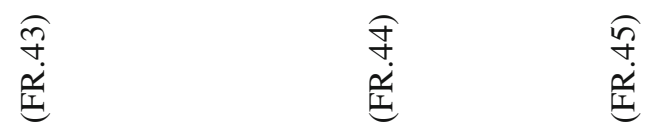

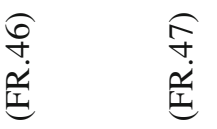

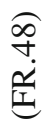




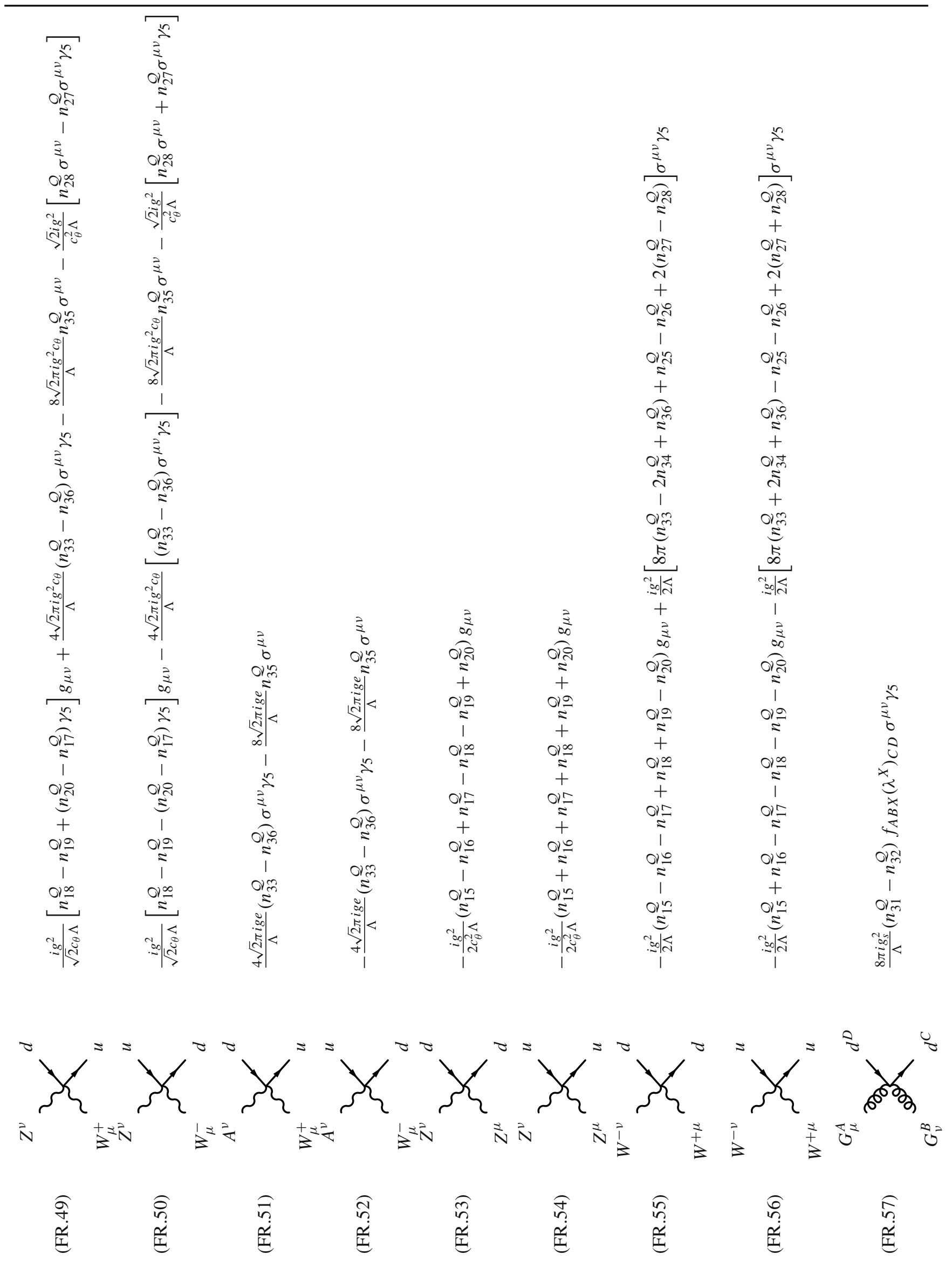



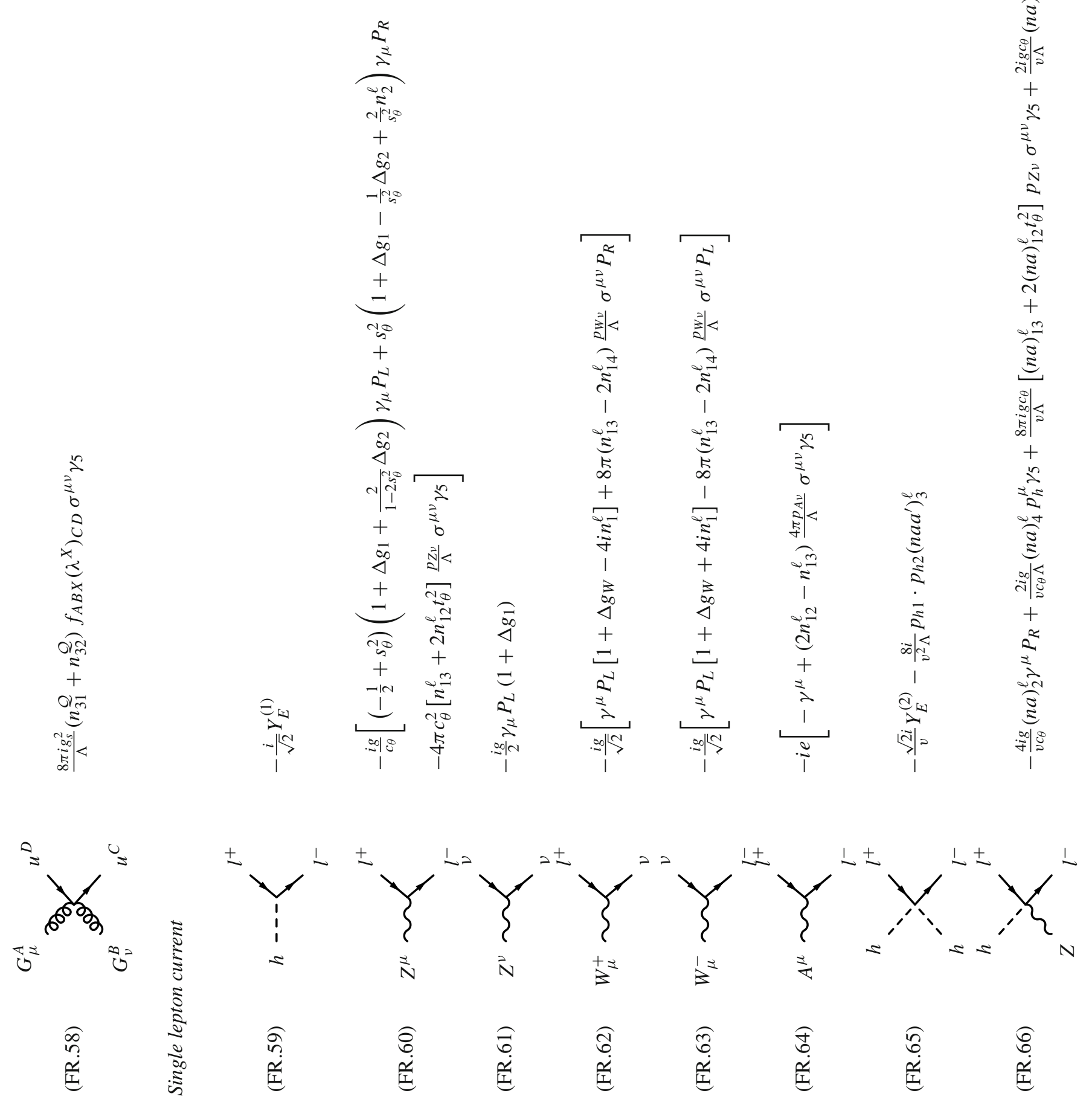

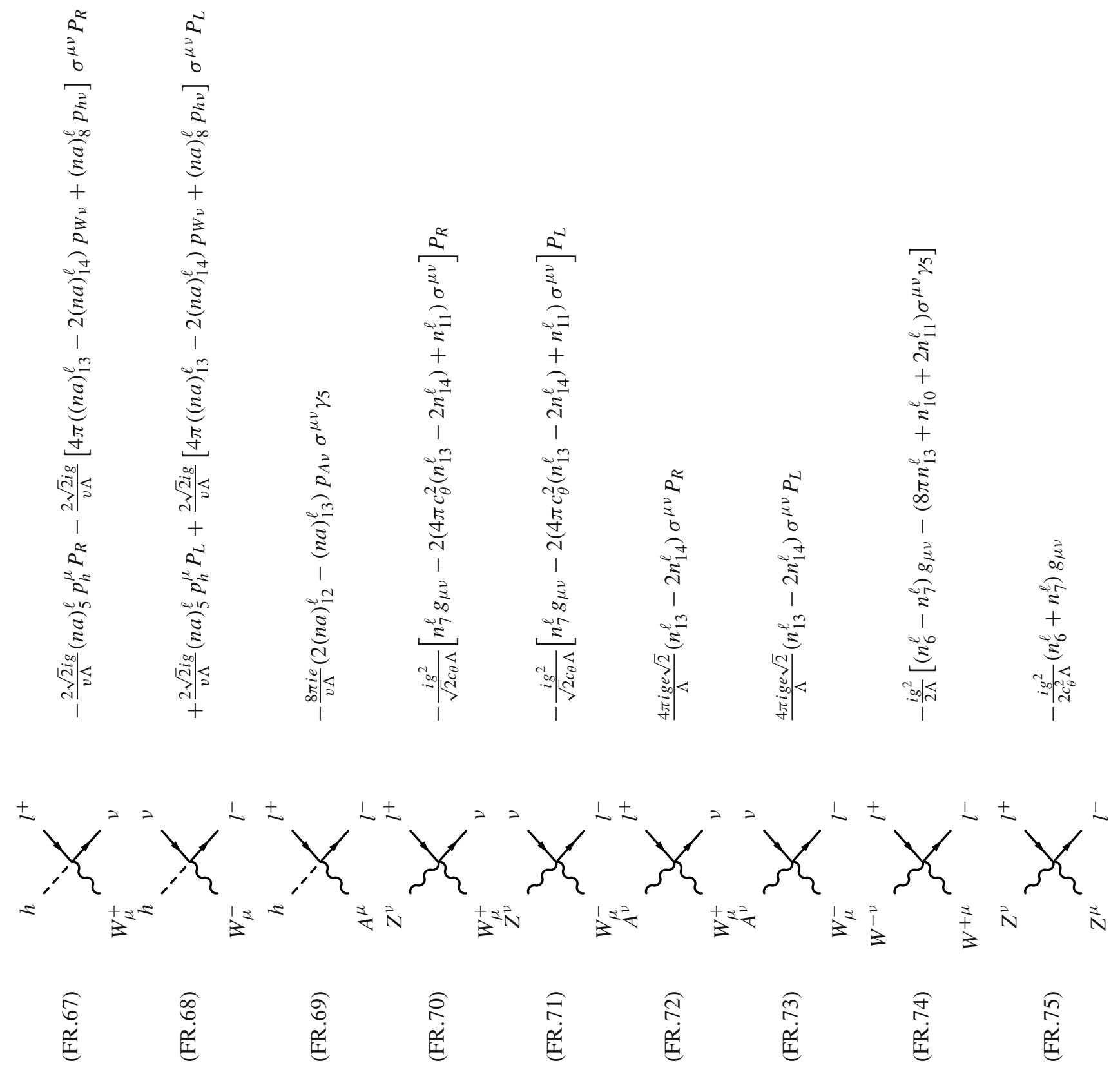


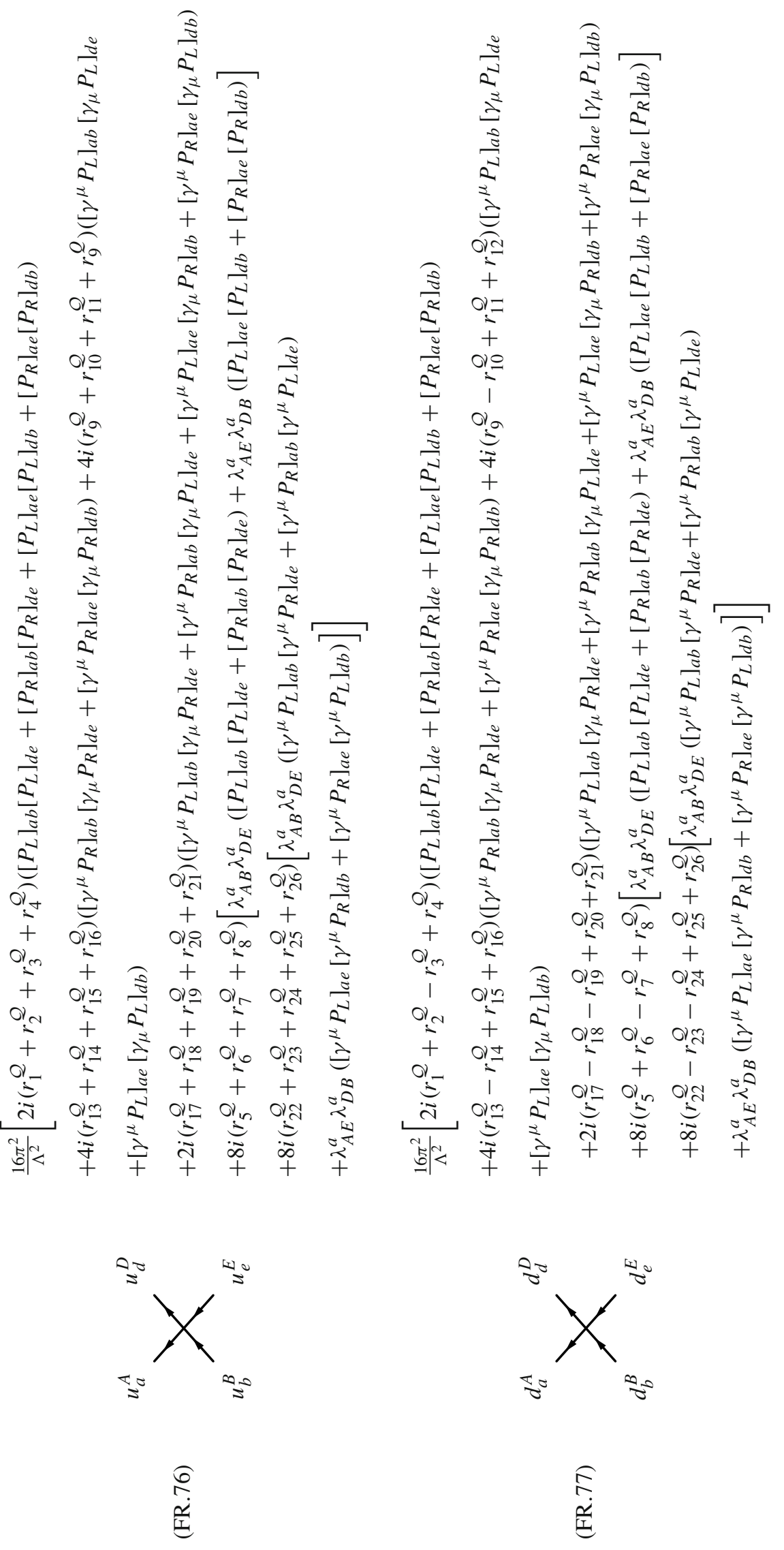



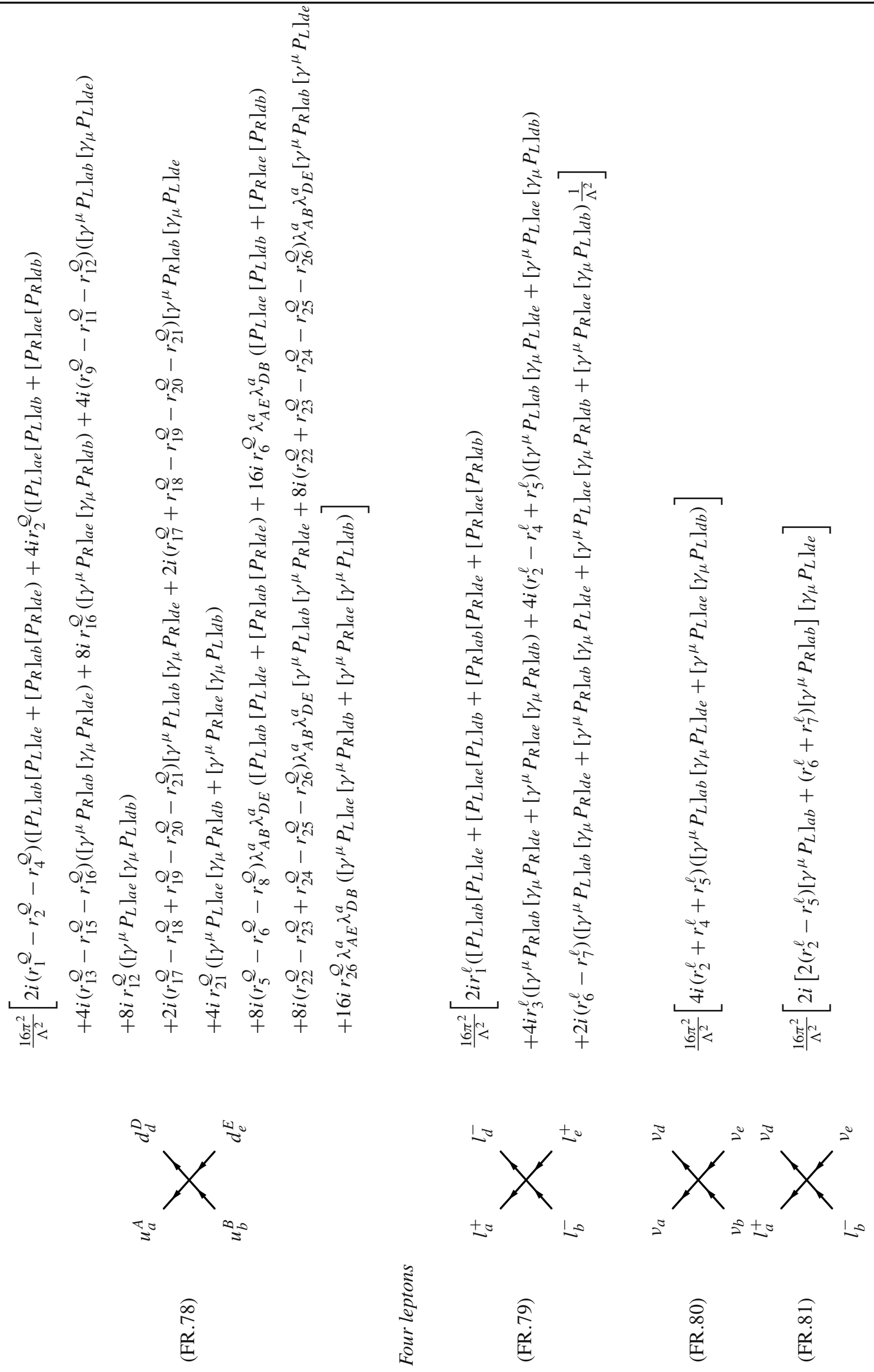


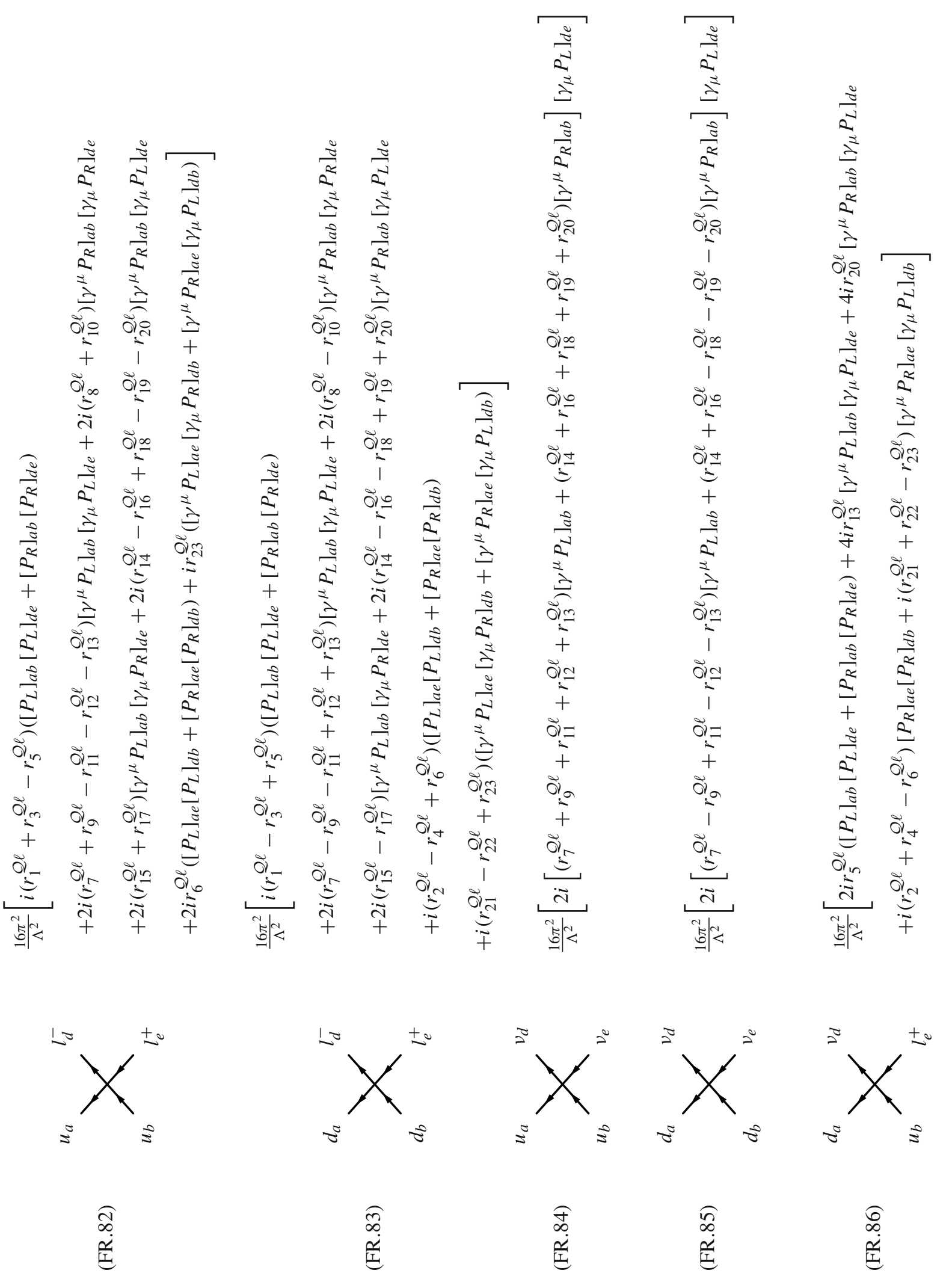




\section{References}

1. ATLAS Collaboration, G. Aad et al., Observation of a new particle in the search for the standard model Higgs boson with the ATLAS detector at the LHC. Phys. Lett. B 716, 1-29 (2012). arXiv: 1207.7214

2. CMS Collaboration, S. Chatrchyan et al., Observation of a new boson at a mass of $125 \mathrm{GeV}$ with the CMS experiment at the LHC. Phys. Lett. B 716, 30-61 (2012). arXiv:1207.7235

3. F. Englert, R. Brout, Broken symmetry and the mass of gauge vector mesons. Phys. Rev. Lett. 13, 321-323 (1964)

4. P.W. Higgs, Broken symmetries, massless particles and gauge fields. Phys. Lett. 12, 132-133 (1964)

5. P.W. Higgs, Broken symmetries and the masses of gauge bosons. Phys. Rev. Lett. 13, 508-509 (1964)

6. W. Buchmuller, D. Wyler, Effective Lagrangian analysis of new interactions and flavor conservation. Nucl. Phys. B 268, 621-653 (1986)

7. B. Grzadkowski, M. Iskrzynski, M. Misiak, J. Rosiek, Dimensionsix terms in the standard model Lagrangian. JHEP 10, 085 (2010). arXiv: 1008.4884

8. D.B. Kaplan, H. Georgi, $S U(2) \times U(1)$ breaking by vacuum misalignment. Phys. Lett. B 136, 183 (1984)

9. D.B. Kaplan, H. Georgi, S. Dimopoulos, Composite Higgs scalars. Phys. Lett. B 136, 187 (1984)

10. T. Banks, Constraints on $S U(2) \times U(1)$ breaking by vacuum misalignment. Nucl. Phys. B 243, 125 (1984)

11. K. Agashe, R. Contino, A. Pomarol, The minimal composite Higgs model. Nucl. Phys. B 719, 165-187 (2005). arXiv:hep-ph/0412089

12. B. Gripaios, A. Pomarol, F. Riva, J. Serra, Beyond the minimal composite Higgs model. JHEP 04, 070 (2009). arXiv:0902.1483

13. E. Halyo, Technidilaton or Higgs? Mod. Phys. Lett. A 8, 275-284 (1993)

14. W.D. Goldberger, B. Grinstein, W. Skiba, Distinguishing the Higgs boson from the dilaton at the large hadron collider. Phys. Rev. Lett. 100, 111802 (2008). arXiv:0708.1463

15. T. Appelquist, C.W. Bernard, Strongly interacting Higgs bosons. Phys. Rev. D 22, 200 (1980)

16. A.C. Longhitano, Heavy Higgs bosons in the Weinberg-Salam model. Phys. Rev. D 22, 1166 (1980)

17. A.C. Longhitano, Low-energy impact of a heavy Higgs boson sector. Nucl. Phys. B 188, 118 (1981)

18. F. Feruglio, The chiral approach to the electroweak interactions. Int. J. Mod. Phys. A 8, 4937-4972 (1993). arXiv:hep-ph/9301281

19. S. Weinberg, Phenomenological Lagrangians. Physica A 96, 327 (1979)

20. B. Grinstein, M. Trott, A Higgs-Higgs bound state due to new physics at a TeV. Phys. Rev. D 76, 073002 (2007). arXiv:0704.1505

21. R. Contino, C. Grojean, M. Moretti, F. Piccinini, R. Rattazzi, Strong double Higgs production at the LHC. JHEP 05, 089 (2010). arXiv: 1002.1011

22. R. Alonso, M.B. Gavela, L. Merlo, S. Rigolin, J. Yepes, The effective chiral Lagrangian for a light dynamical "Higgs particle". Phys. Lett. B 722 (2013) 330-335. arXiv:1212.3305 [Erratum: Phys. Lett. B 726, 926 (2013)]

23. R. Alonso, M.B. Gavela, L. Merlo, S. Rigolin, J. Yepes, Flavor with a light dynamical "Higgs particle". Phys. Rev. D 87(5), 055019 (2013). arXiv: 1212.3307

24. I. Brivio, T. Corbett, O. Éboli, M. Gavela, J. González-Fraile et al., Disentangling a dynamical Higgs. JHEP 1403, 024 (2014). arXiv: 1311.1823

25. M.B. Gavela, J. González-Fraile, M.C. González-García, L. Merlo, S. Rigolin, J. Yepes, CP violation with a dynamical Higgs. JHEP 10, 44 (2014). arXiv:1406.6367
26. G. Buchalla, O. Catà, C. Krause, Complete electroweak chiral Lagrangian with a light Higgs at NLO. Nucl. Phys. B 880, 552-573 (2014). arXiv:1307.5017

27. J. Yepes, Spin-1 resonances in a non-linear left-right dynamical Higgs context. arXiv:1507.03974

28. J. Yepes, R. Kunming, J. Shu, CP violation from spin-1 resonances in a left-right dynamical Higgs context. Commun. Theor. Phys. 66, 93-103 (2016). arXiv:1507.04745

29. F. Feruglio, B. Gavela, K. Kanshin, P.A.N. Machado, S. Rigolin, S. Saa, The minimal linear sigma model for the Goldstone Higgs. JHEP 06, 038 (2016). arXiv:1603.05668

30. R. Alonso, I. Brivio, B. Gavela, L. Merlo, S. Rigolin, Sigma decomposition. JHEP 12, 034 (2014). arXiv: 1409.1589

31. I.M. Hierro, L. Merlo, S. Rigolin, Sigma decomposition: the CPodd Lagrangian. JHEP 04, 016 (2016). arXiv:1510.07899

32. I. Brivio, O.J.P. Éboli, M.B. Gavela, M.C. González-Garcia, L. Merlo, S. Rigolin, Higgs ultraviolet softening. JHEP 12, 004 (2014). arXiv: 1405.5412

33. I. Brivio, M.B. Gavela, L. Merlo, K. Mimasu, J.M. No, R. del Rey, V. Sanz, Non-linear Higgs portal to dark matter. JHEP 04, 141 (2016). arXiv: 1511.01099

34. H. Murayama, V. Rentala, J. Shu, Probing strong electroweak symmetry breaking dynamics through quantum interferometry at the LHC. Phys. Rev. D 92(11), 116002 (2015). arXiv:1401.3761

35. R.L. Delgado, A. Dobado, F.J. Llanes-Estrada, One-loop $W_{L} W_{L}$ and $Z_{L} Z_{L}$ scattering from the electroweak Chiral Lagrangian with a light Higgs-like scalar. JHEP 02, 121 (2014). arXiv:1311.5993

36. R.L. Delgado, A. Dobado, M.J. Herrero, J.J. Sanz-Cillero, Oneloop $\gamma \gamma \rightarrow \mathrm{W}_{L}^{+} \mathrm{W}_{L}^{-}$and $\gamma \gamma \rightarrow \mathrm{Z}_{L} \mathrm{Z}_{L}$ from the electroweak Chiral Lagrangian with a light Higgs-like scalar. JHEP 07, 149 (2014). arXiv:1404.2866

37. R. Alonso, M.B. Gavela, L. Merlo, S. Rigolin, J. Yepes, Minimal flavour violation with strong Higgs dynamics. JHEP 06, 076 (2012). arXiv:1201.1511

38. B.M. Gavela, E.E. Jenkins, A.V. Manohar, L. Merlo, Analysis of general power counting rules in effective field theory. arXiv: 1601.07551

39. A. Manohar, H. Georgi, Chiral quarks and the nonrelativistic quark model. Nucl. Phys. B 234, 189 (1984)

40. A.G. Cohen, D.B. Kaplan, A.E. Nelson, Counting 4 Pis in strongly coupled supersymmetry. Phys. Lett. B 412, 301-308 (1997). arXiv:hep-ph/9706275

41. A. Biektter, A. Knochel, M. Krmer, D. Liu, F. Riva, Vices and virtues of Higgs effective field theories at large energy. Phys. Rev. D 91, 055029 (2015). arXiv: 1406.7320

42. R. Contino, A. Falkowski, F. Goertz, C. Grojean, F. Riva, On the validity of the effective field theory approach to SM precision tests. arXiv:1604.06444

43. H. Georgi, D.B. Kaplan, L. Randall, Manifesting the invisible axion at low-energies. Phys. Lett. B 169, 73 (1986)

44. L. Merlo, S. Saa, M. Sacristan, B and L non-conserving effective Lagrangian for a dynamical Higgs (to appear)

45. K. Hagiwara, S. Ishihara, R. Szalapski, D. Zeppenfeld, Low-energy effects of new interactions in the electroweak boson sector. Phys. Rev. D 48, 2182-2203 (1993)

46. K. Hagiwara, T. Hatsukano, S. Ishihara, R. Szalapski, Probing nonstandard bosonic interactions via $\mathrm{W}$ boson pair production at lepton colliders. Nucl. Phys. B 496, 66-102 (1997). arXiv:hep-ph/9612268

47. T. Corbett, O.J.P. Éboli, J. González-Fraile, M.C. González-Garcia, Constraining anomalous Higgs interactions. Phys. Rev. D 86, 075013 (2012). arXiv:1207.1344

48. T. Corbett, O.J.P. Éboli, J. González-Fraile, M.C. González-Garcia, Robust determination of the Higgs couplings: power to the data. Phys. Rev. D 87, 015022 (2013). arXiv:1211.4580 
49. T. Corbett, O.J.P. Éboli, J. González-Fraile, M.C. González-Garcia, Determining triple gauge boson couplings from Higgs data. Phys. Rev. Lett. 111, 011801 (2013). arXiv:1304.1151

50. Particle Data Group Collaboration, K.A. Olive et al., Review of particle physics. Chin. Phys. C 38, 090001 (2014)

51. ATLAS, CMS Collaboration, G. Aad et al., Combined measurement of the Higgs boson mass in $p p$ collisions at $\sqrt{s}=7$ and $8 \mathrm{TeV}$ with the ATLAS and CMS experiments. Phys. Rev. Lett. 114, 191803 (2015). arXiv: 1503.07589

52. M.E. Peskin, T. Takeuchi, A new constraint on a strongly interacting Higgs sector. Phys. Rev. Lett. 65, 964-967 (1990)

53. M.E. Peskin, T. Takeuchi, Estimation of oblique electroweak corrections. Phys. Rev. D 46, 381-409 (1992)

54. A. Pomarol, F. Riva, Towards the ultimate SM fit to close in on Higgs physics. JHEP 01, 151 (2014). arXiv:1308.2803

55. M. Ciuchini, E. Franco, S. Mishima, M. Pierini, L. Reina, L. Silvestrini, Update of the electroweak precision fit, interplay with Higgs-boson signal strengths and model-independent constraints on new physics, in International Conference on High Energy Physics 2014 (ICHEP 2014) Valencia, Spain, July 2-9, 2014 (2014). arXiv:1410.6940

56. SLD Electroweak Group, DELPHI, ALEPH, SLD, SLD Heavy Flavour Group, OPAL, LEP Electroweak Working Group, L3 Collaboration, S. Schael et al., Precision electroweak measurements on the $Z$ resonance. Phys. Rep. 427 (2006) 257-454. arXiv:hep-ex/0509008

57. CDF, D0 Collaboration, T.E.W. Group, 2012 Update of the combination of CDF and D0 results for the mass of the $\mathrm{W}$ boson. arXiv: 1204.0042

58. Tevatron Electroweak Working Group, CDF, DELPHI, SLD Electroweak and Heavy Flavour Groups, ALEPH, LEP Electroweak Working Group, SLD, OPAL, D0, L3 Collaboration, L.E.W. Group, Precision electroweak measurements and constraints on the standard model. arXiv:1012.2367

59. G. Isidori, A.V. Manohar, M. Trott, Probing the nature of the Higgslike boson via $h \rightarrow V \mathcal{F}$ decays. Phys. Lett. B 728, 131-135 (2014). arXiv:1305.0663

60. G. Isidori, M. Trott, Higgs form factors in associated production. JHEP 02, 082 (2014). arXiv: 1307.4051

61. M. Gonzalez-Alonso, A. Greljo, G. Isidori, D. Marzocca, Electroweak bounds on Higgs pseudo-observables and $h \rightarrow 4 \ell$ decays. Eur. Phys. J. C 75, 341 (2015). arXiv: 1504.04018

62. T. Corbett, O.J.P. Éboli, D. Goncalves, J. González-Fraile, T. Plehn, M. Rauch, The Higgs legacy of the LHC Run I. JHEP 08, 156 (2015). arXiv:1505.05516

63. E. Mass, V. Sanz, Limits on anomalous couplings of the Higgs boson to electroweak gauge bosons from LEP and the LHC. Phys. Rev. D 87(3), 033001 (2013). arXiv:1211.1320

64. S. Banerjee, S. Mukhopadhyay, B. Mukhopadhyaya, Higher dimensional operators and the LHC Higgs data: the role of modified kinematics. Phys. Rev. D 89(5), 053010 (2014). arXiv: 1308.4860

65. J. Ellis, V. Sanz, T. You, Complete Higgs sector constraints on dimension-6 operators. JHEP 07, 036 (2014). arXiv:1404.3667
66. J. Ellis, V. Sanz, T. You, The effective standard model after LHC Run I. JHEP 03, 157 (2015). arXiv: 1410.7703

67. R. Edezhath, Dimension-6 operator constraints from boosted VBF Higgs. arXiv: 1501.00992

68. R. Lafaye, T. Plehn, M. Rauch, D. Zerwas, M. Duhrssen, Measuring the Higgs sector. JHEP 08, 009 (2009). arXiv:0904.3866

69. M. Klute, R. Lafaye, T. Plehn, M. Rauch, D. Zerwas, Measuring Higgs couplings from LHC data. Phys. Rev. Lett. 109, 101801 (2012). arXiv:1205.2699

70. T. Plehn, M. Rauch, Higgs couplings after the discovery. Europhys. Lett. 100, 11002 (2012). arXiv: 1207.6108

71. M. Klute, R. Lafaye, T. Plehn, M. Rauch, D. Zerwas, Measuring Higgs Couplings at a Linear Collider. Europhys. Lett. 101, 51001 (2013). arXiv:1301.1322

72. D. Lopez-Val, T. Plehn, M. Rauch, Measuring extended Higgs sectors as a consistent free couplings model. JHEP 10, 134 (2013). arXiv: 1308.1979

73. T. Corbett, O.J.P. Eboli, D. Goncalves, J. Gonzalez-Fraile, T. Plehn, M. Rauch, The non-linear Higgs legacy of the LHC Run I. arXiv: 1511.08188

74. O.J.P. Eboli, J. Gonzalez-Fraile, M.C. Gonzalez-Garcia, Scrutinizing the $\mathrm{ZW}+\mathrm{W}$ - vertex at the large hadron collider at $7 \mathrm{TeV}$. Phys. Lett. B 692, 20-25 (2010). arXiv: 1006.3562

75. K. Hagiwara, R.D. Peccei, D. Zeppenfeld, K. Hikasa, Probing the weak boson sector in $e^{+} e^{-} \rightarrow W^{+} W^{-}$. Nucl. Phys. B 282, 253 (1987)

76. A. Butter, O.J.P. Éboli, J. González-Fraile, M.C. GonzálezGarcia, T. Plehn, The gauge-Higgs legacy of the LHC Run I. arXiv: 1604.03105

77. K. Hagiwara, R. Szalapski, D. Zeppenfeld, Anomalous Higgs boson production and decay. Phys. Lett. B 318, 155-162 (1993). arXiv:hep-ph/9308347

78. A. Falkowski, M. Gonzalez-Alonso, A. Greljo, D. Marzocca, Global constraints on anomalous triple gauge couplings in effective field theory approach. Phys. Rev. Lett. 116(1), 011801 (2016). arXiv: 1508.00581

79. A. Drozd, J. Ellis, J. Quevillon, T. You, Comparing EFT and exact one-loop analyses of non-degenerate stops. JHEP 06, 028 (2015). arXiv: 1504.02409

80. M. Gorbahn, J.M. No, V. Sanz, Benchmarks for Higgs effective theory: extended Higgs sectors. JHEP 10, 036 (2015). arXiv: 1502.07352

81. J. Brehmer, A. Freitas, D. Lopez-Val, T. Plehn, Pushing Higgs effective theory to its limits. Phys. Rev. D 93, 075014 (2016). arXiv: 1510.03443

82. A. Biektter, J. Brehmer, T. Plehn, Pushing Higgs effective theory over the edge. arXiv: 1602.05202

83. O.J.P. Éboli, M.C. Gonzalez-Garcia, Mapping the genuine bosonic quartic couplings. Phys. Rev. D 93, 093013 (2016). arXiv: 1604.03555

84. G.F. Giudice, C. Grojean, A. Pomarol, R. Rattazzi, The strongly-interacting light Higgs. JHEP 06, 045 (2007). arXiv:hep-ph/0703164 